\title{
GEOMETRIC STRUCTURES ON MANIFOLDS
}

\author{
by \\ Sam Saiki
}

B.S. in Mathematics, Cal Poly San Luis Obispo, 2010

M.S. in Mathematics, Cal Poly San Luis Obispo, 2010

Submitted to the Graduate Faculty of

the Kenneth P. Dietrich School of Arts and Sciences in partial fulfillment

of the requirements for the degree of Doctor of Philosophy in Mathematics

University of Pittsburgh

2017 


\section{UNIVERSITY OF PITTSBURGH \\ KENNETH P. DIETRICH SCHOOL OF ARTS AND SCIENCES}

This dissertation was presented

by

Sam Saiki

It was defended on

May 4th 2017

and approved by

J. DeBlois, Ph.D., Asst. Professor, Department of Mathematics, University of Pittsburgh

T. Hales, Ph.D., Professor, Department of Mathematics, University of Pittsburgh

B. McReynolds, Ph.D., Assoc. Professor, Department of Mathematics, Purdue University

G. Sparling, Ph.D., Assoc. Professor, Department of Mathematics, University of Pittsburgh

Dissertation Director: J. DeBlois, Ph.D., Asst. Professor, Department of Mathematics, University of Pittsburgh 


\title{
GEOMETRIC STRUCTURES ON MANIFOLDS
}

\author{
Sam Saiki, PhD \\ University of Pittsburgh, 2017
}

In this thesis I will introduce three questions that involve hyperbolic and projective structures on manifolds and present my progress toward their solution.

I prove that the Hilbert length spectrum (a natural generalization of the marked length spectrum) determines the projective structure on certain non compact properly convex orbifolds up to duality, generalizing a result of Daryl Cooper and Kelly Delp ("The marked length spectrum of a projective manifold or orbifold") in the compact case.

I develop software that computes the complex volume of a boundary unipotent representation of a 3-manifold's fundamental group into $\operatorname{PSL}(2, \mathbb{C})$ and $\operatorname{SL}(2, \mathbb{C})$. This extends the Ptolemy module software of Matthias Goerner and uses the theory of Stavros Garoufalidis, Dylan Thurston, and Christian Zickert found in "The complex volume of $\operatorname{SL}(n, \mathbb{C})$ representations of 3-manifolds". I apply my software to a census of Carlo Petronio and find non-trivial representations from non torus boundary manifolds. I also find numerical examples of Neumann's conjecture.

I develop theory and software which describes a deformation variety of projective structures on a fixed manifold. In particular, I compute the tangent space of the variety at the complete hyperbolic structure for the figure-eight knot complement. This is a philosophical continuation of Thurston's deformation variety in the hyperbolic setting, which is implemented in the 3-manifold software SnapPea. 


\section{TABLE OF CONTENTS}

1.0 INTRODUCTION $\ldots \ldots \ldots \ldots \ldots \ldots \ldots \ldots$

1.1 Rigidity of Convex Projective Structures on a Manifold or Orbifold . . . 2

1.2 Volumes of $\operatorname{SL}(n, \mathbb{C})$ and $\operatorname{PSL}(2, \mathbb{C})$ representations of 3-manifolds . . . . 4

1.3 Parameterize $\mathbb{R P}^{n}$ Structures on 3-Manifolds . . . . . . . . . . . . . . 7

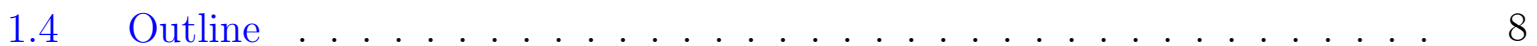

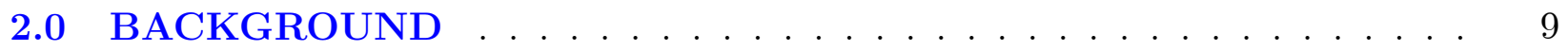

$2.1 \quad$ Hyperbolic Geometry . . . . . . . . . . . . . . . . . . . . . . . . 9

2.1.1 Hyperboloid Model . . . . . . . . . . . . . . . . . . 10

2.1.2 Upper Half Space Model . . . . . . . . . . . . . . . . . . 10

2.2 Manifolds with Boundary . . . . . . . . . . . . . . . . 11

2.3 Geometric Structures on Manifolds . . . . . . . . . . . . . . . 13

2.4 Example: Hyperbolic Structure on a 3 Holed Sphere . . . . . . . . . . . 14

2.4.1 $(G, X)$ Structure on $M \ldots \ldots \ldots 14$

2.4.2 Developing Map .................. 16

2.4.3 Geometric Structure as Discrete Faithful Representation . . . . 16

2.4.4 Geometric Structure as Quotient of $X$ by Discrete Subgroup of $G 16$

2.5 Interplay Between Notions of Geometric Structure . . . . . . . . . 17

2.5.1 $(G, X)$ Structure Induces a Representation and Development Map 18

2.5.2 Representations Induce Bundles with Flat Connection . . . . 19

2.5.3 Bundles with Flat Connection Induce Representations . . . . . 20

2.5.4 Principal G-Bundles with Flat Connection are Classified by $B G^{\delta} \quad 20$

2.5.5 Representations and Classifying Maps . . . . . . . . . . 21 


\subsection{HILBERT LENGTH SPECTRUM DETERMINES CONVEX PRO-}

JECTIVE STRUCTURE . . . . . . . . . . . . . . . . . . . . . 22

3.1 Convex Projective Orbifolds . . . . . . . . . . . . . . . . . . . . 23

3.1 .1 Examples . . . . . . . . . . . . . . . . . . . 23

3.1.2 Automorphisms of $\Omega \ldots \ldots \ldots \ldots \ldots \ldots$

3.1.3 Hilbert Metric and Translation Length . . . . . . . . . . . 26

3.1 .4 Ends . . . . . . . . . . . . . . . . . . . . . . . 27

3.2 Equality of Hilbert Translation Length is an Algebraic Condition . . . . 29

3.3 Zariski Density of $\Gamma \ldots \ldots \ldots \ldots \ldots \ldots$

3.3 .1 Strictly Convex . . . . . . . . . . . . . . . . . . . . . . 31

3.3 .2 Properly Convex . . . . . . . . . . . . . . . . . . . 31

3.4 Dual Projective Structures . . . . . . . . . . . . . . . . . . . . . 33

3.5 Main Theorem . . . . . . . . . . . . . . . . . . . . . . . . . . . . . 34

4.0 VOLUMES OF $\operatorname{SL}(2, \mathbb{C})$ AND PSL $(2, \mathbb{C})$ REPRESENTATIONS OF A

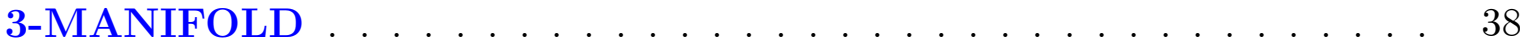

4.1 Hyperbolic 3 -manifolds . . . . . . . . . . . . . . . . . . . . . . . . . . . . 39

4.1.1 Boundary of Hyperbolic 3-Manifolds . . . . . . . . . . . . . 39

4.1.2 Boundary Parabolic Representations . . . . . . . . . . . 40

4.2 Ptolemy Variety . . . . . . . . . . . . . . . . . . . . . . . . . . . . 40

4.2.1 Construction of the Ptolemy Variety . . . . . . . . . . . . 41

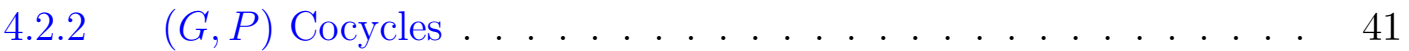

4.2.3 Ptolemy Variety Parameterizes $(G, P)$ Cocycles $\ldots \ldots \ldots . . .44$

4.2.4 Ptolemy Variety Parameterizes Boundary Parabolic Representations . . . . . . . . . . . . . . . . . . . . 44

4.2.5 PSL $(2, \mathbb{C})$ Representations via Obstruction Cocycles $\ldots \ldots .45$

4.3 Volume . . . . . . . . . . . . . . . . . . . . . . . . 45

4.3.1 Cheeger-Chern-Simons Invariant in the Compact Case . . . . 45

4.3 .2 CCS via Characteristic Classes . . . . . . . . . . . . 46

4.3.3 Extend CCS to Manifolds with Boundary . . . . . . . . . 47

4.3.4 Complex Volume via the Ptolemy Variety . . . . . . . . . 47 
$4.4 \quad$ Results . . . . . . . . . . . . . . . . . . . . . . . . . . 49

4.4.1 Frigerio, Martelli, and Petronio Census . . . . . . . . 50

4.4 .2 Invariant Trace Field . . . . . . . . . . . . . . . . 51

4.4 .3 Neumann's Conjecture . . . . . . . . . . . . . . . . . . 51

4.4.4 Future Direction . . . . . . . . . . . . . . . 52

$4.5 \quad$ Software Implementation . . . . . . . . . . . . . . . . . . . . 52

4.5.1 Complex Volumes Functions . . . . . . . . . . . . . 53

4.5 .2 Linear Combinations . . . . . . . . . . . . . . . . . 54

\subsection{PARAMETERIZING REAL PROJECTIVE STRUCTURES ON A}

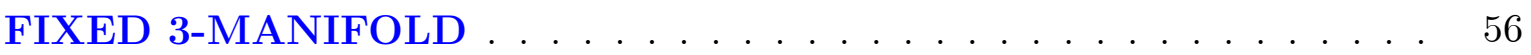

5.1 Introduction . . . . . . . . . . . . . . . . 56

$5.1 .1 \quad$ Strategy . . . . . . . . . . . . . . . . . 57

5.1.2 Motivation for Decorating with Point Hyperplane Pairs . . . . 57

$5.2 \quad$ Preliminaries . . . . . . . . . . . . . . . . . . 58

$5.2 .1 \quad$ Notation . . . . . . . . . . . . . . . . . . . . . . . . . 59

5.3 Tetrahedra of Partial Flags _ . . . . . . . . . . . . . . . . 59

$5.3 .1 \quad$ Partial flags . . . . . . . . . . . . . . . . . 60

5.3.2 Geometric Realization of Quadruples of Flags . . . . . . . . 60

5.3 .3 Action of $\mathrm{PGL}(4, \mathbb{R})$ on $P F \ldots \ldots \ldots \ldots$

5.3.4 Placing Elements of PGL $(4, \mathbb{R})$ in a Standard Position . . . . 62

$5.4 \quad$ Faces of Partial Flags and Face Identifications . . . . . . . . . . . . . 63

5.4.1 Face Parameter and Standard Position . . . . . . . . . . . 63

5.4 .2 Stabilizer of a Face . . . . . . . . . . . . . . . . 64

5.5 Projective Deformation Variety . . . . . . . . . . . . . . . 65

5.5 .1 Face Equations . . . . . . . . . . . . . . . 66

5.5.2 Edge Equations . . . . . . . . . . . . . . . . . . 66

5.5 .3 Gluing Equations . . . . . . . . . . . . . . . . 67

$5.6 \quad$ The Hyperbolic Case . . . . . . . . . . . . . . . . . . . . . . . . . . . . . 68

5.6 .1 Ideal Tetrahedron of Flags . . . . . . . . . . . . . 68

5.6 .2 Coordinates for Hyperbolic Tetrahedron . . . . . . . . . 68 
5.6.3 Coordinates for Regular Hyperbolic Tetrahedron . . . . . . . 68

$5.6 .4 \quad$ Alternative Inner Product . . . . . . . . . . . . . . . . 69

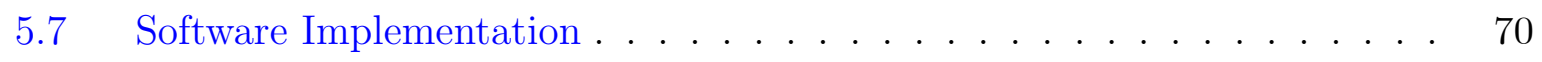

$5.7 .1 \quad$ Parameters . . . . . . . . . . . . . . . . 70

$5.7 .2 \quad$ Action of $\mathrm{PGL}(4, \mathbb{R}) \ldots \ldots \ldots \ldots \ldots$

5.7 .3 Edge Holonomy . . . . . . . . . . . . . . . . . . 70

5.8 Figure Eight Knot Complement . . . . . . . . . . . . . . . . . 71

$5.8 .1 \quad$ Face Gluing Matrices . . . . . . . . . . . . . . . . . . 72

$5.8 .2 \quad$ Face Pairing Equations . . . . . . . . . . . . . . 72

5.8 .3 Edge Holonomy Equations . . . . . . . . . . . . . . . 73

5.8 .4 Gluing Parameter Equations . . . . . . . . . . . . 75

5.8.5 Finding the Complete Hyperbolic Structure . . . . . . . . 75

5.8.6 Zariski Tangent Space at the Complete Hyperbolic Structure . . 76

5.9 Future Direction . . . . . . . . . . . . . . . . . . . . 76

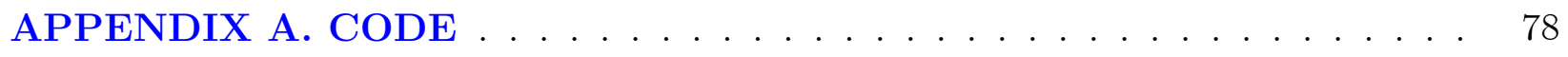

A.1 Fundamental Group Calculator . . . . . . . . . . . . . . 78

A.1.1 Fundamental Group of the Boundary . . . . . . . . . . . 79

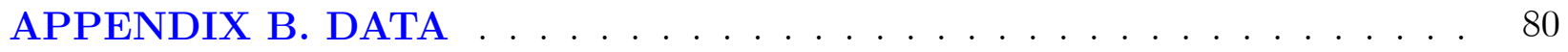

APPENDIX C. ALTERNATIVE HYPERBOLIC MODELS AND ISOMETRIES . . . . . . . . . . . . . . . . . . . . 83

C.1 The Hemisphere and Klein Model . . . . . . . . . . . . . . . . . . 83

C.2 Diffeomorphisms Between Models . . . . . . . . . . . . . . . . . 84

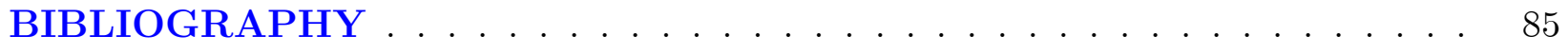




\section{LIST OF TABLES}

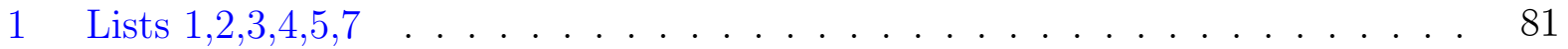

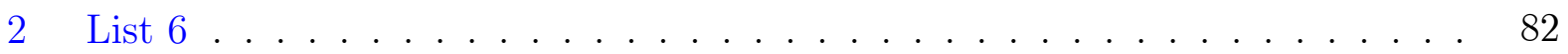




\section{LIST OF FIGURES}

1 Ideal Tetrahedron and Ordered Truncated Tetrahedron _. . . . . . . . . . . 12

2 Triangulation of 3 Holed Sphere . . . . . . . . . . . . . . . . . . . 14

3 Restriction of Identification to Edges . . . . . . . . . . . . . . . . . . 15

4 Developing Map . . . . . . . . . . . . . . . . . . . . 16

5 Holonomy Representation . . . . . . . . . . . . . . . . . . . 17

6 Hilbert Metric . . . . . . . . . . . . . . . . . . . . . . 27

7 Ptolemy Variety Decoration . . . . . . . . . . . . . . . . . . . . . . . 42

8 Labeled Cocycle . . . . . . . . . . . . . . . . . . . . . . . . . . . . 43

9 Ptolemy Assignment to $(\mathrm{G}, \mathrm{P})$ Cocycle . . . . . . . . . . . . . . . . . 44

10 Edge Holonomy . . . . . . . . . . . . . . . . . . . . . . . . . . 67

11 Triangulation of Figure 8 Knot Complement . . . . . . . . . . . 71

12 Variable Assignments . . . . . . . . . . . . . . . . . . . . . . . 73

13 Triangulation Around Edge in Various Models . . . . . . . . . . . . . . 77 


\section{LIST OF ALGORITHMS}

1 Complex Volumes of a List of Triangulations . . . . . . . . . . . . . . 54

2 edgeGluings algorithm . . . . . . . . . . . . . . . . 79 


\subsection{INTRODUCTION}

According to Klein's Erlangen program (1872), geometry is the study of the properties of a space invariant under the action of a group. In this vein, a geometry can be taken to be a space $X$ acted on transitively by a Lie group $G$. By a geometric structure on a manifold we mean an atlas of charts into $X$ such that the transition maps are elements of $G$. This is a powerful and flexible notion: in many interesting cases there are natural correspondences of geometric structures on a manifold $M$ and quotients of $X$ by discrete subgroups of $G$ acting freely and properly discontinuously, discrete and faithful representations of $\pi_{1}(M)$ into $G$, flat $G$-bundles over $M$, and maps from $M$ into the classifying space $B G^{\delta}$ of $G$ with discrete topology. We will describe these correspondences in the background chapter 2.

Geometric structures play an important role in modern mathematics. For instance, in dimension 2 Poincare proved the uniformization theorem: the universal cover of a Riemann surface is conformally equivalent to either the Riemann sphere, the Euclidean plane, or the hyperbolic plane, a theorem of widespread importance and use [51] W. Abikoff says "Of the major mathematical disciplines, few have not been enriched by the uniformization theorem or the methods developed for the study of the problem" [1]. In dimension 3 Perelman proved the geometrization conjecture: all oriented closed manifolds can cut along tori and spheres into pieces whose interior has one of eight geometric structures, all of which are subgeometries of projective geometry $\left(\mathbb{R} P^{3}, P G L(4, \mathbb{R})\right)[48]$ [49]. The largest and richest class of these structures is hyperbolic, where $X$ is the projectivization of the hyperboloid $x^{2}+y^{2}+z^{2}-w^{2}=1$ in $\mathbb{R}^{4}$ to $\mathbb{R} P^{3}$ and $G=P O(3,1)$ is the subgroup of $\operatorname{PGL}(4, \mathbb{R})$ stabilizing the projectivization (which turns out to be an open ball in $\mathbb{R} P^{3}$ ). Hyperbolic manifolds are then the quotient of $X$ by a discrete subgroup of $G$.

A natural way to generalize to projective geometry is to consider convex projective 
structures that come from the quotient of a convex domain $\Omega \subset \mathbb{R} P^{n}$ by a discrete subgroup $\Gamma \subset P G L(n+1)$ preserving it. Interestingly, there is a considerable difference between allowing $\Omega$ to have line segments in its boundary (properly convex) or not (strictly convex). In the first case $\Omega / \Gamma$ behaves similarly to a hyperbolic manifold, in the second, like an arbitrary symmetric space (see for instance chapter 0 of [14]).

In this thesis I will introduce three questions that involve hyperbolic and projective structures and present my progress toward their solution.

\subsection{RIGIDITY OF CONVEX PROJECTIVE STRUCTURES ON A MANIFOLD OR ORBIFOLD}

Equivalence is a basic problem in mathematics. In our context this is the question, how can I tell if two geometric structures on manifolds are isometric? Providing an actual isometry is often difficult, one approach is to define an invariant and ask if structures with equivalent invariants are isometric. A particularly striking theorem in the hyperbolic $\left(X=\mathbb{H}^{n}\right.$ and $G=$ $\operatorname{PSO}(n, 1))$ setting is Mostow-Prasad rigidity: finite volume hyperbolic $(n>3)$ manifolds are determined by their fundamental group (page 4 [44]). In other words, there is a unique conjugacy class of faithful representations $\pi_{1}(M) \rightarrow \operatorname{PSO}(n, 1)$. We will take this as a definition of rigidity of a representation, that there are no nonconjugate deformations. One can still ask if a hyperbolic 3 -manifold representation $\pi_{1}(M) \rightarrow \operatorname{PSO}(n, 1)$ is flexible in a larger Lie group such as $\operatorname{PGL}(n+1, \mathbb{R})$. In other words, is there a geometric notion that contains hyperbolic geometry within which the complete structure can be deformed?

In the case where $M$ contains a totally geodesic hypersurface, Johnson and Miller's bending construction provides deformations of the geometric representation (theorem 1 [35]). The corresponding geometric structures have been shown to be properly convex for manifolds

that are closed [40] and compact [42]. In the closed case Benoist showed that the structures are in fact strictly convex [8]. There is also some progress in the case that $M$ does not contain such a hypersurface. Cooper, Long, and Thistlethwaite used exact computation to find the projective deformation variety for 21 closed 3-manifolds with a complete hyperbolic structure 
[16]. Sam Ballas answered the question of flexibility in $\operatorname{PGL}(n+1)$ for the figure-eight knot complement by giving a deformation of the representation corresponding to the complete hyperbolic structure in [3]). In a subsequent paper he showed these representations actually corresponded to finite volume properly convex geometric structures on the figure-eight knot complement (theorem $1.1[2]$ ). Properly convex is necessary here, he also showed that there are no strictly convex deformations (theorem $1.1[3]$ ).

This leads to the question, is there an invariant that ensures rigidity for properly or strictly convex projective structures? One candidate is the marked length spectrum, an invariant of Riemannian manifolds that takes the infimum of loop length over each free homotopy class in the fundamental group. In the convex projective case, there is not necessarily a Riemanian metric. We do however have the Hilbert metric on any convex set, a complete Finsler metric that becomes the hyperbolic metric when the convex set is a ball. Thus we consider the Hilbert length spectrum which generalizes the marked length spectrum to properly convex orbifolds. Chapter 3 culminates in showing that the Hilbert length spectrum is such an invariant, generalizing work of Daryl Cooper and Kelly Delp in the compact setting [15]. A properly convex subset $\Omega \subset \mathbb{R} P^{n}$ is a convex subset of $\mathbb{R} P^{n}$ whose closure is disjoint from a hyperplane. A properly convex set that contains no line segments in the boundary is called strictly convex. A properly (strictly) convex projective orbifold $Q$ is the quotient $Q=\Omega / \Gamma$ of a properly (strictly) convex subset $\Omega$ of $\mathbb{R} P^{n}$ by a finitely generated discrete subgroup $\Gamma<P G L(n+1, \mathbb{R})$ preserving $\Omega$.

Theorem 3.5.5. (strictly convex rigidity). If $Q=\Omega / \Gamma$ is a finite volume strictly convex projective orbifold, the Hilbert length spectrum determines the projective structure up to duality.

In order to achieve a rigidity result in the properly convex setting, some kind of restriction on the orbifold's cusps is necessary, as described in section 3.1.4. Motivated by [14] and [5], I choose to restrict to orbifolds with generalized cusp ends, along with other natural hypothesis I collect under the adjective general (see section 3.1.4 for more details).

Theorem 3.5.6. (properly convex rigidity). If $Q=\Omega / \Gamma$ is general properly convex projective orbifold, the Hilbert length spectrum determines $\Gamma$ up to projective duality. 
These results continues a long lineage of marked length spectrum rigidity results. A. Katok et al conjectured in 1984 that the marked length spectrum determines the Riemannian metric on a compact manifold with non-positive curvature (conjecture 3.1 [11]). Since then progress has been made in various settings, the conjecture is true for closed surfaces (Theorem A [19]) and negatively curved locally symmetric manifolds [10].

To prove my main theorem in the properly convex setting, I must first show that the holonomy representation has Zariski dense image. This result appears in section 3.3.2.

Theorem 3.3.2. If $Q=\Omega / \Gamma$ is a properly convex orbifold with strongly irreducible isometry group $\Gamma$ whose ends are generalized cusps and the orbit of $\bar{\Gamma}$ contains an affine patch then the Zariski closure of $\Gamma$ is $S L(n, \mathbb{C})$.

The proof of this result imitates methods in [2] and hinges on the following heuristic: subgroups of $\operatorname{PGL}(n+1)$ with large orbit in $\mathbb{R} P^{n}$ must be large. This result is due to Benoist in the compact setting (theorem 3.6 [7]) and Crampon and Marquis in the strictly convex case (theorem $7.9[18]$ ).

\subsection{VOLUMES OF SL $(N, \mathbb{C})$ AND PSL $(2, \mathbb{C})$ REPRESENTATIONS OF 3-MANIFOLDS}

"The most basic and important invariant of a hyperbolic manifold is its volume" (Ratcliffe p.902 [52]). Because of Mostow-Prasad rigidity, in dimensions greater than 2, volume is not just a geometric, but a topological invariant. For instance, if the complement of a knot in the 3 sphere can be given a hyperbolic structure then its volume is a knot invariant. Such knots are plentiful, among the 1.7 million prime knots with up to 16 crossings, all but 32 have hyperbolic complements [34].

Let $M$ be a compact 3-manifold with boundary. In chapter 4, I study volume as an invariant of 3-manifold representations $\rho: \pi_{1}(M) \rightarrow \mathrm{SL}(2, \mathbb{C})$ that connects hyperbolic geometry and Chern-Simons theory. Before I give a definition of the volume of a representation, I will briefly indicate why representations are connected to something geometric like vol- 
ume. Given a hyperbolic structure on a closed 3-manifold $M$, there is an associated flat $\mathrm{SL}(2, \mathbb{C}$ ) bundle (see section 2.5.2). The uniqueness of horizontal lifts allows us to construct a discrete faithful representation $\pi_{1}(M) \rightarrow \mathrm{SL}(2, \mathbb{C})$ called the holonomy representation. Conversely, given a faithful representation $\pi_{1}(M) \rightarrow \mathrm{SL}(2, \mathbb{C})$ with discrete image $\Gamma$, then $M$ can be realized as the quotient of hyperbolic space $\mathbb{H}^{3}$ by $\Gamma$ and as such comes with a natural hyperbolic structure and notion of volume. Thus the study of discrete and faithful representations $\pi_{1}(M) \rightarrow \mathrm{SL}(2, \mathbb{C})$ is closely related to the study hyperbolic structures on $M$ and their volume.

Now for a formal definition. The Cheeger-Chern-Simons (CCS) invariant of a representation $\rho$ of $\pi_{1}(M)$ in $\operatorname{SL}(2, \mathbb{C})$ is given by the Chern-Simons integral

$$
\hat{c}(\rho)=\frac{1}{2} \int_{M} s^{*}\left(\operatorname{Tr}\left(A \wedge d A+\frac{2}{3} A \wedge A \wedge A\right)\right) \in \mathbb{C} / 4 \pi^{2} \mathbb{Z}
$$

where $A$ is the flat connection in the flat $\operatorname{SL}(n, \mathbb{C})$-bundle $E_{\rho}$ with holonomy $\rho$, and $s$ : $M \rightarrow E_{\rho}$ is a section of $E_{\rho}$ (equation $1.1[27]$ ). When the representation $\rho$ corresponds to a finite volume hyperbolic structure on $M$, the CCS invariant is called the complex volume and is related to the Cherns-Simons invariant and the hyperbolic volume of $M$ by $\hat{c}(\rho)=i(\operatorname{Vol}(M)+i C S(M))$ (equation $1.2[27])$.

In the case of finite volume $M$ with cusps, the holonomy representation is always boundaryunipotent ( $\rho$ sends boundary classes to unipotent elements), as discussed in section 4.1.2. Motivated by this, Garoufalidis, Thurston, and Zickert extend the notion of complex volume to boundary unipotent representations of cusped manifolds into $\operatorname{SL}(n, \mathbb{C})$ and $\operatorname{PSL}(n, \mathbb{C})[27]$. These authors also introduce the Ptolemy variety which parametrizes conjugacy classes of boundary unipotent representations. The key features of the Ptolemy variety is that both the variety and the complex volume of points in the variety are computable for simple manifolds. Goerner has implemented these features in the 3-manifold software SnapPy[20]: he provides functions that take in an ideally triangulated 3-manifold and output a set of complex volumes. The only cusped hyperbolic 3-manifolds that admit a finite volume hyperbolic structure have boundary a disjoint union of tori- this is reflected in SnapPy code, which only allows computations on such torus boundary manifolds. 
Requiring that a representation be boundary unipotent is a strong condition. A natural question is whether there are any such non trivial representations for manifolds with non torus boundary even though there is no geometric reason for them to exist. I answer this question affirmatively in chapter 4 .

Theorem 4.4.1. There exist non trivial boundary parabolic representations into $S L(2, \mathbb{C})$ from 3-manifolds with non torus boundary.

As SnapPy only deals with torus boundary manifolds, I used Python and Sage to independently implement the existing theory from [27] for any triangulated 3-manifold. I then searched for representations for 5128 manifolds in the Frigerio, Martelli, and Petronio census [23] of 3-manifolds with boundary of genus at least 2 and low complexity triangulations.

Remark 1.2.1. I found non-trivial representations for 49 out of the 5128 manifolds I studied. For those representations, 46 of the volumes I calculated were purely imaginary and 10 were purely real complex volume(note that some manifolds had more than one representation).

A surprising conjecture of Neumann (conjecture 1 [46]) combined with work of Garoufalidis, Thurston, and Zickert implies that the complex volume of boundary parabolic representations in $S L(n, \mathbb{C})$ or $P S L(n, \mathbb{C})$ are integral linear combinations of volumes of hyperbolic 3-manifolds (conjecture 1.16 [27]). Stephen Gilles and Peter Huston provided numerous numerical examples of Neumann's conjecture for representations of manifolds with a hyperbolic structure [28]. In the process they compiled a database of invariants of hyperbolic 3-manifolds including their volumes. It should be noted that not all of their examples are of the geometric representation of the manifold.

Once I computed the representations above, I used the Gilles, Huston database and a simple Pari function to search for volumes from the Petronio census which are integral linear combinations of hyperbolic 3-manifolds and obtained the following.

Theorem 4.4.3. There exist numerical examples of Neumann's conjecture for volumes of representations from 3-manifolds with non torus boundary. In particular, the volume of several representations is exactly the volume of a closed hyperbolic manifold.

This is particularly interesting since the manifolds don't admit a finite volume hyperbolic structure and the corresponding representations have no existing geometric interpretation. 


\subsection{PARAMETERIZE $\mathbb{R}^{N}$ STRUCTURES ON 3-MANIFOLDS}

Another natural problem in geometry is to find a space that parameterizes all geometric structures of a certain kind on a fixed manifold. For instance, W.P. Thurston showed that some hyperbolic structures on ideally triangulated 3-manifolds can be parameterized by a complex variety (dimension the number of cusps) defined by "gluing" equations (chapter 4 [54]).

Thurston's variety is constructed as follows. The abstract ideal triangulation is made up of tetrahedra with their vertices removed together with face identifications. Each ideal tetrahedra is embedded in $\mathbb{H}^{3}$ to acquire a geometric structure and the edges are labeled with complex variables that record the embedding. These "shape" variables are then constrained by a polynomial equation that guarantees the tetrahedra fit geometrically around all edges in the quotient of the triangulation. Solutions to these equations make up the "deformation variety". Thurston also introduced additional polynomial completeness equations. If a point in the deformation variety satisfies these completeness equations, then it corresponds to a complete hyperbolic structure on $M$ and a discrete and faithful representation into $\operatorname{Isom}\left(\mathbb{H}^{3}\right)$. By Mostow-Prasad rigidity, this structure is unique.

Thurston's deformation variety provides a computational tool for explicitly understanding how the complete hyperbolic structure can deform into incomplete structures. Even though the deformation space of holonomy representations of properly convex structures has been studied [17], no such tool exists for computing the deformations of the complete hyperbolic structure into convex projective structures. As noted in section 1.1 above, specific instances of deformations have been found, for instance by Sam Ballas for the figure-eight knot complement [3] [2].

The goal of chapter 5 is to generalize Thurston's gluing equations and provide a computational tool for parameterize a set of $\mathbb{R P}^{3}$ structures on the interior $M^{\circ}$ of a fixed 3-manifold $M$. My strategy is to turn an abstract triangulation of $M^{\circ}$ into a $\mathbb{R P}^{3}$ structure by embedding each tetrahedron into $\mathbb{R P}^{3}$ and decorating each vertex with a codimension 1 hyperplane containing it. These point hyperplane pairs generalize the hyperbolic situation, where the supporting hyperplanes are uniquely determined as tangent to the light cone. I define a 
projective deformation variety as solutions to equations which identify faces and ensure that there is a coherent structure around each edge. An interesting feature of $\mathbb{R P}^{3}$ structures in this variety is that the boundary holonomy necessarily preserves a point supporting hyperplane pair, a feature shared by strictly convex projective structures (section 5 of [14]).

I have automated the above approach in Mathematica and applied the software to the figure-eight knot complement. I obtain a complementary result to the deformations of Sam Ballas [3]. His curve of deformations have peripheral subgroups that preserve a partial flag and should be contained in the deformation variety I calculated: I compute the Zariski tangent space at the hyperbolic structure and find its dimension allows for such deformations.

Theorem 5.8.1. The tangent space to the gluing equation variety at the complete hyperbolic structure has dimension 24.

Although a complete explicit parameterization of this variety seems computationally unfeasible by the current methods, by introducing more equations that restrict the boundary holonomy, I hope to identify interesting subvarieties such as the incomplete hyperbolic structures and the Ballas deformations.

\subsection{OUTLINE}

General background material about geometric structures is found in chapter 2 followed by three fairly self contained chapters that mirror the sections in the introduction: Hilbert Length Spectrum Determines Convex Projective Structure 3, Volumes of $S L(n, \mathbb{C})$ and $\operatorname{PSL}(2, \mathbb{C})$ representations of a 3-Manifold 4, and Parameterizing Real Projective Structures on a Fixed 3-Manifold 5. 


\subsection{BACKGROUND}

The background material starts with a brief review of hyperbolic geometry in section 2.1. Section 2.2 discusses the manifolds we will put geometric structures on and section 2.3 the structures themselves. The interplay between the various viewpoints of geometric structures is introduced by example in 2.4 and theoretically in 2.5 . $0.0 \mathrm{pt}$ plus $1.0 \mathrm{pt}$

\subsection{HYPERBOLIC GEOMETRY}

Just as Euclidean space is the local model for a manifold, a geometric structure on a manifold is isometric to a model geometry. In this section we will introduce hyperbolic geometry as a model space. This particular geometry is the motivation for all three of the problems of this thesis, even though none of the settings are strictly hyperbolic. In chapter 3 and 5 the focus is on projective geometry, a generalization of hyperbolic geometry. Chapter 4 studies the complex volume of $\operatorname{PSL}(2, \mathbb{C})$ representations - the setting generalizes the important hyperbolic concepts of volume and geometric representation.

Hyperbolic space 3 space is denoted $\mathbb{H}^{3}$. It is the simply connected, complete 3 -dimensional Riemannian manifold with constant negative sectional curvature. For reference, in the following subsections I will give some common models of hyperbolic space and diffeomorphisms between them, following the notation of [13]. 


\subsubsection{Hyperboloid Model}

Define the Lorentz inner product $\left\langle\left(w_{1}, x_{1}, y_{1}, z_{1}\right),\left(w_{2}, x_{2}, y_{2}, z_{2}\right)\right\rangle_{l}=w_{1} w_{2}+x_{1} x_{2}+y_{1} y_{2}-z_{1} z_{2}$. Then the hyperboloid model of hyperbolic geometry is a connected component of the locus $\langle v, v\rangle=-1$ with the metric induced from the bilinear form. Explicitly:

$L=\left\{(w, x, y, z) \in \mathbb{R}^{4} \mid w^{2}+x^{2}+y^{2}-z^{2}=-1, z>0\right\}$ with metric $d s_{L}^{2}=d w^{2}+d x^{2}+d y^{2}-d z^{2}$.

The bilinear form restricts to a Riemannian metric on the tangent space to $L$ at $(w, x, y, z)$, which is the Lorentz-orthogonal complement to this vector. The indefinite orthogonal group $O(3,1)$ is the group of matrices that preserve the Lorentz inner product and thus the metric. By restricting to matrices that preserve the sign of the fourth component, we get the subgroup $O(3,1)$ that preserves the upper half of the hyperboloid $L$. The subgroup $S O^{+}(3,1)$ of determinant one matrices is called Lorentz group: it is the group of orientation preserving isometries of $L$. By the Klein perspective, the hyperbolic model of geometry is the pair $L, S O^{+}(3,1)$.

\subsubsection{Upper Half Space Model}

The upper half space model of hyperbolic space is

$$
H=\left\{(1, x, y, z) \in \mathbb{R}^{4} \mid z>0\right\} \text { with the metric } d s_{H}^{2}=\frac{d x^{2}+d y^{2}+d z^{2}}{z^{2}} .
$$

The boundary of $H$ is $\{(1, x, y, z) \mid z=0\}$ together with a point at $\infty$. Consider this to be $\mathbb{C}$ via $(x, y) \sim x+i y$ together with a point $\infty$ and call the result $\hat{\mathbb{C}}$. Then there is a natural action of $\operatorname{PSL}(2, \mathbb{C})$ on points $p \in \partial H=\hat{\mathbb{C}}$ by fractional linear maps:

$$
\left[\begin{array}{ll}
a & b \\
c & d
\end{array}\right] \cdot p=\frac{a p+b}{c p+d}
$$

This action of $\operatorname{PSL}(2, \mathbb{C})$ on $\partial H$ can be extended to $H$ as follows. Every element of $\operatorname{PSL}(2, \mathbb{C})$ is a composition of translations $p \mapsto p+\lambda$, dilations $p \mapsto \lambda p$, and reflections $p \mapsto-1 / p$. These can be extended to points $(1, x, y, z) \sim(p, z) \in H$ by $(p, z) \mapsto(p+$ 
$\lambda, z),(p, z) \mapsto(\lambda p,|\lambda| z)$, and $(p, z) \mapsto\left(\frac{-\bar{z}}{|z|^{2}+z^{2}}, \frac{z}{|p|^{2}+z^{2}}\right)$ respectively. By this construction $\operatorname{PSL}(2, \mathbb{C})$ becomes the group of orientation preserving isometries of $H$ and is isomorphic to $\mathrm{SO}^{+}(1,3)$. We can realize the isomorphism by defining the "spinor map" $s: \mathrm{SL}(2, \mathbb{C}) \rightarrow$ $\mathrm{SO}^{+}(1,3)$ which has kernel $\pm I$.

\subsection{MANIFOLDS WITH BOUNDARY}

Recall that a topological n-manifold is a second countable Hausdorff space that is locally homeomorphic to $\mathbb{R}^{n}$. The local homeomorphisms are called charts. A manifold with boundary has charts into the upper half space $\mathbb{R}_{+}^{n}:=\left\{\left(x_{1}, \ldots x_{n}\right) \mid x_{n} \geqslant 0\right\}$. A closed manifold is a compact manifold without boundary. In the following, I will mostly be considering geometric structures on non closed manifolds although much of the motivation and some of the techniques come from the closed case.

In chapter 3, I study noncompact manifolds and orbifolds. I will give a precise definition in 3.1 , but intuitively an orbifold is locally isomorphic to a quotient of $\mathbb{R}^{n}$ by a finite group action. It is important to understand what happens to a structure "near the edge" of a noncompact manifold. The starting point is the following definition: an end of a noncompact space $X$ is a function $e$ which assigns to each compact subset $K \subset X$ a nonempty component $e(K)$ of the complement $X \backslash K$, in such a way that $K \subset K^{\prime}$ implies $e\left(K^{\prime}\right) \subset e(K)$. In essence, the ends are the connected component of the open boundary of the space. For instance, a solid tetrahedron with the vertices removed (ideal tetrahedron) has 4 ends. In the context of this thesis, the noncompact manifolds often arise as the interior of a compact manifold with boundary. In this case the end components correspond to boundary components. A peripheral subgroup is a subgroup of the fundamental group $\pi_{1}(M)$ whose representative loops are homotopic to a connected component of the boundary.

Chapters 4 and 5 deal with triangulated 3-manifolds. An abstract triangulation of a manifold $M$ is a homeomorphism of $M$ with a 3 dimensional $\Delta$-complex (see section 2.1 of [33]. In other words, $M$ is built out of a collection of solid tetrahedra whose faces are identified with face pairings. An ordering on a tetrahedron is an ordering of the vertices, which we 
will denote $0,1,2,3$. A ordered triangulation is a triangulation of ordered tetrahedra where all the face pairings are order preserving(if edge $i, j$ is identified with edge $i^{\prime}, j^{\prime}$, then $i<j$ implies $\left.i^{\prime}<j^{\prime}\right)$. An ideal triangulation of a noncompact 3-manifold $M$ is a homeomorphism of $M$ with to the complement of the vertices of a 3 -dimensional $\Delta$-complex. An oriented triangulation of an oriented 3-manifold $M$ is an ideal triangulation such that the orientation of each tetrahedron agrees with the orientation of $M$. If an open neighborhood of each vertex in a tetrahedron is removed, the tetrahedron is called truncated. Such tetrahedra acquire an ordering from the tetrahedron ordering, as shown below. The edges of a truncated tetrahedron on the boundary of the removed open neighborhood are called boundary edges and the edges whose interior lies completely in the tetrahedron are called interior edges. In the figure below, all the long edges are interior and all the short edges are boundary. The faces with a 6 edge boundary are called interior faces and the faces with a 3 edge boundary are called boundary faces.
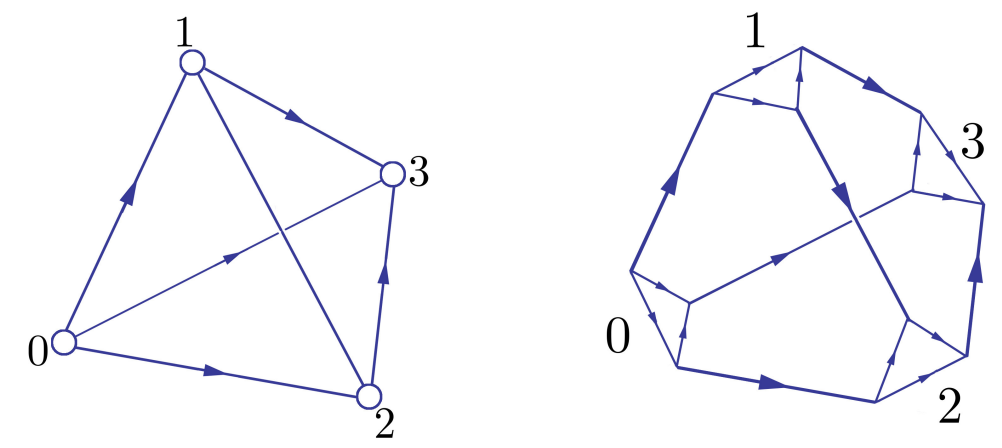

Figure 1: Ideal Tetrahedron and Ordered Truncated Tetrahedron

A truncated triangulation of a compact 3-manifold with boundary is a homeomorphism with the complement of open neighborhoods of vertices in a 3 dimensional $\Delta$-complex, that is, a triangulation where the tetrahedra are truncated. If an ideally triangulated manifold is the interior of a compact 3-manifold with boundary, truncating the ideal triangulation gives a truncated triangulation of the compact 3-manifold. 


\subsection{GEOMETRIC STRUCTURES ON MANIFOLDS}

As outlined in the Erlangen program [39], the Klein approach to geometry is to study the properties of a space invariant under the action of a Lie group. In this vein, a geometry can be taken to be a space $X$ acted on transitively by a Lie group $G$. The focus shifts from a local concept like a Riemannian metric to the group of isometries of that metric.

Let $G$ be a Lie group which acts on transitively on a manifold $X$. We can add geometric structure to a manifold $M$ by requiring that it is locally isomorphic to some geometry modeled by the action of $G$ on $X$. More precisely, following W. Goldman [31], a $(G, X)$ structure on a manifold is an atlas of charts into $X$ such that the transition maps are elements of $G$. If $M$ and $N$ are two $(G, X)$ manifolds, then a $f: M \rightarrow N$ is called a $(G, X)$ map if for any charts $\phi: M \supset U \rightarrow X$ and $\theta: N \supset V \rightarrow X$ the composition $\left.\theta^{-1} \circ f \circ \phi\right|_{\phi\left(U \cup f^{-1}(V)\right)}$ is the restriction of the action of an element of $G$ on $X$ to $\phi\left(U \cup f^{-1}(V)\right)$. The transition maps can be used to build a correspondence between $(G, X)$ structures and $G$-bundles with flat connection. This perspective of $(G, X)$ structures is useful, in part because such bundles are classified by homotopy classes of maps into the classifying space of $G$ with the discrete topology $B G^{\delta}$. The holonomy representation $\rho: \pi_{1}(M) \rightarrow G$ of the flat connection provides another object to study. This representation is equivariant with respect to the developing map Dev, a map from the universal cover $\widetilde{M}$ of $M$ to $X$ which extends the chart maps. Define $\Omega:=\operatorname{Dev}(\widetilde{M})$ and $\Gamma:=\rho\left(\pi_{1}(M)\right)$. In the case that Dev is a diffeomorphism onto its image, $\Gamma$ acts freely and properly discontinuously on $\Omega$ and we have that $M=\Omega / \Gamma$.

In many interesting cases, such when $M$ is a closed $n$-manifold, $X$ is hyperbolic $n$ space and $G$ the group of isometries of $X$, these viewpoints of geometric structures are actually equivalent up to natural equivalences, so that at your convenience or preference of global versus local or algebraic versus geometric versus topological, you can study quotients of $X$ acting by subgroups of $G$ acting freely and properly discontinuously, discrete and faithful representations of $\pi_{1}(M)$ into $G$ modulo conjugation, flat $G$-bundles over $M$, homotopy classes of maps from $M$ into $B G^{\delta}$, or simply $(G, X)$ structures.

The previous discussion indicates the flexibility and generality of geometric structures. As a particular example of usefulness, the geometrization conjecture solved by Perleman 
breaks a 3-manifold up into pieces endowed with one of eight geometric structures [48] [49].

The next two section provide an introduction to the interplay between the different notions of geometric structures which we will use for the remainder of this thesis. Section 2.4 gives a hands on briefing in the form of an example and pictures that introduces many of the key concepts, while section 2.5 provides more definitions and proofs and less pictures.

\subsection{EXAMPLE: HYPERBOLIC STRUCTURE ON A 3 HOLED SPHERE}

For the remainder of this section, $M$ is the three holed sphere. The goal is to put a hyperbolic $(G, X)$ structure on $M$, where $G=P S L(2, \mathbb{R})$ and $X=\mathbb{H}^{2}$. We can topologically triangulate $M$ with two ideal triangles (triangles with their vertices removed). There is a related manifold with boundary obtained by truncating the ideal triangles.
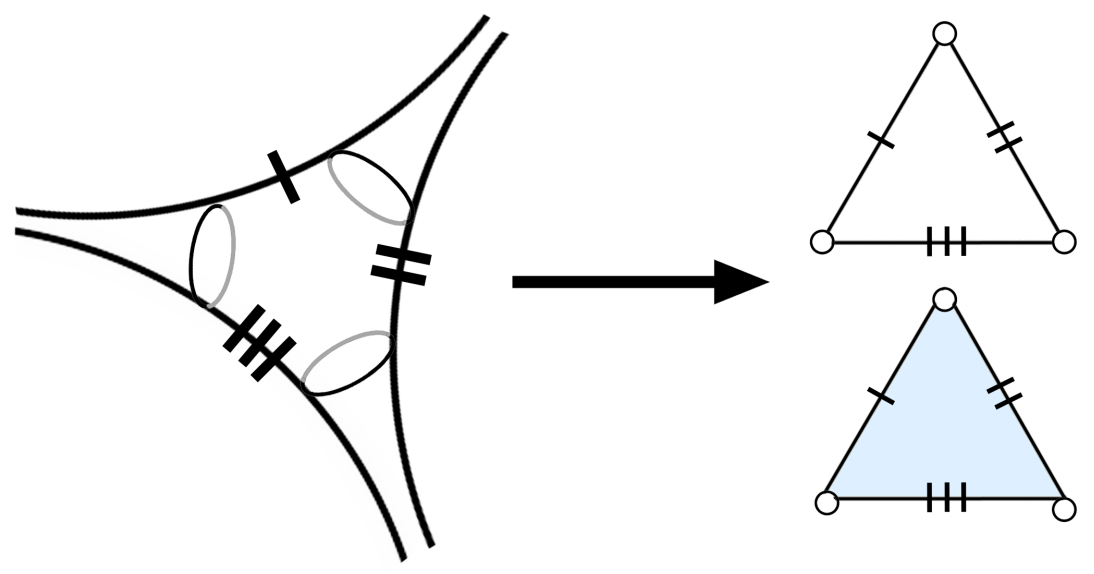

Figure 2: Triangulation of 3 Holed Sphere

\subsection{1 $(G, X)$ Structure on $M$}

To add a $(G, X)$ structure to $M$, form an atlas by embedding each triangle in $\mathbb{H}^{2}$ so that they share one edge. Choose isometries of $\mathbb{H}^{2}$ identifying the other two edges, so that the 
transition maps are restrictions of hyperbolic isometries. There are infinitely many ways to pair edges - if edge identifying isometries only have one fixed point, the geometric structure is complete.

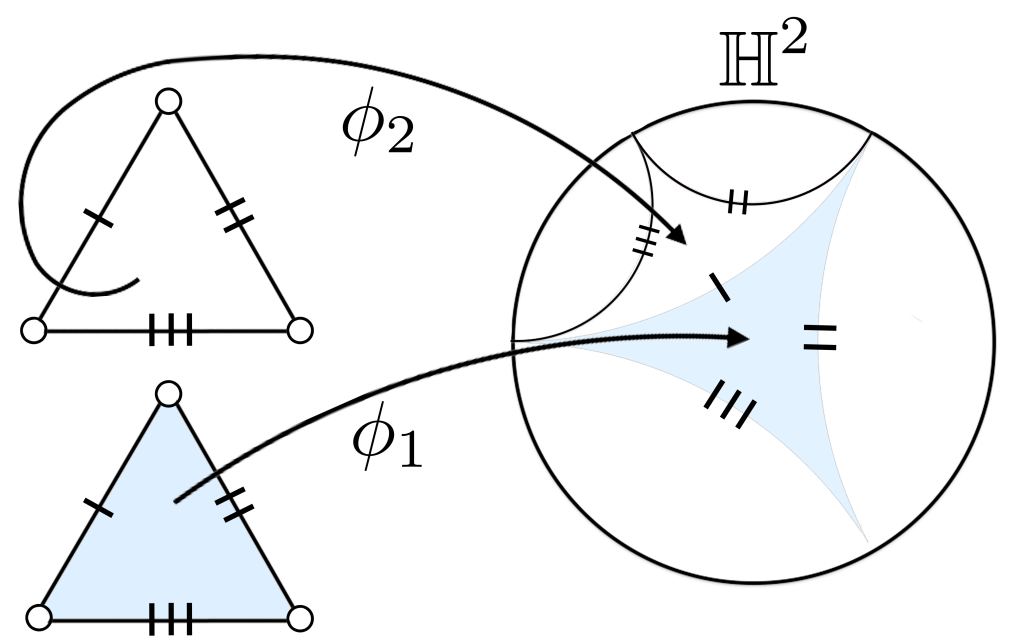

Depicted below is the identification of a edge by restricting the $\phi_{1}^{-1}$ to the double hashed edge $e$ and composing it with $\phi_{2}$. The restriction $\left.\phi_{1}^{-1} \circ \phi_{2}\right|_{e}$ is required to be a restriction of an isometry of $\mathbb{H}^{2}$.

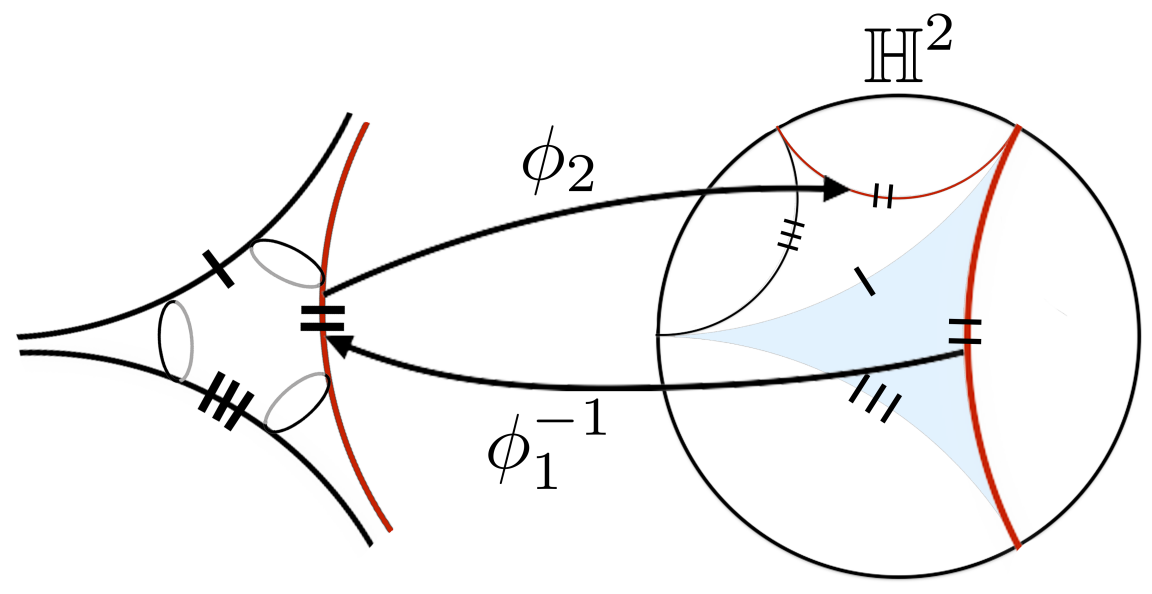

Figure 3: Restriction of Identification to Edges 


\subsubsection{Developing Map}

The chart maps can be extended to a "developing" map from the universal cover into $H^{2}$. Pictured in figure 4 is the developing map corresponding to the complete structure.

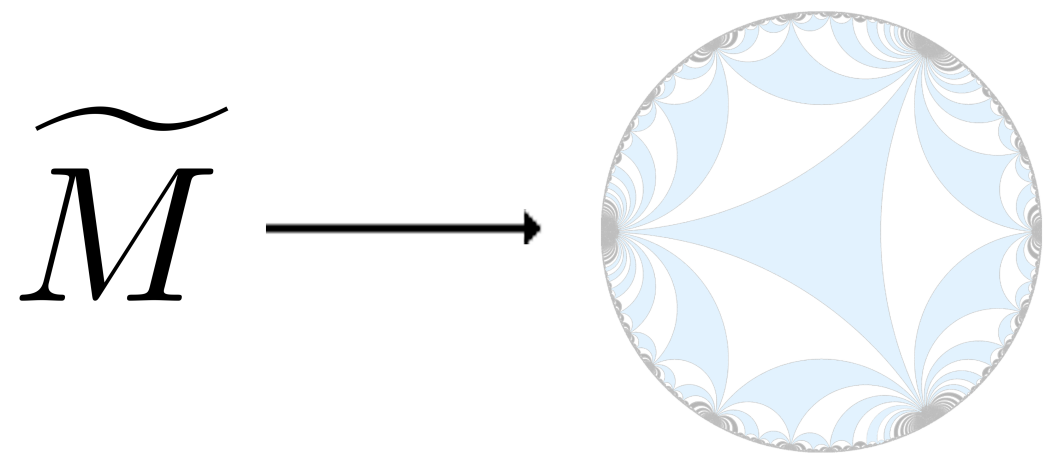

Figure 4: Developing Map

\subsubsection{Geometric Structure as Discrete Faithful Representation}

Given the developing map corresponding to the complete structure, we can define the "holonomy" representation hol : $\pi_{1}(M) \rightarrow \Gamma \subset I \operatorname{som}\left(\mathbb{H}^{2}\right)$. First find the dual skeleton of the triangulation, with vertices for each triangle and directed edges for each edge identification. Next find a maximal tree in this graph. The remaining edges in the dual skeleton represent fundamental group elements which get sent to the corresponding identification isometries by the holonomy representation. This representation is faithful and its image in $\operatorname{Isom}\left(\mathbb{H}^{2}\right)$ is discrete.

\subsubsection{Geometric Structure as Quotient of $X$ by Discrete Subgroup of $G$}

The image of the holonomy representation $\Gamma$ acts freely and properly discontinuously on $\mathbb{H}^{2}$ and the quotient of $\mathbb{H}^{2}$ by $\Gamma$ is diffeomorphic to $M$. These constructions fit into the following commutative diagram, as detailed in section 2.5.1. 

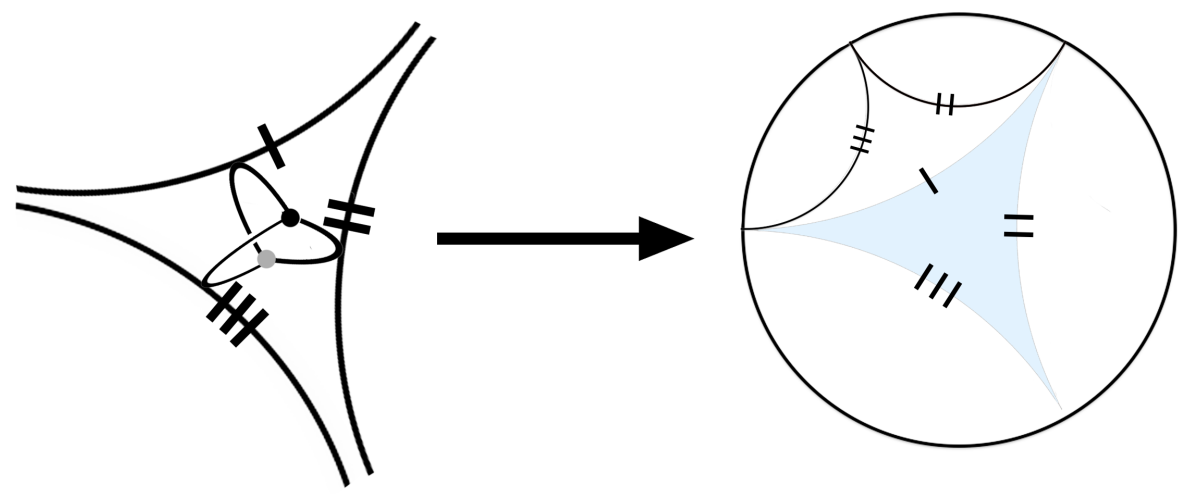

Figure 5: Holonomy Representation

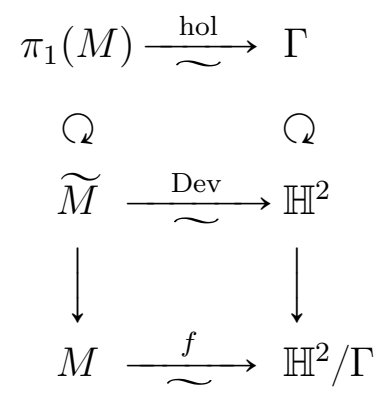

\subsection{INTERPLAY BETWEEN NOTIONS OF GEOMETRIC STRUCTURE}

This section indicates the connection between the definitions of geometric structure, often in the context of hyperbolic geometry, where the $(G, X)$ structures on a $M$ have $G=S L(2, \mathbb{C})$ and $X=\mathbb{H}^{3}$. As I have not found this material collected together at an expository level, I chose to make this section fairly detailed, though a knowledge of basic topology, cohomology, and Lie theory is assumed. 


\subsection{1 $(G, X)$ Structure Induces a Representation and Development Map}

Given a $(G, X)$ manifold $M$, the universal cover $p: \widetilde{M} \rightarrow M$ acquires a $(G, X)$ structure by pulling back along $p$. The following fundamental theorem is due to Ehresmann [22], we will use the version that appears as proposition 4.5 in [31] along with a full proof.

Theorem 2.5.1. (Development Theorem) Let $M$ be an $(G, X)$ manifold and $p: \widetilde{M} \rightarrow M$ its universal cover with deck transformations $\pi_{1}(M)$. Then there exists a pair $($ dev, $\rho)$ such that dev $: \tilde{M} \rightarrow X$ is a $(G, X)$ map and $\rho: \pi_{1}(M) \rightarrow G$ is a homomorphism such that, for each $\gamma \in \pi_{1}(M)$ the following diagram commutes

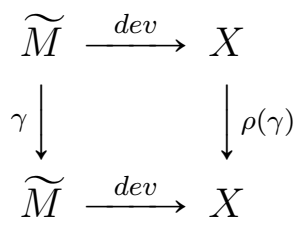

Furthermore, if $\left(\operatorname{dev}^{\prime}, \rho^{\prime}\right)$ is another such pair, there exists $g \in G$ such that dev $v^{\prime}=g \circ$ dev and $\rho^{\prime}(\gamma)=g \rho(\gamma) g^{-1}$ for each $\gamma \in \pi_{1}(M)$.

In the case that dev is a diffeomorphism onto $X$ we say that the $(G, X)$ structure is complete. Completeness when $X$ is simply connected is also equivalent to $\rho: \pi_{1}(M) \rightarrow \Gamma \subset G$ being an isomorphism onto discrete $\Gamma$ that acts freely and discontinuously on $X$ (for instance, see [31]). For complete structures we have the following diagram

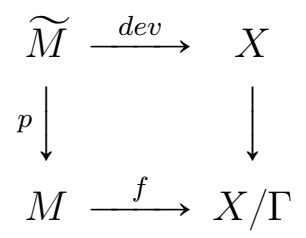

This demonstrates a useful tactic: the interchange the local concept of a $(G, X)$ structure on $M$ for the global notion of discrete subgroups of $G$ acting freely on $X$. In the hyperbolic case where $G=P S L(2, \mathbb{C})$ and $X=\mathbb{H}^{3}$, such discrete subgroups of $G$ are called Kleinian groups. 


\subsubsection{Representations Induce Bundles with Flat Connection}

The material in this section can be found in, for instance, 2.1.4 of [43]. Let $\rho: \pi_{1}(M) \rightarrow G$ be a representation. Let $\pi: \widetilde{M} \rightarrow M$ be the universal cover of $M$ and recall that $\pi_{1}(M)$ acts on $\widetilde{M}$ by deck transformations. Define an action of $\pi_{1}(M)$ on $\widetilde{M} \times G$ as follows: for $\gamma \in \pi_{1}(M)$ and $(m, g) \in \widetilde{M} \times G, \gamma(m, g)=(\gamma \cdot m, \rho(\gamma) \cdot g)$. Denote the quotient of $\widetilde{M} \times G$ by this action $\widetilde{M} \times{ }_{\rho} G$. Let $[m, g] \in \widetilde{M} \times{ }_{\rho} G$ be the equivalence class of $(m, g) \in \widetilde{M} \times G$. Now define $p_{1}: \widetilde{M} \times{ }_{\rho} G \rightarrow M$ by $p_{1}([m, g])=\pi(m)$ and a $G$ action on $\widetilde{M} \times{ }_{\rho} G$ by $[m, g] \cdot h \mapsto[m, g h]$ for $h \in G$. The next objective is to indicate that $p_{1}: \widetilde{M} \times_{\rho} G \rightarrow M$ is a principal $G$-bundle over $M$ (a fiber bundle with $G$ action on $P$ that preserves the fibers and acts freely and transitively on them).

Claim 2.5.2. The map $p_{1}$ is well defined and $p_{1}^{-1}(n) \simeq\{m\} \times G$ for $\pi(m)=n$.

Proof. Suppose $[m, g] \sim\left[m^{\prime}, g^{\prime}\right]$. Then $m^{\prime}=\gamma \cdot m$ which implies $\pi(m)=\pi\left(m^{\prime}\right)$, so $p_{1}$ is well defined. Now $p_{1}^{-1}(n)=\{[m, g] \mid \pi(m)=n\}$ by definition. I will show that $i:\{m\} \times G \rightarrow \widetilde{M} \times$ $G:(m, g) \mapsto[m, g]$ is a bijection onto $p^{-1}(n)$. To see $i$ is injective, assume $[m, g]=\left[m, g^{\prime}\right]$. Then $m=\gamma \cdot m$ which implies $\gamma$ is the identity since the deck transformation action is free. Thus $g^{\prime}=\rho$ (identity) $g=g$. Now check that $i$ is onto. Take $[q, g] \in p_{1}^{-1}(n)$, where $\pi(q)=n$. Then there exists $\gamma \in \pi_{1}(M)$ such that $\gamma \cdot q=m$. So $[q, g]=[\gamma \cdot q, \rho(\gamma) \cdot g]=[m, \rho(\gamma) \cdot g]$.

Local triviality of the bundle follows from the fact that $\pi$ is a covering map. Next we will see that conjugate representations induce the same bundle.

Claim 2.5.3. If $\rho$ and $\rho^{\prime}$ are conjugate representations, then $\widetilde{M} \times{ }_{\rho} G \simeq \widetilde{M} \times{ }_{\rho^{\prime}} G$.

Proof. Suppose $\rho^{\prime}(\gamma)=h \rho(\gamma) h^{-1}$ for $h \in G$. Then $[m, g] \rightarrow\left[m, h g h^{-1}\right]$ is a diffeomorphism.

Now that I have indicated how $p_{1}: \widetilde{M} \times_{\rho} G \rightarrow M$ is a principal $G$-bundle over $M$, I will introduce the flat connection on $G$ (a connection is an equivariant Lie algebra valued differential 1-form which defines a horizontal direction in the bundle, see for instance 10.1.2 [45]). Let $\omega_{M C}$ be the Maurer Cartan form on $G$ with Lie algebra $\mathfrak{g}$. Note that if $q: M \times G \rightarrow$ $G$ is the projection to the second factor, then $q^{*}\left(\omega_{M C}\right)$ is the trivial (flat) connection on the 
product bundle $M \times G \rightarrow G$. Similarly, let $\pi_{2}: \widetilde{M} \times{ }_{\rho} G \rightarrow G$ be the projection $[(m, g)] \mapsto g$. Then $\pi_{2}^{*}\left(\omega_{M C}\right)$ is the $\mathfrak{g}$ valued form on $\widetilde{M} \times{ }_{\rho} G$ that defines our connection. This connection is flat since

$$
d\left(\pi_{2}^{*} \omega_{M C}\right)=\pi^{*}\left(d \omega_{M C}\right)=\pi^{*}\left(-\omega_{M C} \wedge \omega_{M C}\right)=-\left(\pi^{*} \omega_{M C} \wedge \pi^{*} \omega_{M C}\right) .
$$

\subsubsection{Bundles with Flat Connection Induce Representations}

Let $P \rightarrow M$ be a principal $G$-bundle with flat connection. We can lift a curve in $M$ to $P$ horizontally to the connection. Since the connection is flat, given an initial point, the lift is unique. Let $[\alpha] \in \pi_{1}(M)$ and $\alpha$ be a representative curve based at $x$. Denote the lift of $\alpha$ to $P$ by $\bar{\alpha}$. Define the representation $\rho: \pi_{1}(M) \rightarrow G$ by $\rho(\alpha) \bar{\alpha}(1)=\bar{\alpha}(0)$. This is often known as the holonomy representation. Details can be found in, for instance, section 2.1.4 of [43]. In fact, we have the following theorem,

Theorem 2.5.4. (theorem 2.9, [43]) Let $M$ be a $C^{\infty}$ manifold and let $G$ be a Lie group. Then the correspondence which sends each principal flat G-bundle to its holonomy homomorphism induces a bijection

$\{$ isomorphism classes of prinicipal flat $G$-bundles over $M\} \simeq$ $\left\{\right.$ conjugacy classes of homomorphisms $\left.\rho: \pi_{1}(M) \rightarrow G\right\}$

\subsubsection{Principal $G$-Bundles with Flat Connection are Classified by $B G^{\delta}$}

Let $B G^{\delta}$ be the classifying space of $G$, when $G$ is equipped with the discrete topology. Recall that principal $G$-bundles with flat connection are classified by maps into $B G^{\delta}$ (theorem 2.1 [36]). That is, every principal $G$-bundle $P \rightarrow M$ is the pullback of the universal bundle $E G^{\delta} \rightarrow B G^{\delta}$ with flat connection. Conversely, if $f: M \rightarrow B G^{\delta}$ is a smooth map, then pulling back by $f$ gives a flat principal $G$-bundle over $M$. 


\subsubsection{Representations and Classifying Maps}

If $f: M \rightarrow B G^{\delta}$, then there is a induced map on fundamental groups $f_{*}: \pi_{1}(M) \rightarrow$ $\pi_{1}\left(B G^{\delta}\right)$. Since $\pi_{1}\left(B G^{\delta}\right)=G^{\delta}$, this is saying that classifying maps induce representations. The next objective is to show that for a 3-manifold $M$, a representation $\pi_{1}(M) \rightarrow G$ induces a classifying map $M \rightarrow B G^{\delta}$. In other words, that the map on fundamental groups can be upgraded to a map on spaces.

Claim 2.5.5. Let $M$ be a closed 3-manifold. A map $\rho: \pi_{1}\left(M, m_{0}\right) \rightarrow \pi_{1}\left(B G^{\delta}, b\right)$ induces a continuous map $R: M \rightarrow B G^{\delta}$ such that $R_{*}=\rho$.

Proof. Fix a maximal subtree $T$ of the 1 skeleton of the CW complex of $M$. For any one cell $e \notin T$, there exist $\gamma_{0}, \gamma_{1} \in T$ such that $\gamma_{0} e \gamma_{1} \in \pi_{1}(M, m)$. Choose a representation $\lambda:[0,1] \rightarrow B G$ of $\rho\left[\gamma_{0} e \gamma_{1}\right]$. Then for any point $e(t)$, define $R(e(t))=\lambda(t)$ and set $R(T)=b$. So $R$ is defined on the 1 skeleton of $M$, call this function $R^{1}$ and note that it is continuous and satisfies $R_{*}=\rho$ by definition.

Now we will define $R$ on 2-cells. For any 2-cell $f_{2}: D^{2} \rightarrow M$, take $B: I \rightarrow \partial D^{2}$ to be a path around the boundary and $\alpha: \partial D^{2} \rightarrow B G^{\delta}$ be the composition of $f$ and $R^{1}$. Now $[0,1] \stackrel{B}{\rightarrow} \partial D^{2} \stackrel{f_{2}}{\rightarrow} M$ is a loop homotopic to the identity in $\pi_{1}(M)$ and thus $[0,1] \stackrel{B}{\rightarrow} \partial D^{2} \stackrel{\alpha}{\rightarrow} B G^{\delta}$ is homotopic to the identity in $\left.\pi_{(} B G^{\delta}\right)$. Thus $\rho\left[R^{1} \circ f_{2} \circ B\right]$ is homotopic to the identity in $B G^{\delta}$, call the homotopy $H_{2}$. Specify some contraction $h$ of $D^{2}$, then set $R^{2}(f(h(s, t)))=H_{2}(s, t)$.

Now take a 3-cell $f_{3}: D^{3} \rightarrow M$. Since $G$ is discrete, $\pi_{2}\left(B G^{\delta}\right)$ is trivial and thus the

composition $\delta D^{3} \stackrel{f_{3}}{\longrightarrow} M \stackrel{R^{2}}{\longrightarrow} B G^{\delta}$ is homotopic to the identity. Call this homotopy $H_{3}$. Then define $R^{3}(f(s, t, u))=H_{3}(s, t, u)$. As $M$ is 3-dimensional we have completely defined $R$. 


\subsection{HILBERT LENGTH SPECTRUM DETERMINES CONVEX PROJECTIVE STRUCTURE}

The goal of this chapter is to prove that an interesting class of noncompact properly convex structures on an orbifold are determined up to projective duality by their Hilbert length spectrum. This generalizes a result of Daryl Cooper and Kelly Delp in the compact setting [15]. The main theorem relies on two key facts, that the Hilbert length spectrum can be computed algebraically and that the image of $\pi_{1}$ under the holonomy representation has large Zariski closure. Once these results are established, the arguments used by Cooper and Delp to prove theorem 5.1 in [15] can be adapted with slight modification.

In section 3.1, I review convex projective structures which are the quotient of a properly or strictly convex domain $\Omega$ by a discrete group of isometries $\Gamma$ acting on it. Key results include showing that matrices in $S L_{ \pm}(n+1, \mathbb{R}):=\{M \in G L(n+1, \mathbb{R}) \mid \operatorname{det}(M)= \pm 1\}$ act on $\Omega$. In section 3.2, I define the Hilbert translation length and show that it can be computed algebraically. Subsequently, the equal translation variety is introduced as (roughly) pairs of matrices in $S L_{ \pm}(n+1, \mathbb{R})$ with the same translation length - we will use the following to prove the main theorem.

Corollary 3.2.6. The equal translation length variety is a proper subvariety of $S L_{ \pm}(n, \mathbb{R}) \times$ $S L_{ \pm}(n, \mathbb{R})$ that does not contain $S O(n, 1) \times S O(n, 1)$.

The Zarisksi closure of the isometry group $\Gamma$ of an orbifold is denoted $\overline{\text { Gamma }}$ and is examined in section 3.3. We show that for the orbifolds we are interested in, the group is dense in $\operatorname{SL}(n+1, \mathbb{R})$ or $S O(n, 1)$. The analogous result in the strictly convex setting was

proved by Mickaël Crampon and Ludovic Marquis, it appears as theorem 7.9 in [18]. In the properly convex setting, we prove the following theorem, where the adjective general collects 
some natural hypothesis as explained in section 3.1.4.

Theorem 3.3.2. If $Q=\Omega / \Gamma$ is a general properly convex orbifold then $\bar{\Gamma}=S L(n+1, \mathbb{R})$.

Section 3.4 contains definitions about dual projective structures and recalls that outer automorphisms of $\operatorname{PGL}(n+1, \mathbb{C})$ are generated by the duality map. Finally, in section 3.5 I demonstrate that if two convex orbifolds with the same Hilbert length spectrum have isometry groups dense in $\mathrm{SL}(n+1, \mathbb{R})$ or $S O(n, 1)$, then the orbifold's isometry groups are projectively equivalent.

Theorem 3.5.1. Suppose $Q_{1}=\Omega / \Gamma_{1}$ and $Q_{2}=\Omega / \Gamma_{2}$ are properly convex structures with the same Hilbert length spectrum and $\overline{\Gamma_{i}}$ is either $S O(n, 1)$ or $S L(n+1, \mathbb{R})$. Then $\Gamma_{1}$ and $\Gamma_{2}$ are projectively equivalent or dual.

By section 3.3, this applies to finite volume strictly convex orbifolds and properly convex orbifolds with generalized cusp ends and strongly irreducible isometry groups summarized in the following theorems.

Theorem 3.5.5. (strictly convex rigidity). If $Q=\Omega / \Gamma$ is a finite volume strictly convex projective orbifold, the Hilbert length spectrum determines the projective structure up to duality.

Theorem 3.5.6. (properly convex rigidity). If $Q=\Omega / \Gamma$ is general properly convex projective orbifold, the Hilbert length spectrum determines $\Gamma$ up to projective duality.

\subsection{CONVEX PROJECTIVE ORBIFOLDS}

An affine patch of $\mathbb{R} P^{n}$ is a subset obtained by deleting a codimension 1 hyperplane. A subset $\Omega$ of $\mathbb{R} P^{n}$ is called convex if its intersection with every projective line is connected. If furthermore topological closure $\bar{\Omega}$ of $\Omega$ is contained in an affine patch, then it is called properly convex. A point $p$ in $\delta \Omega:=\bar{\Omega} \backslash \Omega$ is strictly convex if $\delta \Omega$ does not contain any affine line segment through $p$. A properly convex set $\Omega$ is called strictly convex if it is strictly convex at every point in $\delta \Omega$. For instance, the Klein model of $\mathbb{H}^{n}$ in $\mathbb{R} P^{n}$ is strictly convex. 
A simplex in a properly convex set is properly convex but not strictly convex. Finally, a projective line is convex, but not properly convex.

A properly (strictly) convex projective orbifold $Q$ is the quotient $Q=\Omega / \Gamma$ of a properly (strictly) convex subset $\Omega$ of $\mathbb{R} P^{n}$ by a finitely generated discrete subgroup $\Gamma<P G L(n+1, \mathbb{R})$ preserving $\Omega$. If $\Gamma$ acts freely then $Q$ is a manifold. The group $\Gamma$ is called the isometry group of $Q$ or the orbifold fundamental group and denoted $\pi_{1}^{\text {orb }}(Q)$. If no finite index subgroup of $\Gamma$ preserves a proper subspace of $\mathbb{R} P^{n}$ then $\Gamma$ is called strongly irreducible.

A strictly (properly) convex structure on an orbifold $M$ is a triple $(f, \Gamma, \Omega)$ : a diffeomorphism $f$ of $M$ with a strictly (properly) convex orbifold $\Omega / \Gamma$, where $f$ is called a marking. We can lift $f$ to a developing map as described in section 2.5.1. This gives an identification of deck transformation of $\widetilde{M}$ with automorphisms of $\Omega$ : the holonomy representation hol which fits in the following commutative diagram.

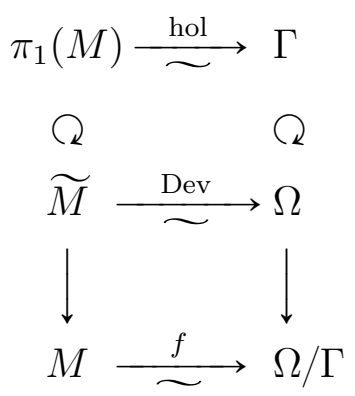

Two projective structures $\left(f, \Gamma_{1}, \Omega_{1}\right)$ and $\left(g, \Gamma_{2}, \Omega_{2}\right)$ on $M$ are equivalent if the markings are isotopic and differ by a projective map $A \in P G L(4, \mathbb{R})$, that is, the following diagram commutes: 


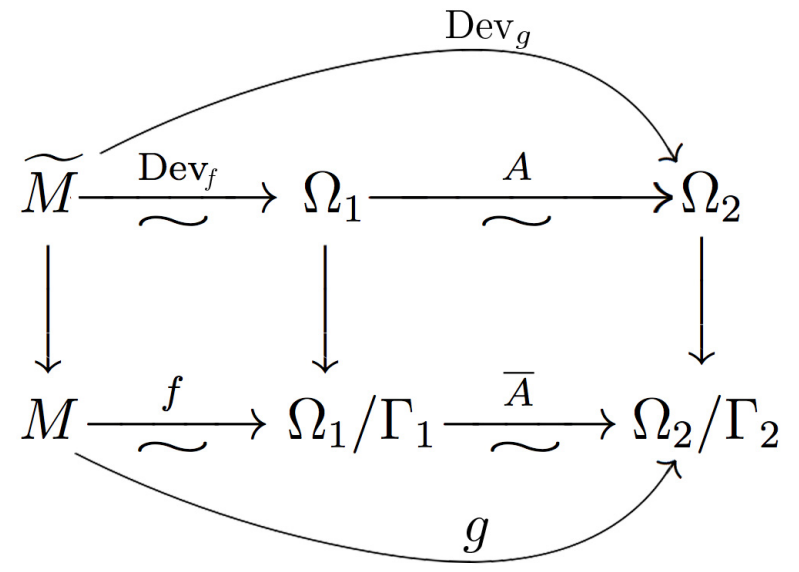

\subsubsection{Examples}

Hyperbolic orbifolds provide the simplest examples of strictly convex projective orbifolds, however, there are examples of strictly convex projective structures on orbifolds that do not admit a hyperbolic structure. See for instance, [37]. The quotient of the symmetric space $\mathrm{SL}(3, \mathbb{R}) / \mathrm{SO}(3)$ by $\mathrm{SL}(3, \mathbb{Z})$ is properly not strictly convex orbifold of finite volume (p. 3 $[14])$.

\subsubsection{Automorphisms of $\Omega$}

Let $S^{n}$ be the quotient of $\mathbb{R}^{n+1} \backslash\{0\}$ by the action of $\mathbb{R}^{+}$. We will denote the 2-1 cover of real projective space by the $n$-sphere as $\pi: S^{n} \rightarrow \mathbb{R} P^{n}$. The group of automorphisms of $S^{n} \subset \mathbb{R}^{n+1}$ is

$$
S L_{ \pm}(n+1, \mathbb{R}):=\{M \in G L(n+1, \mathbb{R}) \mid \operatorname{det}(M)= \pm 1\}
$$

Consider the map from the automorphisms of $S^{n}$ to the automorphisms of $\mathbb{R} P^{n}$ given by taking equivalence class:

$$
p: S L_{ \pm}(n+1, \mathbb{R}) \rightarrow P G L(n+1, \mathbb{R}): A \rightarrow[A]
$$

For the convenience of working with matrices, we sometimes would prefer to work in the cover $S L_{ \pm}(n+1, \mathbb{R})$. Let $\Omega$ be a convex domain and let $\operatorname{PGL}(\Omega)$ denote elements of $\operatorname{PGL}(n+1, \mathbb{R})$ that preserve $\Omega$. 
Claim 3.1.1. If $\Omega$ is properly convex, then $P G L(\Omega)$ is a subgroup of $S L_{ \pm}(n+1, \mathbb{R})$

Proof. We will provide a section of $p$ that is a homomorphism. Since $\Omega$ is contained in an affine chart, $\pi^{-1}(\Omega)$ consists of 2 disjoint copies of $\Omega$. Elements $M \in S L(\Omega)$ preserve or interchange the copies of $\Omega$. The section of $p$ defined by sending $[M]$ to its preimage that preserves the copies is thus a homomorphism.

We use $S L(\Omega)$ (respectively ) to denote elements of $S L_{ \pm}(n+1, \mathbb{R})$ that preserve a convex domain $\Omega$. There is a natural trichotomy of elements of $\mathrm{SL}(\Omega)$ analogous to the hyperbolic setting. Elements of $\operatorname{SL}(\Omega)$ are called elliptic if they fix a point in $\Omega$, parabolic if they act freely and every eigenvalue has value modulus 1 and hyperbolic otherwise [14].

\subsubsection{Hilbert Metric and Translation Length}

We can put a metric on a properly convex set $\Omega$ as follows. Recall that the toplogical closure of $\Omega$ lies in an affine patch, call it $A_{\Omega}$. Let $\|\cdot\|$ be a Euclidean metric on $A_{\Omega}$. Let $x, y \in \Omega$. Proper convexity implies that the affine line through $x$ and $y$ intersects $\delta \Omega$ in two points. Label these points so that they are ordered $x_{\infty}, x, y, y_{\infty}$. The Hilbert metric is the log of the cross ratio of these four points (note the cross ratio is projectively invariant):

$$
d_{H}:=\log \left(\frac{\left\|y-x_{\infty}\right\|}{\left\|x-x_{\infty}\right\|} \frac{\left\|x-y_{\infty}\right\|}{\left\|y-y_{\infty}\right\|}\right)
$$

The Hilbert metric is twice the hyperbolic metric (see section C.1) when $\Omega$ is the interior of an ellipsoid. Define Hilbert translation length as

$$
l: S L \Omega) \rightarrow \mathbb{R}: l(g)=\inf _{x \in \Omega} d_{H}(x, g x)
$$

Recall that for an orbifold $Q=\Omega / \Gamma, \Gamma$ and $\pi_{1}^{o r b}(Q)$ are identified by the holonomy represention $\rho: \pi_{1}^{\text {orb }}(Q) \rightarrow \Gamma$. The Hilbert length spectrum is the map from $\pi_{1}^{\text {orb }} \rightarrow \mathbb{R}$ defined by $L_{H}:=l \circ \rho$. 


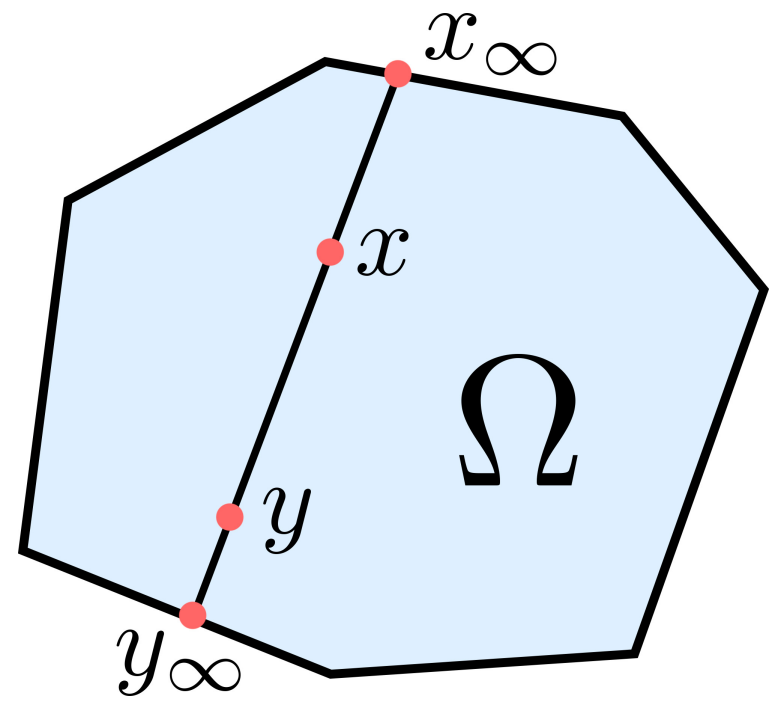

Figure 6: Hilbert Metric

\subsubsection{Ends}

The ends (defined in 2.2) of a strictly convex projective orbifold have algebraic constraints, for instance, the peripheral subgroup of the fundamental group corresponding to an end are virtually nilpotent and parabolic, as in the hyperbolic setting (section 5 [14]). The ends of properly convex projective orbifolds are less constrained: for instance, the peripheral subgroups can contain hyperbolics (p. 2 [17]). This extra freedom weakens the Hilbert length spectrum as an invariant: there exist non-dual, non-isometric properly convex structures on an orbifold. Since we are interested in studying orbifolds whose projective structures are determined up to projective dual by their Hilbert length spectrum, we need to place some additional restriction on the ends of the properly convex projective orbifold. One such restriction is made by Cooper, Long, and Tillman in [17], where they study the deformations of properly convex projective structures with generalized cusp ends.

Definition 3.1.2. (From [17]) A generalized cusp is a properly convex manifold $C$ homeomorphic to $\delta C \times[0, \infty)$ with compact, strictly convex boundary and with $\pi_{1} C$ virtually nilpotent. 
Sam Ballas, Daryl Cooper, and Arielle Leitner have classified generalized cusps in a work in preparation [5]. As mentioned in the introduction, in the properly convex setting, I will focus on orbifolds that have such generalized cusp ends and a few other hypothesis.

Let $Q=\Omega / \Gamma$ be a properly convex orbifold. Recall $\Gamma<\operatorname{PGL}(n+1)$ acts on $\mathbb{R} P^{n}$ and thus that Zariski closure of $\Gamma$ in $\operatorname{PGL}(n+1)$ (denoted $\bar{\Gamma}$ ) acts on $\mathbb{R} P^{n}$. We say $\Gamma$ has large orbit if the action of $\bar{\Gamma}$ on $\mathbb{R} P^{n}$ has an orbit that contains an affine patch.

Definition 3.1.3. A general properly convex orbifold is a properly convex orbifold with strongly irreducible isometry group $\Gamma$ whose ends are generalized cusps, none of which are strictly convex cusps. Furthermore, we require that $\Gamma$ has large orbit.

One direct result of the Ballas, Cooper, Leitner classification is that properly convex orbifolds with generalized cusp ends have isometry groups $\Gamma$ such that the orbit of $\bar{\Gamma}$ contains an affine patch in $\mathbb{R} P^{n}$, rendering the last hypothesis redundant. Based on discussion with Daryl Cooper about unpublished work with D. Long, and S. Tillman, I suspect the condition that $\Gamma$ be strongly irreducible is also unnecessarily strong and under mild hypothesis can be removed for all properly convex orbifolds with generalized cusp ends.

In the hyperbolic setting, the ends are controlled in part by the Margulis lemma, restated here as in $[6]$.

Theorem 3.1.4. (Theorem D.1.1 [6]) For all $n \in \mathbb{N}$ there exists $\epsilon_{n} \geqslant 0$ such that for any properly discontinuous subgroup $\Gamma<\operatorname{Isom}\left(\mathbb{H}^{n}\right)$ and for any $x \in \mathbb{H}^{n}$, the group $\Gamma_{\epsilon_{n}}(x)$ generated by the set

$$
F_{\epsilon_{n}}(x)=\left\{\gamma \in \Gamma: d(x, \gamma(x)) \leqslant \epsilon_{n}\right\}
$$

is virtually nilpotent.

As a corollary, for finite volume hyperbolic manifolds we have the "thick-thin" decomposition: every manifold is the disjoint union of a compact part and cusp ends (section D.2 [6]). Similarly, in the strictly convex setting we have:

Theorem 3.1.5. (Cooper, Long, Tillman Theorem .2 and .4 [14]) Finite volume strictly convex projective manifolds can be decomposed into a compact part and cusp ends.

There are properly convex manifolds which do not admit such a decomposition (see page 
2 of $[14])$.

\subsection{EQUALITY OF HILBERT TRANSLATION LENGTH IS AN ALGEBRAIC CONDITION}

Let $A$ be an automorphism of a properly convex domain $\Omega$. In this section I use results from "On Projective Manifolds and Cusps" [14] to show that the Hilbert translation length $l(A)$ can be computed as the log of real eigenvalues of $A$. Since eigenvalues are zeros of the characteristic polynomial, equality of translation length will define an algebraic variety in $\mathrm{SL}(n+1, \mathbb{R}) \times \operatorname{SL}(n+1, \mathbb{R})$. This is a key fact in the proof of the main theorem.

Theorem 3.2.1. (Prop 2.1 [14])Let $A \in S L(\Omega)$ for open properly convex $\Omega$. The Hilbert translation length of $A$ can be computed as the log of the ratio of the eigenvalues of maximum and minimum modulus: $l(A)=\log \left|\lambda_{\max } / \lambda_{\min }\right|$. Hyperbolic elements have positive translation length.

Recall $A$ is either elliptic, parabolic, or hyperbolic. If $A$ is elliptic, then by definition, $l(A)=0$. In the case that $A$ is parabolic, we have the following proposition:

Proposition 3.2.2. (Proposition 2.13 [14]) Suppose $\Omega$ is a properly convex domain and $A \in S L(\Omega, p)$ is parabolic then every eigenvalue has modulus 1 and the eigenvalue 1 has largest index, which is odd $\geqslant 3$.

Thus for parabolic $A, l(A)=\log |1| /|1|=0$. Finally, for hyperbolic elements we have the following.

Lemma 3.2.3. Suppose $\Omega$ is properly convex and $A \in S L(\Omega)$ is hyperbolic. Then $A$ has real eigenvalues of maximum and minimum modulus.

Proof. By (Prop. 2.6 [14]) $A$ has a real eigenvalue of maximum modulus. Similarly $A^{-1}$ has real eigenvalue $\lambda$ of maximum modulus. Thus $A$ has a real eigenvalue of minimum modulus $1 / \lambda$.

For $A \in \operatorname{GL}(n, \mathbb{R})$ with eigenvalues $\lambda_{1}, \ldots \lambda_{k}$, the numbers $\lambda_{i} / \lambda_{j}$ for $1 \leqslant i \neq j \leqslant k$ are 
called the eigenvalue ratios of $A$. Note eigenvalues ratios are well defined for elements of $\operatorname{PGL}(n, \mathbb{R})$ since for any real number $c$ the eigenvalue ratios of $c A$ are the same as $A$. In [15] the authors define the common root ratio polynomial and show that its roots are matrices which share an eigenvalue ratio, yielding the following result:

Proposition 3.2.4. (Proposition 3.10 Cooper, Delp [15]) For $n \geqslant 2$, let $A_{V} \subset S L_{ \pm}(n, \mathbb{R})^{2}$ be the set of pairs of matrices with a common ratio of eigenvalues. Then $A_{V}$ is a real algebraic proper subvariety.

Now I will show that pairs of matrices with the same Hilbert translation length share an eigenvalue ratio. To avoid a technicality with negative eigenvalues for now assume the real eigenvalues are positive. In the main proof the matrices in question will be squared to justify this assumption.

Definition 3.2.5. Let $\Omega$ be open and properly convex and let $T \subset S L_{ \pm}(n, \mathbb{R})^{2}$ be the set of pairs of parabolic or hyperbolic matrices in $\mathrm{SL}(\Omega)$ with the same translation length and positive real eigenvalues. Define the equal translation variety $T_{V}$ to be the Zariski closure of $T \cup(I, I)$.

Corollary 3.2.6. The equal translation variety $T_{V}$ is a proper subvariety of $S L_{ \pm}(n, \mathbb{R}) \times$ $S L_{ \pm}(n, \mathbb{R})$ that does not contain $S O(n, 1) \times S O(n, 1)$.

Proof. Suppose the Hilbert translation length is the same for $M, N \in \operatorname{SL}(n+1, \mathbb{R})$, that is, $l(M)=l(N)$. We will show that $M$ and $N$ share an eigenvalue ratio, so that the pairs of matrices with a shared translation length also share an eigenvalue ratio: in our notation, $T \subset A_{V}$, hence $T_{V} \subset A_{V}$.

Note $M$ and $N$ are either both parabolic with translation length 0 , or both hyperbolic with positive translation length by theorem 3.2.1. If they are both parabolic they share the $(\max / \mathrm{min})$ eigenvalue ratio $1 / 1$ by theorem 3.2.2. Suppose they are both hyperbolic. By lemma 3.2.3 the maximum and minimum modulus eigenvalues are realized by real (positive by assumption) eigenvalues, and we can remove the absolute values from the $\log$ ratio formula of Proposition 3.2.1: $\log \left(\lambda_{\mathrm{M} \max } / \lambda_{\mathrm{Mmin}}\right)=\log \left(\lambda_{\mathrm{Nmax}} / \lambda_{\mathrm{Nmin}}\right)$ implies $\lambda_{\mathrm{Mmax}} / \lambda_{\mathrm{M} \min }=\lambda_{\mathrm{Nmax}} / \lambda_{\mathrm{Nmin}}$ and $M$ and $N$ once again share an eigenvalue ratio. 
Since there are pairs of elements in $\mathrm{SO}(n, 1)$ which do not share any eigenvalue ratios, $P$ does not contain $\mathrm{SO}(n, 1) \times \mathrm{SO}(n, 1)$.

\subsection{ZARISKI DENSITY OF $\Gamma$}

For $Q=\Omega / \Gamma$ a strictly (properly) convex orbifold, denote the Zariski closure of $\Gamma$ by $\bar{\Gamma}$. In this section I show that for general properly convex orbifolds $\bar{\Gamma}=\mathrm{SL}(n+1, \mathbb{R})$. Crampon and Marquis have already proved the corresponding result for finite volume strictly convex orbifolds: $\bar{\Gamma}$ is either conjugate to $S O(n, 1)$ or $\operatorname{SL}(n+1, \mathbb{R})$ [18]. In the main theorem I will use the projective, complex version of these results: $P_{\mathbb{C}} \bar{\Gamma}$ is either $\operatorname{PSL}(n+1, \mathbb{C})=$ $P G L(n+1, \mathbb{C})$ or $P S O(n+1, \mathbb{C})$ combined with the classification of $\operatorname{Out}(P G L(n+1, \mathbb{C}))$ found in the next section.

\subsubsection{Strictly Convex}

Recall that in the finite volume strictly convex case, ends are parabolic. The density result in this case is due to Crampon and Marquis.

Theorem 3.3.1. (Theorem 7.9 Crampon, Marquis [18]) Let $Q=\Omega / \Gamma$ be a finite volume strictly convex orbifold. If $\Gamma$ contains a parabolic element, then $\bar{\Gamma}$ is either $S L(n+1, \mathbb{R})$ or conjugate to $S O(n, 1, \mathbb{R})$.

\subsubsection{Properly Convex}

Recall that a properly convex orbifold $Q=\Omega / \Gamma$ is called general if the ends are genuine (not strictly convex) generalized cusps, $\Gamma$ is strongly irreducible, and there is an orbit of $\bar{\Gamma}$ that contains an affine patch in $\mathbb{R} P^{n}$. In the work in progress "A Classification of Generalized Cusps on Properly Convex Projective n-Manifolds" [5], the last condition is actually shown to be unnecessary, since generalized cusps always have such an orbit.

Besides its use in the proof of the main theorem, the next result is of potential indepen- 
dent interest as a generalization of a theorem of Benoist in the compact case and the above theorem of Crampon and Marquis in the strictly convex case.

Theorem 3.3.2. If $Q=\Omega / \Gamma$ is a general properly convex orbifold then $\bar{\Gamma}=S L(n+1, \mathbb{R})$.

In order to prove this theorem I will introduce the notion of proximality and limit set and then employ arguments similar to those used to prove theorem 4.3 of [2]. Let $G$ be a subgroup of $\operatorname{SL}(n+1, \mathbb{R})$. An element $g \in G$ is proximal if it has a unique eigenvalue of largest modulus and the group $G$ is proximal if it contains a proximal element. For a strongly irreducible and proximal group $G \subset \operatorname{SL}(n+1, \mathbb{R})$, the limit set $\Lambda_{G}$ of $G$ is defined to be the closure of the set of attracting fixed point in $\mathbb{R} P^{n}$ of proximal elements of $G$. For our purposes, the important features are that $G$ acts transitively and invariantly on $\Lambda_{G}$ as proved in Theorem 2.3 of [32]. Let $\bar{\Gamma}^{\circ}$ be the connected component of the identity of $\bar{\Gamma}$. By [[32], Lemma 2.6], $\bar{\Gamma}^{\circ}$ is irreducible. It is straightforward to prove (see for instance [41] Lemma 2.1) that in this case $\bar{\Gamma}^{\circ}$ is semi-simple (a connected Lie group that does not contain non-trivial connected solvable normal subgroups).

Lemma 3.3.3. If $G<S L(n, \mathbb{R})$ acts irreducibly on $\mathbb{R}^{n}$, then $G$ does not leave invariant any subset $\Omega$ contained in a proper plane (codimension 1 subspace).

Proof. Suppose $G$ does leave invariant some such subset $\Omega$. We will show it also leaves a plane invariant. If $\Omega$ is a single point $p$ then $G$ leaves invariant on the line through $p$ and we're done.

Let $H$ be the subspace consisting of all linear combinations of elements of $\Omega$. The claim is that $G$ leaves invariant $H$. Since $H \subset P$, this will prove the claim. Take $x \in H$, then $x=\sum a_{i} x_{i}$ with $x_{i} \in \Omega$. Now $G x_{i} \in \Omega$ and thus $x$ is in $H$.

Lemma 3.3.4. Let $\Omega / \Gamma$ be a general properly convex orbifold. The limit set $\Lambda_{\bar{\Gamma}^{\circ}}$ is equal to $\mathbb{R} P^{n}$.

Proof. The orbit of $\bar{\Gamma}$ contains an affine patch by definition 3.1.3. Call this affine patch $U$. If $\Lambda_{\bar{\Gamma}^{\circ}} \cap U=\varnothing$, then $\Lambda_{\bar{\Gamma}^{\circ}}$ is contained in a codimension 1 subspace. This is impossible, since the irreducible $\Gamma^{\circ}$ would then leave invariant the space generated by $\Lambda_{\bar{\Gamma}^{\circ}}$, contradicting lemma 3.3.3. 
Let $z \in \Lambda_{\bar{\Gamma}^{\circ}} \cap U$. Since $\Lambda_{\bar{\Gamma}^{\circ}}$ is invariant under the action of $\Gamma, U \subseteq \Gamma \cdot z \subset \Lambda_{\bar{\Gamma}^{\circ}}$. Since $\Lambda_{\bar{\Gamma}^{\circ}}$ is closed, it is all of $\mathbb{R} P^{n}$.

Proof of theorem 3.3.2: We have shown $\bar{\Gamma}^{\circ}$ is semi simple, proximal and acts transitively on $\mathbb{R} P^{n}$. Thus it must be either $\operatorname{SL}(n+1, \mathbb{R})$ or $\operatorname{Sp}(n, \mathbb{R})$ by Lemma 3.9 of [7]. Another

result [Corollary 3.5] from the same paper rules out the second case for convex orbifolds, so $\bar{\Gamma}^{\circ}=\bar{\Gamma}=\operatorname{SL}(n+1, \mathbb{R})$.

\subsection{DUAL PROJECTIVE STRUCTURES}

In this section I review projectively dual structures and recall that outer automorphisms of $\operatorname{PGL}(n, \mathbb{C})$ are generated by the duality map.

Let $\Omega$ be a properly convex set. We can define the dual of $\Omega$ by a choice of inner product $\langle$,$\rangle on \mathbb{R}^{n+1}$ and preimage $\Omega_{+} \subset S^{n}$ of $\Omega$.

$$
\Omega^{*}:=\left\{x \in \mathbb{R}^{n+1} \mid\langle v, x\rangle>0 \text { for all } x \in \Omega_{+}\right\}
$$

The dual $\Omega^{*}$ is convex. If $A \in \operatorname{Aut}(\Omega)$ then $A^{t} \in A u t\left(\Omega^{*}\right)$. The dual map is the map

$$
d: P G L(n+1, \mathbb{R}) \rightarrow P G L(n+1, \mathbb{R}): A \rightarrow\left(A^{t}\right)^{-1} .
$$

Given a discrete group $\Gamma<\operatorname{Aut}(\Omega)$, the dual group is defined as $\Gamma^{*}:=d(\Gamma)$ and we say that $\Gamma$ and $\Gamma^{*}$ are projectively dual if $\Gamma^{*}=d^{\circ}(\Gamma)$ where $d^{\circ}$ is the dual map up to composition with an inner automorphism. The projective orbifold $Q=\Omega / \Gamma$ has dual orbifold $Q^{*}:=\Omega^{*} / d(\Gamma)$. If the dual map is a projective equivalence between $Q$ and $Q^{*}$, then we say $Q$ is self dual.

To prove our main result, I will show that two projective structures on an orbifold with the same marked spectrum differ by an automorphism of $\operatorname{PGL}(n+1, \mathbb{C})$. The following theorem from [15] implies that the corresponding isometry groups are projectively dual or equivalent.

Proposition 3.4.1. (Automorphisms of $P G L(n, \mathbb{C})$ Cor $4.6[15])$ For all $n \geqslant 2$ the group of holomorphic outer automorphisms $\operatorname{Out}(P G L(n, \mathbb{C})) \cong \mathbb{Z}_{2}$ is generated by the duality map. 
Proposition 3.4.2. Let $Q$ be a finite volume strictly convex projective orbifold. Then $Q$ is self-dual if and only if $Q$ is hyperbolic.

Proof. The following argument is the obvious adaptation of the proof of theorem 4.4 in [15].

The duality map takes a hyperbolic structure to a hyperbolic structure. This is because the ellipsoid $\Omega$ gets taken an ellipsoid $\Omega^{*}$ and the hyperbolic isometry group $P O(n, 1)$ is preserved by the duality map. By Mostow rigidity (Theorem $A^{\prime}$ page 4 of [44]) these structures are isometric and thus by proposition 2.9 of [15], projectively equivalent.

Now suppose $Q=\Omega / \Gamma$ is self-dual. By theorem 3.3.1, since $Q$ is strictly convex and finite volume, the Zariski closure of $\Gamma$ is either $\mathrm{SO}(n, 1)$ or $\mathrm{SL}(n+1, \mathbb{R})$. We will show $\Gamma$ is not Zariski dense in $\operatorname{SL}(n+1, \mathbb{R})$ by showing it is contained in the proper subvariety

$$
T:=\left\{A \in \mathrm{SL}(n+1, \mathbb{R}) \mid \operatorname{trace}(A)-\operatorname{trace}\left(A^{-1}\right)=0\right\}
$$

and conclude that $\bar{\Gamma}=\mathrm{SO}(n, 1)$.

By definition, there exists $P \in \operatorname{SL}(n+1, \mathbb{R})(\Omega)$ such that for all $A \in \Gamma$,

$$
d(A)=\left(A^{t}\right)^{-1}=P A P^{-1} .
$$

Thus $\Gamma<T$ and $\bar{\Gamma}=\mathrm{SO}(n, 1)$. Since strictly convex orbifolds are completely determined by their isometry group, we conclude $Q$ is hyperbolic.

\subsection{MAIN THEOREM}

Theorem 3.5.1. Suppose $Q_{1}=\Omega / \Gamma_{1}$ and $Q_{2}=\Omega / \Gamma_{2}$ are properly convex structures with the same Hilbert length spectrum and $\overline{\Gamma_{i}}$ is either $S O(n, 1)$ or $S L(n+1, \mathbb{R})$. Then $\Gamma_{1}$ and $\Gamma_{2}$ are projectively equivalent or dual. 
We will essentially follow the proof of Cooper and Delp. The two marked structures have holonomy representations $\pi_{1}^{\text {orb }}(Q) \rightarrow \Gamma_{i}$. Since $\Omega$ is properly convex we can lift the holonomy representations so that $\Gamma_{i} \subset \mathrm{SL}_{ \pm}(n+1, \mathbb{R})$. In the following we will work over $\mathbb{C}$ since the algebraic geometry becomes easier. Recall that the complexification of a real affine variety $V$ is the complex affine variety $V_{\mathbb{C}}$ obtained by taking the complex zeros of the real polynomial defining $V$.

Let $D$ be the Zariski closure over $\mathbb{C}$ of $\Gamma_{1} \times \Gamma_{2} \subset \mathrm{SL}_{ \pm}(n+1, \mathbb{C}) \times \mathrm{SL}_{ \pm}(n+1, \mathbb{C}), p_{i}$ be the projection to the $i^{\text {th }}$ factor, and define $G_{i}:=p_{i}(D)$.

$$
\begin{array}{ccc}
\mathrm{SL}_{ \pm}(n+1, \mathbb{C}) \times \mathrm{SL}_{ \pm}(n+1, \mathbb{C}) & \stackrel{p_{i}}{\longrightarrow} & \mathrm{SL}_{ \pm}(n+1, \mathbb{C}) \\
\cup & & \cup \\
\pi_{1}^{\text {orb }}(M) \stackrel{r_{1} \times r_{2}}{\longrightarrow} D:=\overline{\Gamma_{1} \times \Gamma_{2}} & \stackrel{p_{i}}{\longrightarrow} & G_{i}:=p_{i}(D) \supset \Gamma_{i}
\end{array}
$$

We projectivize using the natural projection $P: \mathrm{SL}_{ \pm}(n+1, \mathbb{C}) \rightarrow \operatorname{PGL}(n+1, \mathbb{C})$ and denote projection to the $i^{\text {th }}$ factor as $\pi_{i}$.

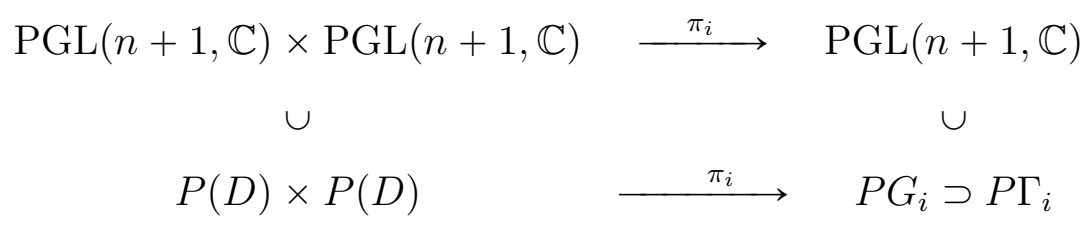

To prove the main result, I first show $P G_{i}=P\left(\overline{\Gamma_{i}}\right)$. Note that before complexification $\overline{\Gamma_{i}}=\operatorname{SL}(n+1, \mathbb{R})$ by hypothesis. Thus $P\left(\overline{\Gamma_{i}}\right)=P\left(\operatorname{SL}(n+1, \mathbb{R})_{\mathbb{C}}\right)=P(\operatorname{SL}(n+1, \mathbb{C}))=$ $\operatorname{PGL}(n+1, \mathbb{C})$. Next, I prove that $\pi_{i}: P(D) \times P(D) \rightarrow P G_{i}=\operatorname{PGL}(n+1, \mathbb{C})$ is an isomorphism, so that $\pi_{1} \circ \pi_{2}^{-1}$ is an automorphism of $\operatorname{PGL}(n+1, \mathbb{C})$. The theorem follows from the classification of automorphisms in section 3.4.1.

Lemma 3.5.2. $D$ is a proper subvariety of $S L_{ \pm}(n+1, \mathbb{C}) \times S L_{ \pm}(n+1, \mathbb{C})$ that does not contain $S O(n+1, \mathbb{C}) \times S O(n+1, \mathbb{C})$.

Proof. Because of Selberg's lemma [47], there is a torsion free subgroup of $\Gamma_{1}$ and $\Gamma_{2}$ of finite index, and thus finitely many orders of elliptic elements in $\Gamma_{1}$ and $\Gamma_{2}$. Let $k$ be the least common multiple of 2 and these orders and define $\tau: \operatorname{SL}(n+1, \mathbb{R}) \times \mathrm{SL}(n+1, \mathbb{R}) \rightarrow$ $\mathrm{SL}(n+1, \mathbb{R}) \times \mathrm{SL}(n+1, \mathbb{R})$ by $(M, N) \rightarrow\left(M^{k}, N^{k}\right)$. 
Recall $T_{V}$ is the variety of pairs of parabolic and hyperbolic isometries of $\Omega$ with the same translation length such that all real eigenvalues are positive. Since $\tau$ is a polynomial map, $\tau^{-1}\left(T_{V}\right)$ is an affine algebraic variety. To show $D:=\overline{\Gamma_{1} \times \Gamma_{2}}$ is a proper subvariety, I will check that $\Gamma_{1} \times \Gamma_{2} \subset \tau^{-1}\left(T_{V}\right)$.

Let $g \in \pi_{1}^{o r b}(Q)$. If $g$ is elliptic, then $\tau\left(r_{1}(g), r_{2}(g)\right)=(I, I)$ which is in $T_{V}$ by definition. By hypothesis $r_{1}(g)$ and $r_{2}(g)$ have the same translation length. Since translation length is calculated from eigenvalue ratios, $\tau\left(r_{1}(g)\right)$ and $\tau\left(r_{2}(g)\right)$ also have the same translation length. We defined $\tau$ to have a factor of 2 so that all the real eigenvalues of $\tau\left(r_{1}(g)\right)$ and $\tau\left(r_{2}(g)\right)$ are positive. Thus $\tau\left(r_{1}(g), r_{2}(g)\right) \in T_{V}$.

Note $\tau(\mathrm{SO}(n+1, \mathbb{C}) \times \mathrm{SO}(n+1, \mathbb{C}))=\mathrm{SO}(n+1, \mathbb{C}) \times \mathrm{SO}(n+1, \mathbb{C})$. Since $T_{V}$ does not contain $\mathrm{SO}(n+1, \mathbb{C}) \times \mathrm{SO}(n+1, \mathbb{C})$, neither does $D$.

Lemma 3.5.3. $\overline{\Gamma_{i}}=G_{i}$ and thus $P G_{i}=P\left(\overline{\Gamma_{i}}\right)=P G L(n+1, \mathbb{C})$.

Proof. This lemma follows from basic facts about algebraic groups. Since the Zariski closure of an algebraic subgroup is an algebraic subgroup, $D$ is a complex algebraic subgroup. As the image under an algebraic homomorphisms of a complex algebraic group is also a complex algebraic group, $G_{i}=p_{i}(D)$ is a complex algebraic subgroup of $\mathrm{SL}_{ \pm}(n+1, \mathbb{C})$. By definition $\Gamma_{i} \subset G_{i}$, so $\overline{\Gamma_{i}} \subset G_{i}$.

For the other inclusion note $\Gamma_{1} \times \Gamma_{2}$ is a subset of the complex algebraic group $p_{i}^{-1}\left(\overline{\Gamma_{i}}\right)$, so that $D:=\overline{\Gamma_{1} \times \Gamma_{2}} \subset p_{i}^{-1}\left(\overline{\Gamma_{i}}\right)$. Finally, $G_{i}=p_{i}(D) \subset p_{i}\left(p_{i}^{-1}\left(\overline{\Gamma_{i}}\right)\right)=\overline{\Gamma_{i}}$.

Lemma 3.5.4. $\pi_{i}: P(D) \times P(D) \rightarrow P G_{i}$ are isomorphisms.

Proof. $\pi_{i}$ is surjective onto $P G_{i}$ by definition. Assume to a contradiction that $\pi_{1}$ is not injective. Then we have the following diagram where the vertical arrows are isomorphisms:

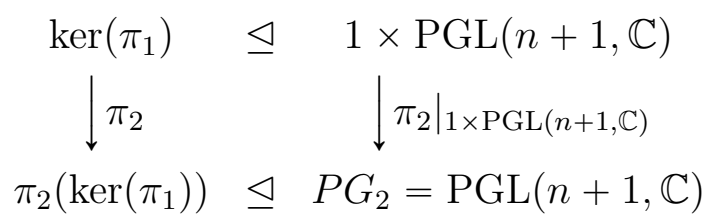

Thus $\pi_{2}\left(\operatorname{ker}\left(\pi_{1}\right)\right)$ is a nontrivial normal subgroup of $P G_{2}=\operatorname{PGL}(n+1, \mathbb{C})$. Lemma 4.7 of [15] says $\operatorname{PSO}(n+1, \mathbb{C}) \subset \pi_{2}\left(\operatorname{ker}\left(\pi_{1}\right)\right)$ so that $1 \times \operatorname{PSO}(n+1, \mathbb{C}) \subset \operatorname{ker}\left(\pi_{1}\right) \subset P(D) \times P(D)$. 
Thus $P \Gamma_{1} \times \mathrm{PSO}(n+1, \mathbb{C}) \subset P(D) \times P(D)$ which implies $P \overline{\Gamma_{1}} \times \mathrm{PSO}(n+1, \mathbb{C}) \subset P(D) \times P(D)$. Since $P \overline{\Gamma_{1}}=\operatorname{PGL}(n+1, \mathbb{C})$ and $\operatorname{PSO}(n+1, \mathbb{C}) \subset \operatorname{PGL}(n+1, \mathbb{C}), \operatorname{PSO}(n+1, \mathbb{C}) \times \operatorname{PSO}(n+$ $1, \mathbb{C}) \subset P(D) \times P(D)$, which contradicts lemma 3.5.2.

Now I can prove the main result, that $\Gamma_{1}$ and $\Gamma_{2}$ are projectively equivalent or dual. We have a isomorphism $\pi_{1} \circ \pi_{2}^{-1}: P G_{2} \rightarrow P G_{1}$ which by theorem 3.4.1 is either an inner automorphism or composition of the duality map and an inner automorphism. So $\Gamma_{1}$ and $\Gamma_{2}$ are related by conjugation in $\operatorname{PGL}(n+1, \mathbb{C})$, possibly composed with the duality map. In either case, since $\Gamma_{1}$ and $\Gamma_{2}$ are real, these automorphisms extend extends automorphisms of $\operatorname{PGL}(n+1, \mathbb{R})$. Finally, restricting to $\Gamma_{1} \subset G_{i}$ gives the desired result, concluding the proof of theorem 3.5.1.

From section 3.3 we saw that for strictly convex and general generalized orbifolds $\overline{\Gamma_{i}}$ is $\mathrm{SO}(n, 1)$ or $\mathrm{SL}(n+1, \mathbb{R})$. Thus, we have the following corollaries to the theorem 3.5.1.

Theorem 3.5.5. (strictly convex rigidity). If $Q=\Omega / \Gamma$ is a finite volume strictly convex projective orbifold, the Hilbert length spectrum determines the projective structure up to duality.

Theorem 3.5.6. (properly convex rigidity). If $Q=\Omega / \Gamma$ is general properly convex projective orbifold, the Hilbert length spectrum determines $\Gamma$ up to projective duality. 


\subsection{VOLUMES OF SL $(2, \mathbb{C})$ AND PSL $(2, \mathbb{C})$ REPRESENTATIONS OF A 3-MANIFOLD}

Let $M$ be a compact oriented 3-manifold with boundary such that its interior $M^{\circ}$ has an ordered ideal triangulation. As noted in section 2.2, $M$ has a corresponding ordered truncated triangulation. In this chapter I calculate volumes of boundary parabolic $\mathrm{SL}(2, \mathbb{C})$ and PSL $(2, \mathbb{C})$ representations of such manifolds $M$ using theory developed by Garoufalidis, Thurston, and Zickert [27]. The existing software (included in SnapPy [20] as the Ptolemy module) was developed for manifolds with torus boundaries - my implementation removes the boundary restriction. I obtain several interesting results by applying my software to the Frigerio, Martelli, Petronio census of 3-manifolds [23], including the following.

Theorem 4.4.1. There exist non trivial boundary parabolic representations into $S L(2, \mathbb{C})$ from 3-manifolds with non torus boundary.

I also developed code which searched for and found numerical examples of Neumann's conjecture. In particular:

Theorem 4.4.3. There exist representations from non torus boundary 3-manifolds whose volume is exactly that of a closed 3-manifold.

I will start by introducing some key ideas in the framework of hyperbolic geometry in section 4.1. Next I present an overview of the requisite material from [27]. Although the background results are valid for $\operatorname{PSL}(n, \mathbb{C})$ and $\operatorname{SL}(n, \mathbb{C})$, I will restrict to $n=2$ as that is the setting of my results. The Ptolemy variety is described in section 4.2 and an introduction to complex volume via the Cheeger-Chern-Simons invariant occurs in section 4.3. The main results occur in section 4.4 and an outline of the software implementation is in section 4.5. 


\subsection{HYPERBOLIC 3-MANIFOLDS}

The study of $\mathrm{SL}(n, \mathbb{C})$ representations of 3-manifolds is motivated and informed by hyperbolic geometry. In this section I will present ideas and definitions from hyperbolic 3-manifolds that will be generalized in subsequent sections.

The model geometry for hyperbolic 3 -manifolds is $\left(\mathbb{H}^{3}, \operatorname{PSL}(2, \mathbb{C})\right)$. Thus, as described in the background chapter 2, given a 3-manifold with a hyperbolic structure, we can extend the charts to a developing map and holonomy representation called the geometric representation.

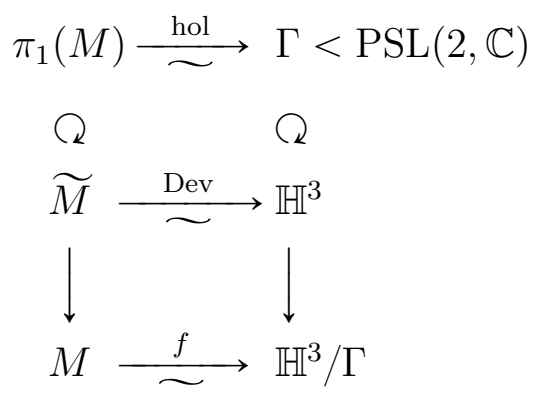

The main object of study in this chapter are representations from $\pi_{1}(M) \rightarrow P S L(2, \mathbb{C})$, where $M$ is a fixed 3-manifold. A hyperbolic structure on $M$ comes with a natural notion of volume:

Definition 4.1.1. $\mathrm{Vol}(\mathrm{M})$ is the integral of the pull back of the hyperbolic volume form over a fundamental domain of $M$.

\subsubsection{Boundary of Hyperbolic 3-Manifolds}

It follows from Margulis' lemma [38] (see for instance Corollary D.3.14 and Prop D.3.18 [6]) that any connected, oriented, complete, finite-volume hyperbolic 3-manifold $\mathrm{M}$ is diffeomorphic to the interior of a compact 3-manifold $N$ with (possibly empty) boundary. Each connected component $T$ of $\partial N$ (if it is non-empty) is diffeomorphic to a torus $\left(S^{1} \times S^{1}\right)$, and its inclusion in $N$ induces an injection at the level of fundamental group. 


\subsubsection{Boundary Parabolic Representations}

The moduli space of $\pi_{1}(M) \rightarrow P S L(2, \mathbb{C})$ representations is fairly complicated. One useful simplification is to only consider representations that are boundary parabolic(defined below), a feature of the geometric representation of a hyperbolic 3-manifold (this is another consequence of the Margulis lemma, see for instance theorem D.1.1 and D.1.13 in [6]). Recall from section 2.1.2 that elements of $\operatorname{PSL}(2, \mathbb{C})$ act on $\partial \mathbb{H}^{3}$.

Definition 4.1.2. A element of $\operatorname{PSL}(2, \mathbb{C})$ is parabolic if it fixes a unique point in $\partial \mathbb{H}^{3}$. A subgroup of $\operatorname{PSL}(2, \mathbb{C})$ is group parabolic if elements fix a common point in $\partial \mathbb{H}^{3}$

Let $P$ be the image in $\operatorname{PSL}(2, \mathbb{C})$ of upper triangular matrices with 1 's on the diagonal. Then group parabolic subgroups are conjugate to subgroups of $P$.

Definition 4.1.3. A representation $\pi_{1}(M) \rightarrow P S L(2, \mathbb{C})$ is called boundary parabolic if it sends peripheral subgroups to group parabolic subgroups. More generally, let $H$ be a subgroup of $G$. Then a representation into a group $G$ is called a $(G, H)$ representation if it sends peripheral subgroups into $H$.

\subsection{PTOLEMY VARIETY}

Garoufalidis, Thurston, and Zickert showed that the set of (conjugacy classes) of boundary parabolic representations of an ideally triangulated 3-manifold is parameterized by a variety they call the Ptolemy variety (Theorem 1.3 [27]). M. Goerner has implemented an algorithm that calculates the Ptolemy variety for $M$ with sufficiently simple triangulations. Using this implementation they have calculated the Ptolemy variety for all manifolds in the orientable cusped census with up to 9 simplices [30]. This section recalls the main results of [27]. I will first describe the construction of the Ptolemy variety and introduce the idea of $(G, P)$ cocycles. Next, I will indicate how the Ptolemy variety parameterizes $(G, P)$ cocycles and $(G, P)$ cocycles parameterize boundary parabolic representations. 


\subsubsection{Construction of the Ptolemy Variety}

This reprises section 5 of [27]. Let $M$ be a compact 3-manifold with boundary such that interior $M^{\circ}$ has ordered ideal triangulation $\mathcal{T}$. Let $\mathcal{T}_{t}$ be the associated ordered truncated triangulation of $M$. Note that $M$ and $M^{\circ}$ have the same fundamental group. The Ptolemy variety generalizes Thurston's deformation variety (see section 1.3). Recall that Thurston's variety is defined by labeling the edges of an triangulation with a complex number that records the geometric shape of each tetrahedron and then adding polynomial equations that ensure the geometric tetrahedra are glued together geometrically. The Ptolemy variety is defined in a similar fashion, although the edge decorations don't necessarily endow each tetrahedron with a geometric structure.

To construct the Ptolemy variety, first assign a nonzero complex number to each edge in the simplicial complex $\mathcal{T}$, as seen below on a particular tetrahedron. In other words, label the edges of each tetrahedron that makes up $M^{\circ}$ and then require that identified edges have the same labeling.

For each tetrahedron require that

$$
c_{03} c_{12}+c_{01} c_{23}=c_{02} c_{13} .
$$

The set of all such complex number assignments that satisfy equation 4.1 is called the Ptolemy variety. Thus for a triangulation with $m$ tetrahedron and $n$ quotient edges, points in the variety are solutions to a system of $m$ degree 2 homogeneous polynomials in $n$ variables.

\subsection{2 $(G, P)$ Cocycles}

To see that the Ptolemy variety parameterizes boundary parabolic representations of $M$, we need to introduce an intermediate set, that of $(G, P)$ cocycles. These cocycles are decorations of the truncated triangulation $\mathcal{T}_{t}$ of $M$ and will naturally correspond to boundary parabolic representations. Though much of the machinery developed is more general, for our purposes

specialize to $G=\mathrm{SL}(2, \mathbb{C})$ and $P$ the unipotent subgroup of upper triangular matrices with 1's on the diagonal. As described in section 9 of [27], given a triangulation of $M$, a $(G, P)$ cocycle is constructed by assigning an element of $\mathrm{SL}(2, \mathbb{C})$ to each edge of $\mathcal{T}_{t}$ where 


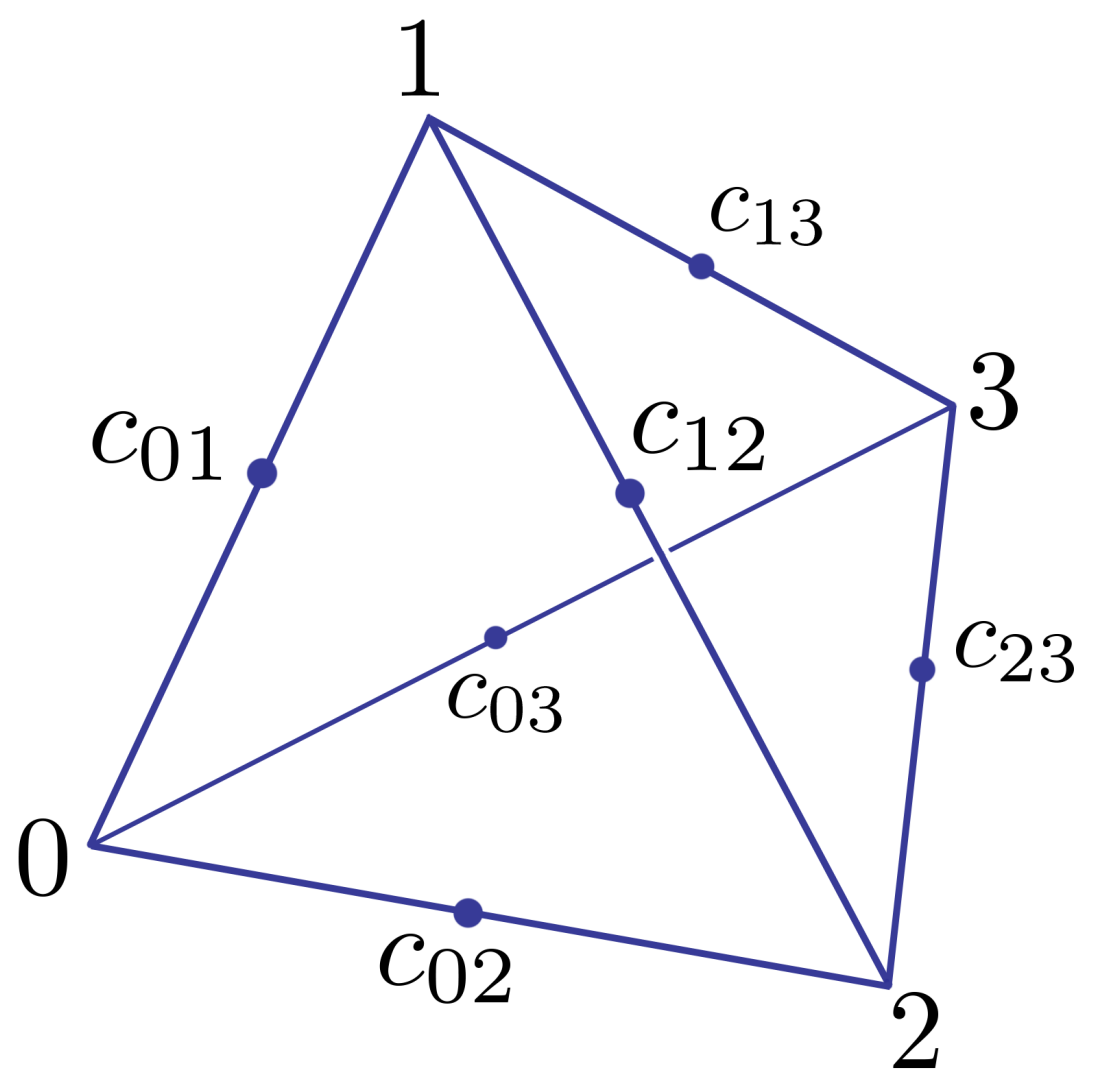

Figure 7: Ptolemy Variety Decoration

- Interior edges are decorated with elements of the form $\left(\begin{array}{cc}0 & -x^{-1} \\ x & 0\end{array}\right)$.

- Boundary edges are decorated with elements of the form $\left(\begin{array}{ll}1 & r \\ 0 & 1\end{array}\right)$.

The figure below shows a decorated truncated tetrahedron, where interior edges $(i, j)$ are decorated with $\alpha_{(i, j)} \in \mathrm{SL}(2, \mathbb{C})$ and boundary edges of vertex $k$ on the $(i, j)$ side are labeled with $\beta_{(i, j)}^{k}$. To avoid visual clutter in the figure, I have only labeled the edges around the boundary triangle associated to vertex 1 and the interior face opposite vertex 3 .

Furthermore, for each truncated tetrahedron require that the product (respecting orientation) around each face is the identity $I \in \mathrm{SL}(2, \mathbb{C})$. This gives a 6 term relation for each interior face and a 3 term relation for each boundary face. For instance, in figure 4.2.2 


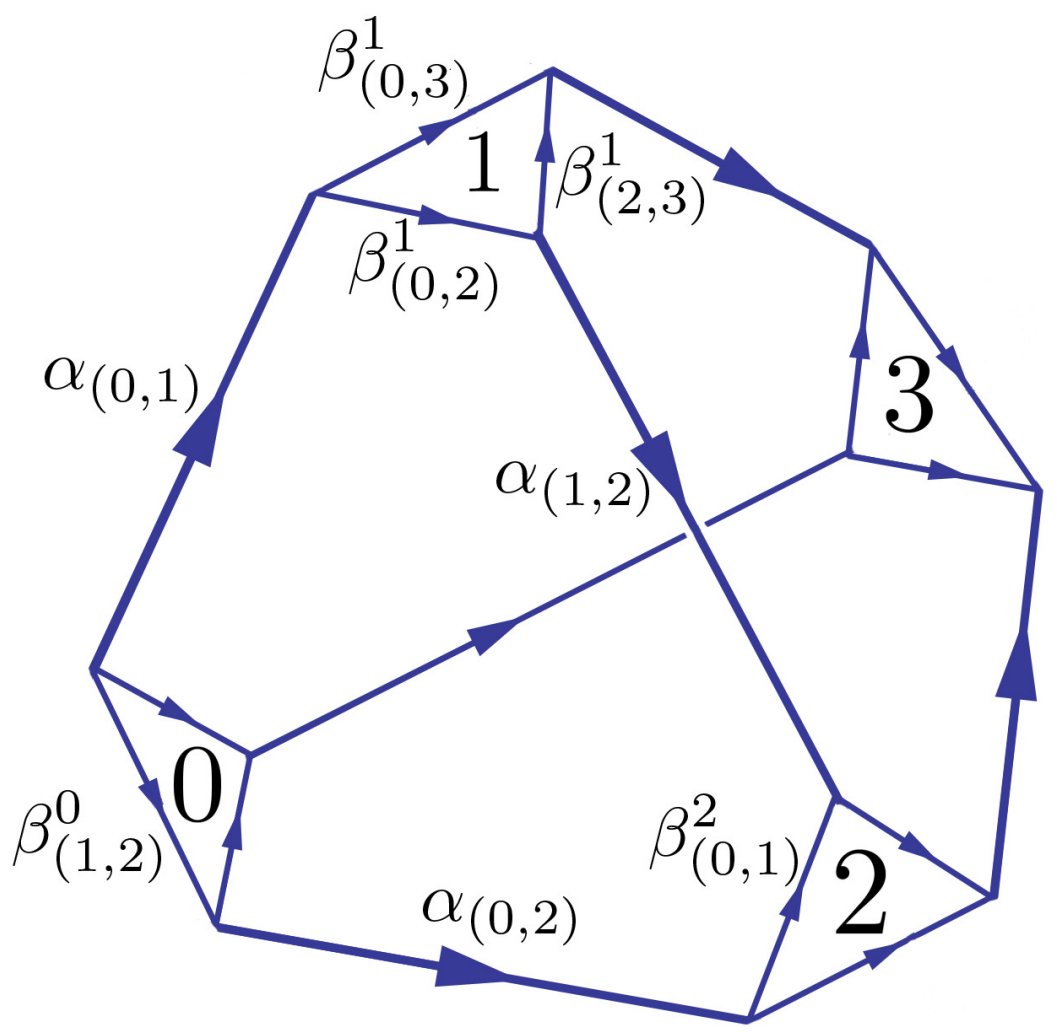

Figure 8: Labeled Cocycle

$\alpha_{(0,1)} \beta_{(0,2)}^{1} \alpha_{(1,2)}\left(\beta_{(0,1)}^{2}\right)^{-1}\left(\alpha_{(0,2)}\right)^{-1}\left(\beta_{(1,2)}^{0}\right)^{-1}=I$ and $\beta_{(0,2)}^{1} \beta_{(2,3)}^{1}\left(\beta_{(0,3)}^{1}\right)^{-1}=I$.

Such a $(G, P)$ cocycle naturally defines a boundary parabolic representation: given an element $\lambda$ of $\pi_{1}(M)$, homotope it to a path that lies on the edges of the triangulation and then represent the $\lambda$ as the product (respecting orientation) of the decoration matrices. The representation is well defined since the product around each face is the identity. Call this map from $(G, P)$ cocycles to representations $\mathcal{G}$. 


\subsubsection{Ptolemy Variety Parameterizes $(G, P)$ Cocycles}

In the above we have seen that points in the Ptolemy variety are decorations of the ideal ordered triangulation $\mathcal{T}$ and $(G, P)$ cocycles are decorations of the truncated ordered triangulation $\mathcal{T}_{t}$. Define a map $\mathcal{F}$ from the Ptolemy variety to the set of $(G, P)$ cocycles as shown below for a face of a tetrahedron in $\mathcal{T}$.

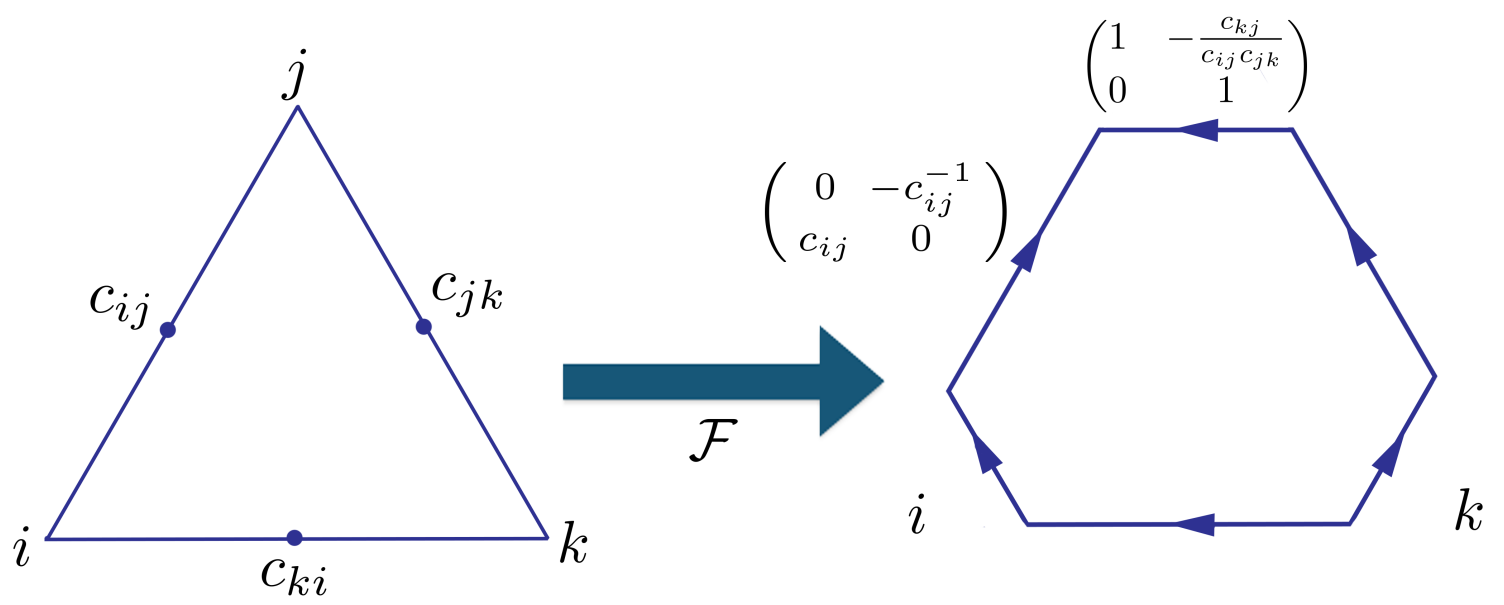

Figure 9: Ptolemy Assignment to (G,P) Cocycle

The Ptolemy relations ensure that this assignment results in a $(G, P)$ cocycle (see section 9 of [27]).

\subsubsection{Ptolemy Variety Parameterizes Boundary Parabolic Representations}

If the triangulation is sufficiently fine, the Ptolemy variety detects all $(G, P)$ representations up to conjugation via the following composition (theorem $1.3[27]$ ).

$$
\{\text { Ptolemy Assignments }\} \stackrel{\mathcal{F}}{\rightarrow}\{(G, P) \text { cocycles }\} \stackrel{\mathcal{G}}{\rightarrow}\{(G, P) \text { representations }\}
$$


In [27] barycentric cell division is proven to be sufficiently fine, though in later work Zickert and Goerner define a version of the Ptolemy variety that is triangulation independent [29]. For the following, define $\mathcal{H}:=\mathcal{G} \circ \mathcal{F}$.

\subsubsection{PSL $(2, \mathbb{C})$ Representations via Obstruction Cocycles}

The geometric representation always lifts to $\operatorname{SL}(2, \mathbb{C})$, but for a one-cusped manifold the lift is never boundary parabolic (p.3 [27]). The obstruction to lifting a boundary parabolic $\operatorname{PSL}(2, \mathbb{C})$ representation to a boundary parabolic $\mathrm{SL}(2, \mathbb{C})$ representation is a class in $H^{2}(M ; \mathbb{Z} / 2 \mathbb{Z})$ (equation $\left.9.27[27]\right)$. There is an enhanced Ptolemy variety which parameterizes boundary parabolic representations into $\operatorname{PSL}(2, \mathbb{C})$ along with an obstruction class $\sigma \in H^{2}(M ; \mathbb{Z} / 2 \mathbb{Z})$ (theorem $1.8[27]$ ). The lift to $\mathrm{SL}(2, \mathbb{C})$ of the geometric representation is detected by this enhanced Ptolemy variety. If the triangulation is sufficiently fine, the following composition is onto.

$$
\left\{\begin{array}{c}
\text { Enhanced Ptolemy } \\
\text { Assignments for } \sigma
\end{array}\right\} \rightarrow\left\{\begin{array}{c}
(G, P) \text { cocycles with } \\
\text { obstruction cocycle } \sigma
\end{array}\right\} \rightarrow\left\{\begin{array}{c}
(G, P) \text { reps with } \\
\text { obstruction cocycle } \sigma
\end{array}\right\}
$$

\subsection{VOLUME}

The next section is devoted to generalizing the notion of volume from the hyperbolic situation to boundary parabolic $\operatorname{PSL}(2, \mathbb{C})$ representations and showing how the Ptolemy variety can be used to efficiently calculate volumes. This is explained in section 9 of [27].

\subsubsection{Cheeger-Chern-Simons Invariant in the Compact Case}

As a warm up, first consider the closed manifold case. Let $\rho: \pi_{1}(M) \rightarrow S L(2, \mathbb{C})$ be a representation for a closed manifold $M$. Then, as explained in the background section 2.5.2 on geometric structures, we can build a principal $S L(2, \mathbb{C})$-bundle with flat connection

$$
E_{\rho}=\frac{\widetilde{M} \times S L(2, \mathbb{C})}{\pi_{1}(M)} \rightarrow M
$$


The action of $\pi_{1}(M)$ on $\widetilde{M} \times S L(2, \mathbb{C})$ is $\alpha \cdot(m, x)=\left(m \cdot \alpha, \rho(\alpha)^{-1} x\right)$. The connection is the pull back of the Maurer-Cartan form on $\mathrm{SL}(2, \mathbb{C})$ by the projection $p_{2}$ to $\mathrm{SL}(2, \mathbb{C})$.

Now that we have a $\mathrm{SL}(2, \mathbb{C})$ bundle associated with the representation, we can define the Cheeger-Chern-Simons invariant of $\rho$

$$
\hat{c}(\rho)=\frac{1}{2} \int_{M} s^{*}\left(\operatorname{Tr}\left(A \wedge d A+\frac{2}{3} A \wedge A \wedge A\right)\right) \in \mathbb{C} / 4 \pi^{2} \mathbb{Z}
$$

where $A$ is the flat connection of the associated $\operatorname{SL}(2, \mathbb{C})$ bundle and $s: M \rightarrow E_{\rho}$ is a section of $E_{\rho}$. Note such a section exists since $\mathrm{SL}(2, \mathbb{C})$ is 2-connected (see page 2 of [27]). This invariant is a characteristic class of flat $\operatorname{PSL}(2, \mathbb{C})$ bundles that extends our first notion of volume. Let $C S$ be the Chern-Simons invariant. Then for the geometric representation of a closed hyperbolic 3-manifold

$$
\hat{c}(\rho)=i(\operatorname{Vol}(M)+i \operatorname{CS}(M))
$$

(equation $1.2[27]$ ), motivating the following definition.

Definition 4.3.1. The complex volume of a representation is the CCS invariant $\hat{c}(\rho)$ of the associated flat $G$-bundle.

\subsubsection{CCS via Characteristic Classes}

Since CCS is a characteristic class we can choose to study the class on the universal bundle instead of our given associated bundle. For a closed manifold, a representation $\rho: \pi_{1}(M) \rightarrow$ $S L(2, \mathbb{C})$ can be upgraded to a map $f: M \rightarrow B S L(2, \mathbb{C})^{\delta}$, where $B S L(2, \mathbb{C})^{\delta}$ is the classifying space of $\operatorname{SL}(2, \mathbb{C})$ with the discrete topology. Note that $H^{3}(B S L(2, \mathbb{C}), \mathbb{C} / \mathbb{Z}) \equiv$ $\operatorname{Hom}\left(H_{3}\left(S L(2, \mathbb{C})^{\delta}\right), \mathbb{C} / \mathbb{Z}\right)$. So CCS on the universal bundle is a homomorphism

$$
\hat{c}: H_{3}(S L(2, \mathbb{C})) \rightarrow \mathbb{C} / 4 \pi^{2} \mathbb{Z}
$$




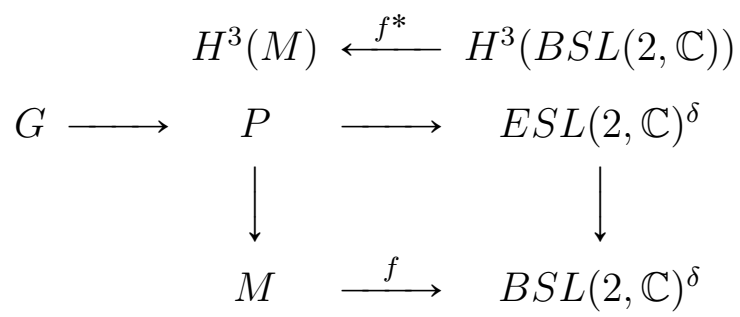

In [27], the authors first study $\hat{c}$ and then pullback by $f^{*}$ to compute the CCS of the given representation.

\subsubsection{Extend CCS to Manifolds with Boundary}

Similarly, for a manifold with boundary, a boundary parabolic representation $\rho$ gives rise to a map $B_{\rho}:(M, \partial M) \rightarrow\left(B S L(2, \mathbb{C})^{\delta}, B P\right)$. Now we can extend CCS on the universal bundle to a homomorphism $\hat{c}: H_{3}(S L(2, \mathbb{C}), P) \rightarrow \mathbb{C} / 4 \pi^{2} \mathbb{Z}$. Let $[M, \partial M]$ be a fundamental class.

Definition 4.3.2. Complex Volume (equation $1.3[27]$ ) The complex volume $\mathrm{Vol}_{\rho}$ is defined by

$$
\hat{c}\left(B_{\rho_{*}}([M, \partial M])\right)=i \operatorname{Vol}_{\mathbb{C}}(\rho)
$$

\subsubsection{Complex Volume via the Ptolemy Variety}

The main result of [27] is that the complex volume of $\operatorname{SL}(n, \mathbb{C})$ and $\operatorname{PSL}(n, \mathbb{C})$ representations

can be computed by applying an explicit function to points in the Ptolemy variety. This function factors through the extended Bloch group, which we now define (see section 3 of $[27])$.

Definition 4.3.3. The pre-Bloch group $\mathcal{P}(\mathbb{C})$ is the free abelian group on $\mathbb{C} \backslash\{0,1\}$ modulo the five term relation

$$
x-y+\frac{y}{x}-\frac{1-x^{-1}}{1-y^{-1}}+\frac{1-x}{1-y}=0, \text { for } x \neq y \in \mathbb{C} \backslash\{0,1\} .
$$

The Bloch group $\mathcal{B}(\mathbb{C})$ is the kernel of the map $\nu: \mathcal{P} \rightarrow \wedge^{2}\left(\mathbb{C}^{*}\right)$ taking $z$ to $z \wedge(1-z)$. 
Definition 4.3.4. The extended pre-Bloch group $\hat{\mathcal{P}}(\mathbb{C})$ is the free abelian group on the set

$$
\hat{C}=\left\{(e, f) \in \mathbb{C}^{2} \mid \exp (e)+\exp (f)=1\right\}
$$

modulo the lifted five term relation

$$
\left(e_{0}, f_{0}\right)-\left(e_{1}, f_{1}\right)+\left(e_{2}, f_{2}\right)-\left(e_{3}, f_{3}\right)+\left(e_{4}, f_{4}\right)=0
$$

if the equations

$$
\begin{gathered}
e_{2}=e_{1}-e_{0}, e_{3}=e_{1}-e_{0}-f_{1}+f_{0}, f_{3}=f_{2}-f_{1} \\
e_{4}=f_{0}-f_{1}, f_{4}=f_{2}-f_{1}+e_{0}
\end{gathered}
$$

are satisfied. The extended Bloch group $\hat{\mathcal{B}}(\mathbb{C})$ is the kernel of the map $\hat{\nu}: \hat{\mathcal{P}}(\mathbb{C}) \rightarrow \wedge^{2}(\mathbb{C})$ taking $(e, f)$ to $e \wedge f$.

A flattening with cross ratio $z$ is an element $(e, f) \in \hat{\mathbb{C}}$ with $\exp (e)=z$. By fixing a branch of logarithm, we can write a flattening with cross ratio $z$ as

$$
[z ; p, q]=(\log (z)+p \pi i, \log (1-z)+q \pi i)
$$

where $p, q \in \mathbb{Z}$ are even integers. For $z \in \mathbb{C}$, define Roger's dilogarithm

$$
\operatorname{Li}_{2}(z)=-\int_{0}^{z} \frac{\log (1-t)}{t} d t+\frac{1}{2} \log (z) \log (1-z)
$$

The following map, which is called the regulator, is well defined (equation 3.6 [27])

$$
\begin{gathered}
R: \hat{\mathcal{P}}(\mathbb{C}) \rightarrow \mathbb{C} / 4 \pi^{2} \mathbb{Z} \\
{[z ; p, q] \rightarrow \operatorname{Li}_{2}(z)+\frac{1}{2}(\log (z)+p \pi i)(\log (1-z)-q \pi i)-\pi^{2} / 6}
\end{gathered}
$$

For $x \in \mathbb{C} \backslash\{0\}$, use $\tilde{x}$ to denote $\log (x)$ where $\log$ is a fixed set theoretic section of the exponential map. Given a point $c$ in the Ptolemy variety (decoration of the edges in an ordered triangulation), define the map $\lambda$ as follows (equation 5.8 [27])

$$
\lambda(c)=\left(\tilde{c}_{03}+\tilde{c}_{12}-\tilde{c}_{13}, \tilde{c}_{01}+\tilde{c}_{23}-\tilde{c}_{02}-\tilde{c}_{13}\right) \in \hat{\mathcal{P}}(\mathbb{C})
$$


Then $\lambda$ has image in the extended Bloch group and the following diagram commutes (theorem $1.8[27]$ )

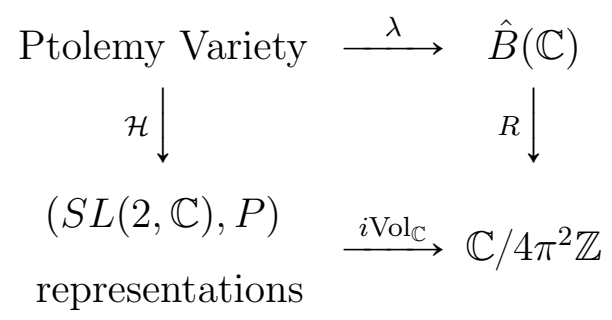

We have seen previously that the map $\mathcal{H}$ is onto if the triangulation is fine enough. In practice, points in the Ptolemy variety can be found by a computer with relative ease - the primary decomposition of the Ptolemy variety has been computed for all census manifolds with less than 9 simplices [30]. Since the maps $\lambda$ and $R$ are computationally simple, this gives an efficient way to calculate a set of complex volumes corresponding to $\mathrm{SL}(2, \mathbb{C})$ representations for a given manifold.There is an equivalent result for $P S L$ using the enhanced Ptolemy variety described in section 4.2 .5 , this is theorem 1.8 of [27]:

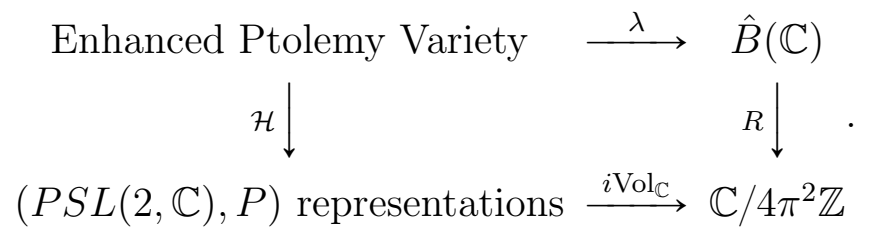

\subsection{RESULTS}

Taken in total, the above theory gives a function $F_{\text {volumes }}$ which takes in a triangulated 3manifold and outputs a set of complex volumes of boundary parabolic representations. M. Goerner implemented $F_{\text {volumes }}$ as a SnapPy module. SnapPy is a program for studying 3manifolds with torus boundary. It is a Python extension of the program SnapPea, which is written in $C$. See [20] for details about SnapPy and [30] for details about the Ptolemy module.

I implemented $F_{\text {volumes }}$ in Python, with no restriction to torus boundary manifolds, a feature that was independently implemented by M. Goerner. The goal of removing the torus 
boundary restriction was so that I could study the manifolds of the Frigerio, Martelli, and Petronio census.

\subsubsection{Frigerio, Martelli, and Petronio Census}

Frigerio, Martelli, and Petronio classified the orientable finite-volume hyperbolic 3-manifolds $M$ with non-empty and compact totally geodesic boundary and a minimum of 3 or 4 tetrahedra. Their census is available online [50] as text files of triangulations. The format of the triangulations follows the Snap Pea convention(see the Snap Pea documentation file triangulation.h for details [55]).

The lists are sorted by complexity(minimal number of tetrahedra in the triangulation), boundary genus(the boundaries are connected), and Kojima complexity (the number of tetrahedron in the canonical Kojima decomposition, see [24] for details about this decomposition).

\begin{tabular}{c|c|c|c} 
List & Complexity & Boundary Genus & Kojima Complexity \\
\hline 1 & 3 & 3 & N $/$ A \\
2 & 4 & 4 & 4 \\
3 & 4 & 3 & 4 \\
4 & 4 & 3 & 5 \\
5 & 4 & 2 & 4 \\
6 & 4 & 2 & 5 \\
7 & 4 & octahedron with dihedral angles $\pi / 6$.
\end{tabular}

I applied my implementation of $F_{\text {volumes }}$ to this census and found several interesting features. The $\mathrm{SL}(2, \mathbb{C})$ Ptolemy variety was non empty for 5 manifolds and $\operatorname{PSL}(2, \mathbb{C}) \operatorname{Ptolemy}$ variety was non empty for 49 out of the 5128 manifolds I tested. Since points in the Ptolemy variety correspond with representations, an immediate consequence is the following existence theorem.

Theorem 4.4.1. There exist non trivial boundary parabolic representations into $S L(2, \mathbb{C})$ from 3-manifolds with non torus boundary.

This result is interesting partly because there is no obvious geometric reason for such representations to exist. In particular, even though the manifolds in the Frigerio, Martelli, 
Petronio census have a geometric representation, it is never boundary parabolic since the fundamental group of the boundary is not abelian.

Once points in the Ptolemy variety are found, the software applies the maps $\lambda$ and $R$ from section 4.3.4 to obtain a complex volume, one for each equivalence class of representations detected by the Ptolemy variety. Out of the volumes, 46 have a purely imaginary complex volume and 10 have purely real complex volume. The only volumes that have nonzero real and complex part are found in list 6, which has Kojima complexity 4 and boundary genus 2. The complete list of volumes is found in appendix B.

\subsubsection{Invariant Trace Field}

The trace field of a representation $\pi_{1}(M) \rightarrow \Gamma \subset S L(2, \mathbb{C})$ is the field generated by the traces of elements of $\Gamma$. The minimal polynomial that represents the trace field of a representation that corresponds to a point in the Ptolemy variety is easily calculated (see [26]).

\subsubsection{Neumann's Conjecture}

Neumann has conjectured the Bloch group is integrally generated by hyperbolic 3-manifold elements (conjecture 1 [46]). As noted in section 4.3.4, both the Ptolemy and enhanced Ptolemy variety have image via $\lambda$ in the Bloch group. This implies that the real part of volumes calculated by $\mathcal{F}$ should be integer linear combinations of volumes of hyperbolic manifolds. That is,

Conjecture 4.4.2. (conjecture 1.16 [27]) The volume of a representation defined over a number field NF is the integral linear combination of volumes of hyperbolic manifolds with trace field contained in NF.

In [28], S. Gilles and P. Huston present numerical examples of Nuemann's conjeccture for representations of manifolds with torus boundary. First they compiled a database of 65535 manifolds including their volume and invariant trace field polynomial. Next, they examined volumes provided by the Ptolemy variety and looked for linear combinations among this database. 
I extended this numerical search into manifolds with non torus boundary. For each of the volumes I found in the Frigerio, Martelli, Petronio census, I searched for linear combinations in the Gilles, Huston database among manifolds with the same invariant trace field. I found several manifolds with complex volume $4.059 \ldots$ the same as the volume of the Dehn filled manifold $10_{1}^{2} 14(1,1)(1,1)$ up to 60 digits yielding a empirical existence result.

Theorem 4.4.3. There exist numerical examples of Neumann's conjecture 4.4.2 for volumes of representations from 3-manifolds with non torus boundary.

\subsubsection{Future Direction}

I would like to find more instances of Neumann's conjecture and explain them geometrically. It would also be interesting to characterize representations that have purely real or imaginary or zero volume.

\subsection{SOFTWARE IMPLEMENTATION}

This section outlines the software I wrote to study the complex volumes of 3-manifolds, available at github.com/samsaiki/thesis. The code that calculates complex volumes is in Python. I run the code in the SageMath [21], mathematical software that collects specialized mathematics software packages into a Python interface. By running my Python from within the SageMath environment, I have access to math specific functions such as solve which finds solutions to systems of equations.

The code which finds numerical linear combinations uses the PARI/GP computer algebra system which is designed for fast computations in number theory [53]. I also make use of Regina, a software for low dimensional topology that has no restriction to torus boundary manifolds [12]. In particular, I use the M. Goerner wrapper which imports Regina function-

ality into SnapPy so that the Ptolemy module can be applied to manifolds without torus boundary. 


\subsubsection{Complex Volumes Functions}

This subsection outlines the main functions found in the file ptolemy_variety.py, which are methods for the main class Tri which stores a triangulation of a 3-manifold. The class Tri is instantiated by a triangulation text file in Snap Pea format. The goal is to take in a text file of triangulations from the Frigerio, Martelli, Petronio census and output points in the Ptolemy variety and corresponding complex volumes.

- Edge Labeling Functions:

- NextEdge takes as input an edge and outputs the two edges that get identified via face pairings.

- LabelAllEdges uses NextEdge to label all identified edges with the same variable (reversing the sign for unordered gluings).

- Cocycle Functions:

- cocycles Returns a list of nonequivalent cocycles for the triangulation.

- $\mathrm{SL}(2, \mathbb{C})$ Ptolemy Variety Functions:

- ptolemy_equations returns a list of equations, one instance of equation 4.1 for each tetrahedron.

- $\operatorname{PSL}(2, \mathbb{C})$ Ptolemy Variety Functions:

- psl_ptolemy_equations returns a list of equations for each nonequivalent cocycle.

- Finding Points in Ptolemy Variety

- The Sage function solve takes as input a list of Ptolemy equations and returns a set of solutions if finite. If the solution set has dimension $>0$ this is indicated.

- Complex Volume Functions:

- lambda_of_triangulation implements the function $\lambda$. Input is solution to Ptolemy variety. Output is an element of the Bloch group.

- numerical_flattening Input is Bloch element. Output is flattened Bloch element.

- regulator Input is a flattened Bloch element. Output is the complex volume of that element.

- complex_volume This returns the complex volume of a point in the Ptolemy variety by applying the above three methods to each tetrahedron in the triangulation. 
The function ptolemy_equations reads a text file of 3-manifold triangulations in Snap Pea format and then applies the above functions as follows.

Input : List $L$ of triangulations of 3-Manifolds in SnapPea Format

Output: List of non zero volumes of $\operatorname{SL}(2, \mathbb{C})$ and $\operatorname{PSL}(2, \mathbb{C})$ representations

for Each triangulation $T$ in $L$ do

Check for a coherent triangulation ;

Label all identified edges of $T$ with the same variable ;

Find Ptolemy equations for $\mathrm{SL}(2, \mathbb{C})$ representations ;

For each nonequivalent cocycle find Ptolemy equations for $\operatorname{PSL}(2, \mathbb{C})$

representations ;

Apply solve to the Ptolemy equations to find points in the variety ;

Apply complex_volume to these points ;

Write nonzero volumes to a text file including the manifold triangulation ;

end

Algorithm 1: Complex Volumes of a List of Triangulations

\subsubsection{Linear Combinations}

The following functions (found in the file linear_dependence.py) takes a list of volumes and search the Gilles, Huston database (all_volumes.csv) for integer linear combinations among manifolds with the same invariant trace field.

- manifold_to_nonzero_volumes(triangulation) returns the real part of the nonzero volumes of the triangulation along with the polynomial for the associated number field.

- manifolds_with_invariant_trace_field(polynomial) opens all_volumes.csv and returns a list of manifolds whose invariant trace field is the inputted polynomial.

- is_linear_combination(volume, polynomial) uses the function manifolds_with_invariant_trace_field to generate a list $L$ of manifolds with the same invariant trace field as the inputted polynomial. Then the PARI function lindep is called to determine if there is an integral linear dependence with $L$.

- manifold_list_to_lindep(list of triangulations) 
- For each triangulation, apply manifold_to_nonzero_volumes to get a volume, polynomial pair.

- Apply is_linear_combination to the pair.

- Return a list of linear combinations. 


\subsection{PARAMETERIZING REAL PROJECTIVE STRUCTURES ON A FIXED 3-MANIFOLD}

In this chapter I introduce a method of turning ideal triangulations of a manifold into polynomial equations. Solutions to these equations correspond to projective structures and representations of the manifold.

\subsection{INTRODUCTION}

A natural problem is to represent all $(X, G)$ structures on $M$ as points in a space, which ideally is equipped with a metric. Such a space provides a framework to study how $(X, G)$ structures deform into other structures. For example, in dimension 2, Teichmüiller space parameterizes marked hyperbolic structures on a surface. As noted in section 1.3, in dimension 3 Thurston introduced the deformation variety which parameterizes hyperbolic structures on ideally triangulated 3-manifolds.

The goal of this chapter is to generalize the deformation variety to parameterize projective structures on an ideally triangulated 3 manifold. The output should be a set $P_{\text {sol }}$ of $\mathbb{R P}^{3}$ structures on the interior $M^{\circ}$ of a fixed 3-manifold $M$. Desired features roughly in order of importance are:

1. It is computationally feasible to produce the parameterization of $P_{\text {sol }}$ for simple enough $M$.

2. The holonomy representations corresponding to the geometric structures are easily recovered. 
3. $P_{\text {sol }}$ contains the unique complete hyperbolic structure on $M^{\circ}$ assuming one exists.

4. $P_{\text {sol }}$ contains some(all) properly convex structures on $M^{\circ}$.

5. Properly(or strictly) convex structures on $M^{\circ}$ are easily identified as a subvariety of $P_{\text {sol }}$.

6. $P_{\text {sol }}$ contains geometric structures which are not properly convex, for instance, the incomplete hyperbolic structures.

The remainder of this chapter presents a strategy which completely achieves 2,3 , and 4 as well as progress towards 1, 5, and 6. The main result is a partial understanding of the gluing equation variety for the figure 8 knot complement manifold, including the following.

Theorem 5.1.1. The tangent space to the gluing equation variety of the figure 8 knot complement at the complete hyperbolic structure has dimension 24.

This is complementary to other studies of the space of deformations of projective structures near the complete hyperbolic structure by, for instance, Ballas [3], [4].

\subsubsection{Strategy}

Let $\mathcal{T}$ be an abstract ideal triangulation of $M$. The strategy is to turn $\mathcal{T}$ into a geometric structure on $M$ by embedding the tetrahedron of $\mathcal{T}$ into $\mathbb{R P}^{3}$, where each vertex gets decorated with a codimension 1 hyperplane containing that point. The set of point hyperplane pairs $P F$ can be identified with signature $(1,3)$ partial flags: $P F=\{(v, f) \in$ $\left.\mathbb{R P}^{3} \times \mathbb{R P}^{3^{*}} \mid f(v)=0\right\}$. Generic tetrahedra are elements of $P F^{4}$, and up to the action of $\operatorname{PGL}(4, \mathbb{R})$, are parameterized by 5 variables I call the tetrahedral parameters. The variety $P_{\text {sol }}$ will be determined by constraining the tetrahedral and gluing parameters by face and edge holonomy equations.

\subsubsection{Motivation for Decorating with Point Hyperplane Pairs}

1. Partial flags are computationally convenient. After encoding the partial flags in homogeneous coordinates by pairs $\left\{(u, v) \in \mathbb{R}^{4} \times \mathbb{R}^{4} \mid u \cdot v=0\right\}$, the (well defined) action of $A \in \operatorname{PGL}(4, \mathbb{R})$ is simple: $A \cdot(u, v)=\left(\hat{A} \cdot u,\left(\hat{A}^{-1}\right)^{t} \cdot v\right)$ where $\hat{A} \in \operatorname{SL}(4, \mathbb{R})$ represents $A$. 
2. Point hyperplane pairs generalize the hyperbolic situation, where the supporting hyperplanes are uniquely determined as tangent to the light cone. This suggests the incomplete hyperbolic structures should be captured by $P_{\text {sol }}$.

3. In "On Convex Projective Structures and Cusps," Cooper, Long, and Tillman show all strictly convex structures have cusp ends, which preserve point and supporting hyperplane pairs [14]. Because I decorate with point hyperplane pairs, the boundary holonomy of representations of elements of $P_{\text {sol }}$ will automatically preserve a point supporting hyperplane pair.

4. The idea of decorating tetrahedra with flags has proven fruitful in parameterizing $\mathrm{SL}(3, \mathbb{C})$ structures on a hyperbolic three manifold. In "Tetrahedra of Flags, Volume, and Homology of SL(3, $\mathbb{C})$ " and a subsequent series of papers, Bergeron, Falbel, and Guilloux impose edge holonomy equations on tetrahedra decorated with flags in $\mathbb{C}^{3}$ :

$$
\left\{(x, f) \in \mathbb{C P}^{3} \times \mathbb{C P}^{3 *} \mid f(x)=0\right\}
$$

[9]. In another, parallel series of papers, Garoufalidis, Goerner, and Zickert pursue a similar approach which yields a computationally efficient method for parameterizing (boundary unipotent) representations into $\operatorname{PGL}(n, \mathbb{C})[25]$.

\subsection{PRELIMINARIES}

Let $X$ be a 3-manifold with an ordered abstract triangulation $\mathcal{T}$. We want to endow $X$ with a real projective structure. To this end we decorate the vertices of the tetrahedron of $X$ with points in $\mathbb{R P}^{3}$. Connecting a quadruple of points in $\mathbb{R P}^{3}$ by geodesics determines 8 tetrahedron, so we need more information to specify a single tetrahedron. If the points of the tetrahedron all lie on the boundary of $\mathbb{H}^{3}$, there is a natural choice of extra data: the codimension 1 hyperplane determined by the Lorentz metric (see section 5.6.1). Motivated by this natural choice we also decorate each vertex of $T \in \mathcal{T}$ with a codimension 1 hyperplane containing it, or equivalently, each vertex with a partial projective flag of signature 1,3 . This determines a projective tetrahedron since the union of two hyperplanes is separating (see 
section 5.3.2). Denote the space of such partial flags $P F$, then we call the space of quadruples of partial flags modulo the action of the isometries of $\mathbb{R P}^{3}, T P F:=P F^{4} / P G L(4, \mathbb{R})$ and refer to elements of TPF as Tetrahedrons of Partial Flags. To avoid degenerate cases, we actually include some genericity conditions on TPF to restrict to a variety of the same dimension, we call this new space $T P F^{*}$ and refer to elements as generic tetrahedron of flags. We create an analogous space of triples of flags $F P F^{*}$ which consist of Faces of Partial Flags. Having decorated we need to write down equations that determines whether the faces that are identified in $\mathcal{T}$ can be identified in $T P F^{*}$, and once identified, whether tetrahedrons glued around a single edge in $\mathcal{T}$ can also be glued in $T P F^{*}$. We call the first equations face equations and the second equations edge equations. Solutions to these equations equip $X$ with a projective structure.

We start with some preliminary definitions and notation, then in section 5.3 we describe $T P F^{*}$. In section 5.4 we define $F P F$ and write down the face equations by first defining a face parameter. In section 5.5.2 we write down the edge equations. Section 5.7 outlines software I have developed to automate the theory. Finally, in section 5.8 I apply my software to the figure-eight knot complement.

\subsubsection{Notation}

$\langle$,$\rangle is the standard inner product on \mathbb{R}^{n}$. $P(V)$ is the projectivization of the vector space

$V$. Given a point in $P\left(\mathbb{R}^{4}\right)$, we can represent it by any preimage in $\mathbb{R}^{4}$, these are called the homogeneous coordinates of $\mathbb{R P}^{n}$ and we will represent them with an over line.

\subsection{TETRAHEDRA OF PARTIAL FLAGS}

Tetrahedron of partial flags will form the geometric building blocks for the geometric structure. This section records some basic facts about such tetrahedron. 


\subsubsection{Partial flags}

A partial flag of signature $(1,3)$ is a pair $(v, f) \in P(V) \times P(V)^{*}$ such that $f(v)=0$. We call this subspace of partial flags $P F$ and refer to the first coordinate $v$ as a vertex and the second coordinate $f$ as a flag. The isomorphism $V \times V \rightarrow V \times V^{*}$ given by $(v, w) \rightarrow(v, f)$, where $f=\langle\cdot, w\rangle$ descends to $P F$, so we will think about $P F$ as pairs $(v, w) \in P(V) \times P(V)$ where $\langle w, v\rangle=0$. This isomorphism allows us to compute the action of a flag on a point in homogeneous coordinates: if $(v, f) \in P(V) \times P(V)^{*}$ then $f(v)=\langle\bar{v}, \bar{w}\rangle$ where $\bar{v}$ and $\bar{w}$ are choices of homogeneous coordinates for $v$ and $f$. For the remainder of the chapter we will perform computations in homogeneous coordinates and omit the over line where no confusion may occur.

Definition 5.3.1. For any $k$ a $k$-tuple $\left(u_{i}, v_{i}\right)$ of partial flags is called 0 -generic if $\left\langle u_{i}, v_{j}\right\rangle=0$ if and only if $i=j$ and $\left\langle v_{i}, v_{j}\right\rangle \neq 0$ for all $i, j$.

Define $T P F:=P F^{4}$ and $T P F^{*}$ to be the 0-generic subset of $T P F$.

\subsubsection{Geometric Realization of Quadruples of Flags}

Our eventual goal is to parameterize some open subset of projective structures around the complete hyperbolic structure on an ideally triangulated 3-manifold. In particular, we would like to examine deformations of the complete hyperbolic structure on the figure 8 knot complement. As we will explicitly compute in section 5.8, we can build the complete hyperbolic structure out of projective tetrahedra of flags that are 0-generic. Since being 0-generic is an open condition in $T P F$, if we deform the tetrahedra that make up the complete hyperbolic structure, they will stay 0-generic. We now turn to showing that elements of TPF determine projective tetrahedra. To streamline the argument, we include one more technical restriction on $T P F$, in essence requiring that they are properly convex.

Lemma 5.3.2. A 0-generic element $T=\left(u_{i}, v_{i}\right) \in T P F$ determines the edges of a projective tetrahedra, which we denote $E(T)$.

Proof. This lemma is based on the fact that the union of two codimension 1 projective hyperplanes is separating in $\mathbb{R} P^{3}$, although we will use the fact that $T$ is 0 -generic for a 
more explicit computation. Each pair of projective points $u_{i}, u_{j}, i \neq j$ in our tetrahedron are joined by two projective line segments. For each pair choose homogeneous coordinates $h_{i}, h_{j}$ for $v_{i}, v_{j}$ such that $\left\langle h_{i}, h_{j}\right\rangle>0$. Note this is a separate choice for each pair and is possible since $T$ is 0 -generic. Of the two projective line segments joining $u_{i}$ and $u_{j}$, we will choose the segment $S$ such that for all points $s \in S,\left\langle h_{i}, s\right\rangle\left\langle h_{j}, s\right\rangle>0$ to be the edge of our projective tetrahedron. This choice is independent of the choice of homogeneous coordinates $h_{i}$ and $h_{j}$. If $a h_{i}$ and $b h_{j}$ are different choices, then $\left\langle a h_{i}, b h_{j}\right\rangle=a b\left\langle h_{i}, h_{j}\right\rangle$ implies that $a b>0$ so that $\left\langle a h_{i}, s\right\rangle\left\langle b h_{i}, s\right\rangle$ is positive if and only if $\left\langle h_{i}, s\right\rangle\left\langle h_{j}, s\right\rangle$ is.

Definition 5.3.3. We say that $T \in T P F$ is properly convex if $E(T)$ constructed above forms a properly convex set.

Definition 5.3.4. We denote the set of all 0-generic properly convex elements of $T P F$ by $T P F^{*}$. We will call an element of $T P F^{*}$ a tetrahedron of flags.

In the following we will restrict our attention to $T P F^{*}$.

Lemma 5.3.5. A tetrahedron of flags $T \in T P F^{*}$ determines a projective tetrahedron.

Proof. Since $E(T)$ is properly convex it is contained in some affine patch. Our projective tetrahedron will be the convex hull of the tetrahedron taken in that affine patch.

\subsubsection{Action of $\operatorname{PGL}(4, \mathbb{R})$ on $P F$}

Let $A \in \operatorname{PGL}(4, \mathbb{R})$. For a representative of $A, \tilde{A} \in \mathrm{GL}(4, \mathbb{R})$, recall that for $v, w \in V$, $\langle\tilde{A} w, v\rangle=\left\langle w, \tilde{A}^{t} v\right\rangle$, so take the action of $\operatorname{PGL}(4, \mathbb{R})$ on $(u, v) \in P F$ to be

$$
A(u, v)=\left(\tilde{A} u, \tilde{A}^{-1^{t}} v\right),
$$

so that $\left(\tilde{A} u, \tilde{A}^{-1^{t}} v\right) \in P F$ since $\left\langle\tilde{A} u, \tilde{A}^{-1^{t}} v\right\rangle=\left\langle u, \tilde{A}^{t} \tilde{A}^{-1^{t}} v\right\rangle=\langle u, v\rangle=0$. This choice of

action is well defined, since if $a \tilde{A}$ is another choice of representative for $A$, and $b u, c u$ are other choices of representatives for $u$ and $v$, where $a, b, c \in \mathbb{R} \backslash\{0\}$, then

$$
\left(a \tilde{A} b u,(a \tilde{A})^{-1^{t}} c v\right)=\left(a b \tilde{A} u, \frac{c}{a} \tilde{A}^{-1^{t}} v\right) \equiv\left(\tilde{A} u, \tilde{A}^{-1^{t}} v\right)
$$

Extend the action to $P F^{4}$ diagonally. 


\subsubsection{Placing Elements of PGL $(4, \mathbb{R})$ in a Standard Position}

Lemma 5.3.6. Up to the action of $P G L(4, \mathbb{R}), 0$-generic tetrahedra of flags are parameterized by 5 real variables.

Proof. Given an ordered quadruple of partial flags $q=\left\{\left(u_{1}, v_{1}\right),\left(u_{2}, v_{2}\right),\left(u_{3}, v_{3}\right),\left(u_{4}, v_{4}\right)\right\} \in$ $T P F^{*}$, we will show that we can use an element $[A] \in P G L(4, \mathbb{R})$ to give a unique ordered representative of $[q] \in P F^{4} / P G L(4, \mathbb{R})$ of the following form:

$$
[A] \cdot[q]=\left\{\left(e_{1},\left[\begin{array}{l}
0 \\
1 \\
1 \\
x
\end{array}\right]\right),\left(e_{2},\left[\begin{array}{l}
y \\
0 \\
1 \\
1
\end{array}\right]\right),\left(e_{3},\left[\begin{array}{l}
1 \\
z \\
0 \\
1
\end{array}\right]\right),\left(e_{4},\left[\begin{array}{l}
1 \\
s \\
t \\
0
\end{array}\right]\right)\right\} .
$$

To compute a representative $A$, first note that the columns of $A^{-1}$ are determined by examining the action of $A^{-1}$ on the vertices (see equation 5.1): $A^{-1} e_{1} \equiv u_{1}, A^{-1} e_{2} \equiv$ $u_{2}, A^{-1} e_{3} \equiv u_{3}, A^{-1} e_{4} \equiv u_{4}$ implies $A^{-1}$ has columns $a u_{1}, b u_{2}, c u_{3}, d u_{4}$ where $a, b, c, d \in \mathbb{R}$. We choose to take the representative such that $c=1$. The action of flags (see equation 5.1) gives four more projective equivalences involving $a, b, d$.

$$
\left(A^{-1}\right)^{t} v_{1} \equiv\left[\begin{array}{l}
0 \\
1 \\
1 \\
x
\end{array}\right],\left(A^{-1}\right)^{t} v_{2} \equiv\left[\begin{array}{l}
y \\
0 \\
1 \\
0
\end{array}\right],\left(A^{-1}\right)^{t} v_{3} \equiv\left[\begin{array}{l}
1 \\
z \\
0 \\
1
\end{array}\right],\left(A^{-1}\right)^{t} v_{4} \equiv\left[\begin{array}{l}
1 \\
s \\
t \\
0
\end{array}\right] .
$$

Solving for $a, b$, and $d$ in the projective equivalences above gives the following representation of $A^{-1}$, where $u_{1}, u_{2}, u_{3}, u_{4}$ are column vectors.

$$
A^{-1}=\left\{\frac{\left\langle u_{3}, v_{1}\right\rangle\left\langle u_{2}, v_{3}\right\rangle}{\left\langle u_{2}, v_{1}\right\rangle\left\langle u_{1}, v_{3}\right\rangle} u_{1} \quad \frac{\left\langle u_{3}, v_{1}\right\rangle}{\left\langle u_{2}, v_{1}\right\rangle} u_{2} \quad u_{3} \quad \frac{\left\langle u_{3}, v_{2}\right\rangle}{\left\langle u_{4}, v_{2}\right\rangle} u_{4}\right\}
$$

I call this choice of $A$ the parameter matrix.

Thus tetrahedra of flags are parameterized as ordered quintuples in $\mathbb{R}$ which we call the tetrahedral parameters. 
Lemma 5.3.7. For $q=\left\{\left(u_{1}, v_{1}\right),\left(u_{2}, v_{2}\right),\left(u_{3}, v_{3}\right),\left(u_{4}, v_{4}\right)\right\} \in T P F^{*}$, the tetrahedral parameters can be calculated as

$$
\begin{gathered}
x=\frac{\left\langle u_{3}, v_{2}\right\rangle\left\langle u_{4}, v_{1}\right\rangle}{\left\langle u_{3}, v_{1}\right\rangle\left\langle u_{4}, v_{2}\right\rangle}, y=\frac{\left\langle u_{3}, v_{1}\right\rangle\left\langle u_{2}, v_{3}\right\rangle\left\langle u_{1}, v_{2}\right\rangle}{\left\langle u_{1}, v_{3}\right\rangle\left\langle u_{2}, v_{1}\right\rangle\left\langle u_{3}, v_{2}\right\rangle}, z=\frac{\left\langle u_{4}, v_{3}\right\rangle\left\langle u_{3}, v_{2}\right\rangle\left\langle u_{2}, v_{1}\right\rangle}{\left\langle u_{2}, v_{3}\right\rangle\left\langle u_{4}, v_{2}\right\rangle\left\langle u_{3}, v_{1}\right\rangle}, \\
s=\frac{\left\langle u_{1}, v_{4}\right\rangle\left\langle u_{2}, v_{3}\right\rangle}{\left\langle u_{2}, v_{3}\right\rangle\left\langle u_{2}, v_{4}\right\rangle\left\langle u_{1}, v_{3}\right\rangle}, t=\frac{\left\langle u_{2}, v_{1}\right\rangle\left\langle u_{3}, v_{4}\right\rangle}{\left\langle u_{3}, v_{1}\right\rangle\left\langle u_{2}, v_{4}\right\rangle}
\end{gathered}
$$

Proof. Apply the action of parameter matrix to the flags.

Note elements in $T P F^{*}$ are equivalence classes of tetrahedron in $\mathbb{R P}^{3}$ together with a codimension 1 hyperplanes at each vertex.

\subsection{FACES OF PARTIAL FLAGS AND FACE IDENTIFICATIONS}

Now that we have an understanding of the building blocks, we need to study how these blocks can be glued together. More precisely, in this section I calculate in terms of the tetrahedral variables when faces (triples of partial flags) of two tetrahedra can be identified by the action of $\operatorname{PGL}(4, \mathbb{R})$.

Up to the $\operatorname{PGL}(4, \mathbb{R})$-action, generic triples $F \in P F^{3}$ can be parametrized by a single variable, the face parameter. In this section we define a canonical face position and associated face parameter $f_{F}$ that allows us to check when two faces of partial flags are identified in an oriented way. We also compute the explicit elements in PGL that witness the identification. The face parameter of a decorated tetrahedron is determined by the tetrahedral parameters via a relatively simple formula. In contrast with the hyperbolic situation, the stabilizer of a generic face in $\operatorname{PGL}(4, \mathbb{R})$ with $f_{F} \neq-1$ is parameterized by a single variable, the gluing parameter $g_{F}$.

\subsubsection{Face Parameter and Standard Position}

We call a 0 -generic ordered triple a face of flags and denote the set of faces of flags as $F P F^{*}$. 
Lemma 5.4.1. Up to the action of $P G L(4, \mathbb{R})$, a face of flags is parameterized by 1 real variable.

Proof. Given such a face of flags $f=\left[\left\{\left(u_{1}, v_{1}\right),\left(u_{2}, v_{2}\right),\left(u_{3}, v_{3}\right)\right\}\right]$ we will use the action of $\operatorname{PGL}(4, \mathbb{R})$ to find a canonical representative

$$
\begin{aligned}
& \left\{\left(e_{1},\left[\begin{array}{l}
0 \\
1 \\
1 \\
0
\end{array}\right]\right),\left(e_{2},\left[\begin{array}{l}
1 \\
0 \\
1 \\
0
\end{array}\right]\right),\left(e_{3},\left[\begin{array}{l}
1 \\
f_{F} \\
0 \\
0
\end{array}\right]\right)\right\} \\
& f_{F}=\frac{\left\langle u_{1}, v_{2}\right\rangle\left\langle u_{2}, v_{3}\right\rangle\left\langle u_{3}, v_{1}\right\rangle}{\left\langle u_{1}, v_{3}\right\rangle\left\langle u_{3}, v_{2}\right\rangle\left\langle u_{2}, v_{1}\right\rangle}
\end{aligned}
$$

We refer to $f_{F}$ as the face parameter. We are trying to find an element $\left[A_{F}\right] \in P G L(4, \mathbb{R})$ that puts $F$ in standard position which is represented by a matrix $A_{F} \in G L(4, \mathbb{R})$. Just as in the proof of lemma 5.3.6, by the action on vertices, $A_{F}^{-1}$ has columns $a \cdot u_{1}, b \cdot u_{2}, c \cdot u_{3}$ and $\mathbf{r}$, where $\left\langle\mathbf{r}, v_{i}\right\rangle=0$ for $i=1,2,3$. Using the action on flags we get projective equivalences which imply $\frac{b}{c}=\frac{\left\langle u_{3}, v_{1}\right\rangle}{\left\langle u_{2}, v_{1}\right\rangle}$ and $\frac{c}{a}=\frac{\left\langle u_{1}, v_{2}\right\rangle}{\left\langle u_{3}, v_{1}\right\rangle}$. We pick $c=1$ to get a particular choice of matrix to move a face into standard position and call this matrix the face matrix for $s$. It is easy to check that for such a representative, $A_{F} s$ satisfies equation 5.6.

Lemma 5.4.2. If $f$ is a face of flags with face parameter $f_{F}$, then any even permutation of the vertices of $f$ has face parameter $f_{F}$ and any odd permutation has face parameter $1 / f_{F}$.

Proof. Direct computation using equation 5.6.

\subsubsection{Stabilizer of a Face}

Using the standard position of a face as above for a face $f$, we compute the stabilizer in $\operatorname{PGL}(4, \mathbb{R})$. If $A \cdot f=f$, then $A\left(e_{i}\right)=e_{i}$ for $i=1,2,3$, giving $A$ the form

$$
A=\left[\begin{array}{llll}
a & 0 & 0 & d \\
0 & b & 0 & e \\
0 & 0 & c & f \\
0 & 0 & 0 & g
\end{array}\right] \text { and so } A^{t}=\left[\begin{array}{cccc}
a & 0 & 0 & 0 \\
0 & b & 0 & 0 \\
0 & 0 & c & 0 \\
d & e & f & g
\end{array}\right] \text {. }
$$


Recall the action of $\operatorname{PGL}(4, \mathbb{R})$ on planes is by $A^{*}$, so stabilizing the flags means

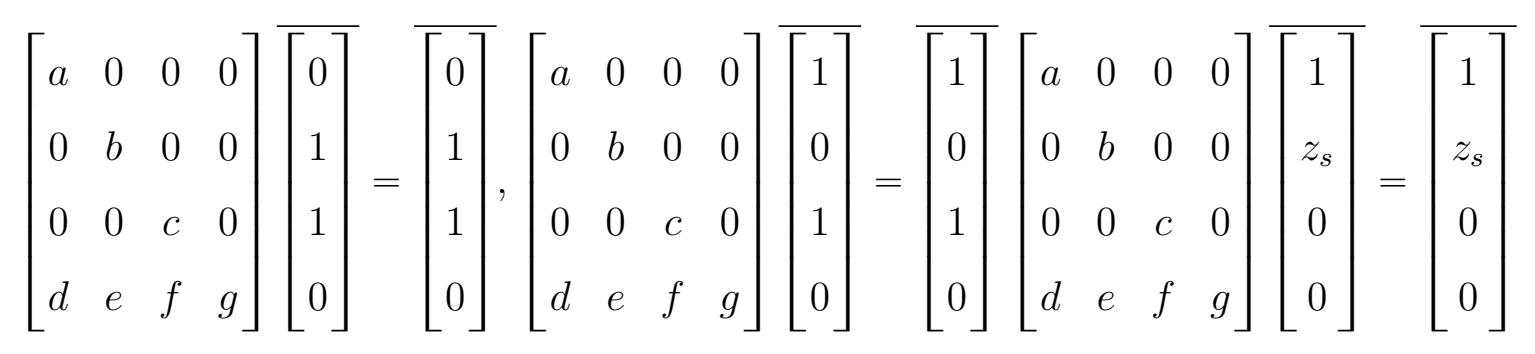

The first equations forces $b=c$, and $e=-f$. The second yields $a=c$ and $d=-f$. From the third $a=b$ and $d=-e \cdot z_{s}$. If $z=-1$, then $a=b=c$ and $d=e=-f$, and the stabilizer can be parameterized by $e$ and $g$. If $z \neq-1$, then $a=b=c$ and $d=e=f=0$, and the stabilizer can be parameterized by $g$. $A$ has a representative in $G L(4, \mathbb{R})$ :

$$
\left[\begin{array}{llll}
1 & 0 & 0 & 0 \\
0 & 1 & 0 & 0 \\
0 & 0 & 1 & 0 \\
0 & 0 & 0 & g
\end{array}\right]
$$

Motivated by the previous computations, we call a face generic if it is 0 -generic and its face parameter is not equal to -1 and make the following definition:

Definition 5.4.3. A tetrahedron of flags is generic if it is in $T P F^{*}$ and all its faces are generic. We denote the set of all generic tetrahedra of flags $G P F$.

\subsection{PROJECTIVE DEFORMATION VARIETY}

Given an oriented ideal triangulation $\mathcal{T}$ of a 3-manifold, we decorate each of the tetrahedron $T_{i} \in \mathcal{T}$ with ordered points and functionals in $\mathbb{R}^{3}$, that is, we identify each $T$ with an

element in $G P F$. If there are $n$ tetrahedra, then there are $5 n$ tetrahedral parameters which we label $x_{i}, y_{i}, z_{i}, s_{i}, t_{i}$ for $1 \leqslant i \leqslant n$. Included in the data of a ideal triangulation are $m$ face pairings $F_{i}$ for $1 \leqslant i \leqslant m$. As described in section 5.4.2, a face of flags is stabilized by a one parameter family of elements in $\operatorname{PGL}(4, \mathbb{R})$. Thus, to specify a coherent geometric structure on triangulation, for each face pairing we need to ensure that the identified faces are 
equivalent in FPF and that each time the face pairing occurs, we choose the same gluing parameter. In this section I will list equations in the tetrahedral and gluing parameters whose solutions correspond to projective structures. As discussed in section 5.5.2, the gluing parameters will be recorded by variable partial flags.

\subsubsection{Face Equations}

Lemma 5.5.1. If the faces of two tetrahedra of flags have the same face parameter, then there is an element of $P G L(4, \mathbb{R})$ that identifies the faces.

Proof. As seen in lemma 5.4.1, if the faces in two tetrahedra of flags share the same face parameter, the we can use the action of $\operatorname{PGL}(4, \mathbb{R})$ to place the face with the shared parameter in the standard position of equation 5.5.

Thus, requiring that each face identification be an isometry of faces is equivalent to equating the face parameters for both faces. This gives an equation in the tetrahedral parameters for each face pairing.

\subsubsection{Edge Equations}

For each edge in the quotient of the abstract triangulation, there is a list of face identifications that glue together a cycle of tetrahedra around this edge. Each such cycle generates a relation in the fundamental group. The edge equations will guarantee that the composition of the isometries that realize the face pairings around an edge is the identity. If we put one tetrahedron in standard position, the gluing parameter for the first face pairing determines the position of the partial flag opposite the paired face. The face equations ensure that after all the face identifications have been made, the final face is identified with the opposite face of the first tetrahedron. Pictured below is a cycle of $n$ tetrahedra looking down the common edge, which is the $1-2$ edge in $T_{1}$. Tetrahedron $T_{1}$ is in the standard position $\left\{\left(p_{1}, f_{1}\right),\left(p_{2}, f_{2}\right),\left(p_{3}, f_{3}\right),\left(p_{4}, f_{4}\right)\right\}$ as described in section 5.3.4. Introduce new variable partial flags $\left(u_{i}, v_{i}\right)$ to label the non common edge partial flags. From section 5.4.2 we see that

in terms of the tetrahedral parameters, there is a one degree of freedom for $\left(u_{1}, u_{2}\right)$ and 
one additional degree of freedom for each subsequent $\left(u_{i}, v_{i}\right)$. Also note that the gluing parameters are determined by the $\left(u_{i}, v_{i}\right)$.

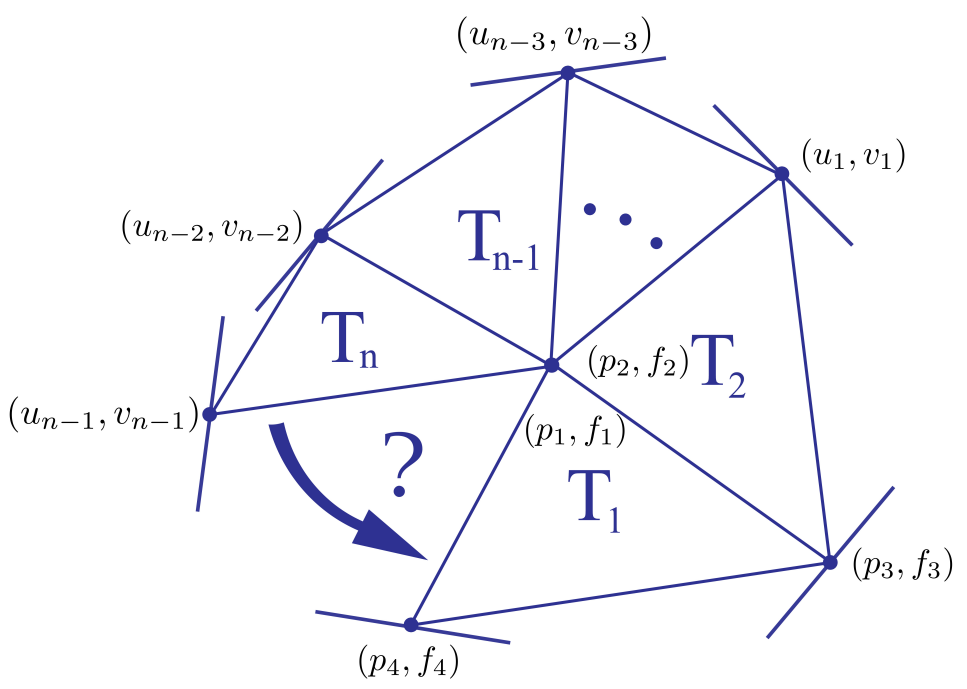

Figure 10: Edge Holonomy

There are $(n-1) \cdot 5$ edge equations, one set of five for each tetrahedron besides the one in standard position. Each of the five equations comes from calculating the tetrahedral parameters in terms of the introduced variables $\left(u_{i}, v_{i}\right)$ using equation 5.4 and setting the result equal to the original tetrahedral parameters.

\subsubsection{Gluing Equations}

As noted in the previous section, the gluing parameters can be determined from the $\left(u_{i}, v_{i}\right)$ variables. We need to introduce equations that ensure that each time a face pairing is realized in the $\left(u_{i}, v_{i}\right)$ variables it has the same gluing parameters. Choose an orientation for each of the face pairings in the abstract triangulation and label each of the two identified tetrahedron as first and second respecting orientation. For each of the occurrences of a particular face pairing $F$ in an edge holonomy, find the parameter matrix $M$ that moves the first tetrahedra $T_{1}$ into the standard position. After applying $M$ to the second tetrahedron $T_{2}$, a face of $T_{2}$ 
is completely determined in terms of the tetrahedral parameters and the vertex opposite is determined up to the gluing parameter, which will be in terms of the variables $\left(u_{i}, v_{i}\right)$ (see section 5.4.2). Equating the gluing parameter for each occurrence of the face pairing in an edge holonomy gives an additional equation in $\left(u_{i}, v_{i}\right)$ for each occurrence.

\subsection{THE HYPERBOLIC CASE}

\subsubsection{Ideal Tetrahedron of Flags}

Recall the Klein model places $\mathbb{H}^{3}$ in $\mathbb{R P}^{3}$ as the convex open subset of $[x] \in \mathbb{R} \mathbb{P}^{3}$ such that $\langle x, x\rangle_{l}<0$ and $\partial \mathbb{H}^{3}$ is the set of $[x] \in \mathbb{R P}^{3}$ such that $\langle x, x\rangle_{l}=0$ (see section C.1 for the definition of the Klein model). The tangent space to a point $x \in \partial \mathbb{H}^{3}$ is $T_{x} \mathbb{H}^{3}=$

$\left\{y \mid\langle x, y\rangle_{l}\right\}=0$. In this section we specialize the above machinery to the case of ideally triangulated hyperbolic manifolds by requiring the vertices of a tetrahedron of flags to be on the boundary of $\mathbb{H}^{3}$. In this case an arbitrary vertex has the form $[a, b, c, d]$ and the canonical choice of codimension 1 subspace is represented by $[a, b, c,-d]$, since $\langle(a, b, c, d), x\rangle_{l}=0$ if and only if $\langle(a, b, c,-d), x\rangle=0$. So ideal hyperbolic tetrahedron of flags take the form

$$
\left.\left\{\left(u_{1}, v_{1}\right),\left(u_{2}, v_{2}\right),\left(u_{3}, v_{3}\right),\left(u_{4}, v_{4}\right)\right\} \text { where } u_{i}=\left[a_{i}, b_{i}, c_{i}, d_{i}\right] \text { and } v_{i}=\left[a_{i}, b_{i}, c_{i},-d_{i}\right]\right\}
$$

\subsubsection{Coordinates for Hyperbolic Tetrahedron}

Substituting equation 5.9 into the formulas for the tetrahedral parameters 5.4, we see that hyperbolic tetrahedra of flag can be parameterized by two coordinates, say $x$ and $z$, since $s=x, t=z$, and $y=1$.

\subsubsection{Coordinates for Regular Hyperbolic Tetrahedron}

To find the coordinates of a regular hyperbolic tetrahedron, I started with a regular tetrahedron in the upper half space model $H$ which has coordinates $(0,0,0),(1,0,0),(1 / 2, \sqrt{3} / 2,0)$, 
and $\infty$. I then applied the diffeomorphisms $p: H \rightarrow J$ and $v: J \rightarrow K$ from section C.2 to obtain a regular tetrahedron in the Klein model. Finally, the action of $\operatorname{PGL}(4, \mathbb{R})$ is applied to move the tetrahedron to standard position as in section 5.3.4 and obtain the coordinates: $x=1, y=1, z=1, s=1, t=1$.

\subsubsection{Alternative Inner Product}

Unfortunately, using the standard model for $\mathbb{H}^{3}$, the canonical coordinates for a tetrahedron of flags aren't on $\delta \mathbb{H}^{3}$. The aim of this section to equip $\mathbb{R}^{4}$ with an alternative inner product $\langle\cdot, \cdot\rangle_{a}$ and repeat the construction of $H^{3} \subset \mathbb{R P}^{3}$ as $[x] \in \mathbb{R} \mathbb{P}^{3}$ such that $\langle x, x\rangle_{a}<0$.

We pull back the Lorentzian inner product by the matrix

$$
A=\left[\begin{array}{llll}
1 & 1 & 1 & 1 \\
1 & 0 & 0 & a \\
0 & 1 & 0 & b \\
0 & 0 & 1 & c
\end{array}\right]
$$

where $a^{2}+b^{2}+c^{2}=1$. Thus $<w, v>_{a}=<A w, A v>_{l}=(A v)^{t} J(A w)=v\left(A^{t} J A\right) w$. We compute

$$
A^{t} J A=\left(\begin{array}{rrrr}
0 & -1 & -1 & a-1 \\
-1 & 0 & -1 & b-1 \\
-1 & -1 & 0 & c-1 \\
a-1 & b-1 & c-1 & 0
\end{array}\right)
$$

For arbitrary $X=[x, y, z, w] \in \mathbb{R P}^{3}$, we compute

$$
F(X):=<X, X>_{a}=-2 x y-2 x z-2 y z+2(a-1) x w+2(b-1) y w+2(c-1) z w
$$

$\left.\left.D F\right|_{[} x, y, z, w\right]=2[-y-z+(a-1) w,-x-z+(b-1) w,-x-y+(c-1) w,(a-1) x+(b-1) y+(c-1) z)$.

In particular, $\left.D F\right|_{e_{1}}=-2[0,1,1,1-a],\left.D F\right|_{e_{2}}=-2[1,0,1,1-b],\left.D F\right|_{e_{3}}=-2[1,1,0,1-$ c], $\left.D F\right|_{e_{4}}=-2[1-a, 1-b, 1-c, 0]$.

By construction $A\left(e_{i}\right) \in \delta \mathbb{H}^{3}$, so in the new model for hyperbolic space $\hat{\mathbb{H}}^{3}:=A \mathbb{H}^{3}$, $e_{i} \in \delta \hat{\mathbb{H}}^{3}$. The condition that $X \in \delta \hat{\mathbb{H}}^{3}$ is that $F(X)=0$. 


\subsection{SOFTWARE IMPLEMENTATION}

In this section I will outline a group of Mathematica functions (found in the file GluingEquations.nb) that automates the process of finding the deformation equations described above. The function's input appears in parenthesis, I will abbreviate tetrahedra of flags as tetOfFlags and an abstract triangulation in SnapPea format as tri.

\subsubsection{Parameters}

- parameterMatrix(tetOfFlags) uses equation 5.3 to compute a PGL(4R) representative that moves tetOfFlags into the standard position.

- tetrahedralParameters(tetOfFlags) uses equation 5.4 to compute the five tetrahedral parameters.

- faceGlue(tetOfFlags,face uses section 5.4.1 to calculate the face matrix.

- faceParameter(tetOfFlags, face) uses equation 5.6 to compute the face parameter of the inputted face

\subsubsection{Action of $\operatorname{PGL}(4, \mathbb{R})$}

When calculating the gluing equations from section 5.5.3, it is necessary to use the action of $\operatorname{PGL}(4, \mathbb{R})$ to move an arbitrary tetrahedron of flags to the standard position. The following functions automate that process.

- actionOnFlags(matrix,flag) returns the result of the matrix acting on the partial flag (see equation 5.1).

\subsubsection{Edge Holonomy}

- edgeGluings(tri) finds the quotient edges in a SnapPea triangulation and then outputs the face gluings around each edge in the quotient of the triangulation. The complete algorithm is found in the appendix in the fundamental group section. 


\subsection{FIGURE EIGHT KNOT COMPLEMENT}

The figure-eight knot complement is a natural first choice to study. The knot complement (together with its sister knot) is the lowest volume orientable cusped hyperbolic manifold. This manifold has been extensively studied, notably by Thurston [54] who proved it had a hyperbolic structure by decomposing it into two oriented hyperbolic regular ideal tetrahedron. This decomposition appears below. Note that it is oriented, that is, the two tetrahedra are opposite each face pairing.
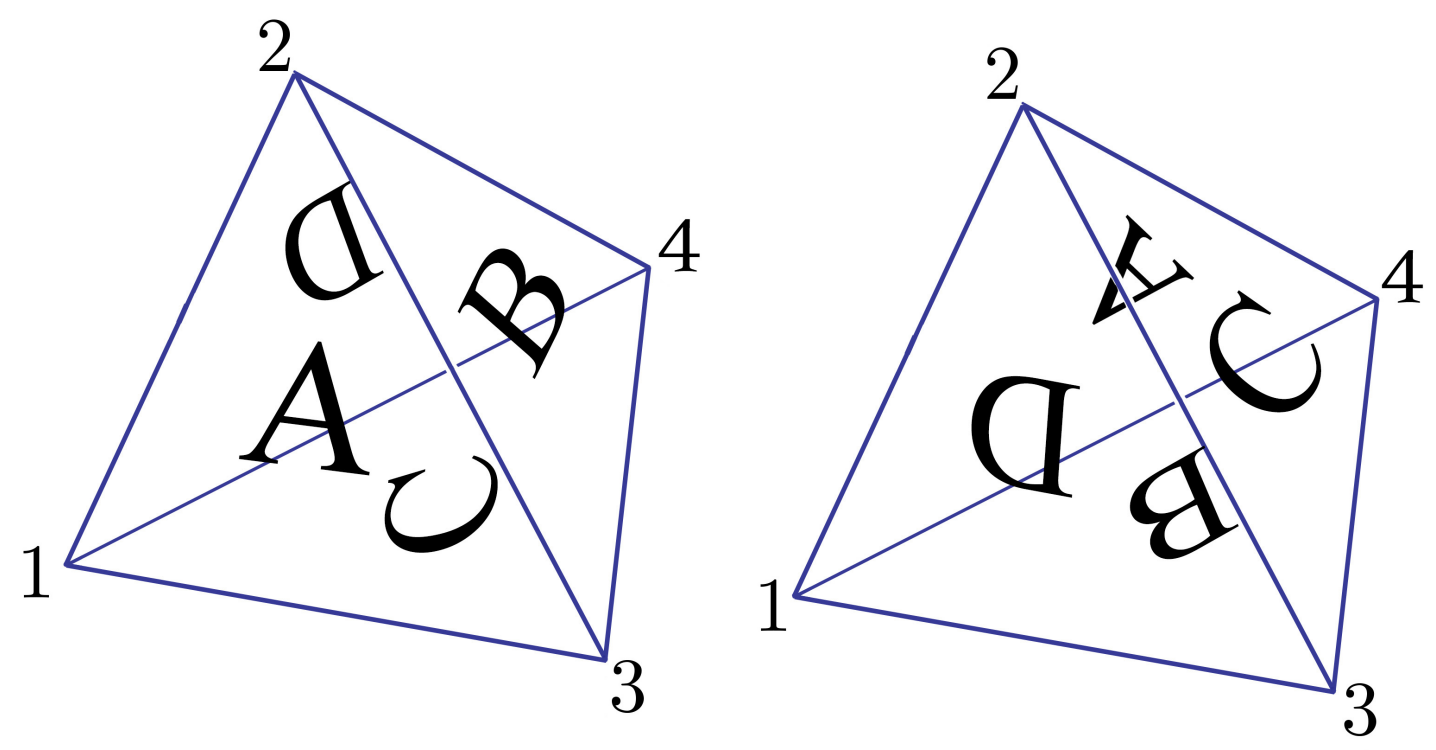

Figure 11: Triangulation of Figure 8 Knot Complement

Assign generic tetrahedron of flags to each abstract tetrahedron: $T_{1}=\left(x_{1}, y_{1}, z_{1}, s_{1}, t_{1}\right)$ and $T_{2}=\left(x_{2}, y_{2}, z_{2}, s_{2}, t_{2}\right)$. 


\subsubsection{Face Gluing Matrices}

In this section I record the face gluing matrices as calculated by my software, where $d, e, f, g$ are real parameters: Face 234 in $T_{1}$ is identified to 341 in $T_{2}$ by

$$
\left(\begin{array}{rrrr}
-\frac{e s_{1} t_{2} x_{2} z_{2}-s_{1} t_{2} x_{2} y_{1}+\left(s_{1} s_{2}-s_{1} t_{2}\right) e-s_{1}+z_{1}}{s_{1} t_{2} x_{2}+s_{1}} & 0 & 0 & 1 \\
e & 0 & 0 & 0 \\
\frac{e s_{1} t_{2} x_{2} z_{2}-s_{1} t_{2} x_{2} y_{1}-\left(s_{1} s_{2} x_{2}+s_{1}\right) e t_{2}+s_{1} t_{2} x_{2}+z_{1}}{s_{1} t_{2}^{2} x_{2}+s_{1} t_{2}} & \frac{z_{1}}{t_{2}} & 0 & 0 \\
-\frac{e s_{1} z_{2}-\left(s_{1} s_{2}-s_{1} t_{2}\right) e-s_{1} y_{1}+s_{1}-z_{1}}{s_{1} t_{2} x_{2}+s_{1}} & 0 & 1 & 0
\end{array}\right)
$$

Face 123 in $T_{1}$ is identified to 412 in $T_{2}$ by

$$
\left(\begin{array}{rrrr}
0 & 1 & 0 & -\frac{f s_{2}\left(x_{2}-1\right)+f t_{2}-x_{1}-y_{1}+1}{s_{2} x_{2} y_{2}+1} \\
0 & 0 & \frac{1}{s_{2}} & -\frac{f s_{2} t_{2} x_{2} y_{2}-s_{2} x_{1} x_{2} y_{2}-f s_{2}\left(x_{2}-1\right)+y_{1}-1}{s_{2}^{2} x_{2} y_{2}+s_{2}} \\
0 & 0 & 0 & f \\
\frac{y_{1}}{s_{2} x_{2}} & 0 & 0 & -\frac{f s_{2}^{2} x_{2} y_{2}-f s_{2} t_{2} x_{2} y_{2}+s_{2} x_{1} x_{2} y_{2}+\left(f x_{2}-x_{2} y_{2}\right) s_{2}-y_{1}}{s_{2}^{2} x_{2}^{2} y_{2}+s_{2} x_{2}}
\end{array}\right)
$$

Face 134 in $T_{1}$ is identified to 234 in $T_{2}$ by

$$
\left(\begin{array}{rrrr}
0 & d & 0 & 0 \\
\frac{1}{z_{2}} & \frac{d t_{2} y_{2} z_{2}-d\left(t_{2}+1\right) z_{2}+s_{1} s_{2}+\left(t_{2} z_{1}-t_{2}\right) z_{2}}{t_{2} z_{2}^{2}+s_{2} z_{2}} & 0 & 0 \\
0 & -\frac{d s_{2} y_{2}-d\left(s_{2}-z_{2}\right)-\left(s_{1}+1\right) s_{2}+s_{2} z_{1}}{t_{2} z_{2}+s_{2}} & 1 & 0 \\
0 & -\frac{d t_{2} y_{2} z_{2}+d\left(s_{2}-z_{2}\right)+s_{1} s_{2}-s_{2} z_{1}-t_{2} z_{2}}{t_{2} z_{2}+s_{2}} & 0 & x_{1}
\end{array}\right)
$$

Face 234 in $T_{1}$ is identified to 243 in $T_{2}$ by

$$
\left(\begin{array}{rrrr}
\frac{x_{1} y_{1}}{y_{2}} & 0 & \frac{g x_{2} y_{2} z_{2}+t_{1} x_{1} y_{1}-g y_{2}\left(z_{2}+1\right)}{y_{2}^{2} z_{2}+y_{2}} & 0 \\
0 & 1 & \frac{t_{1} x_{1} y_{1}-g x_{2}-g\left(y_{2}-1\right)}{y_{2} z_{2}+1} & 0 \\
0 & 0 & -\frac{g x_{2} y_{2} z_{2}+t_{1} x_{1} y_{1}-g\left(y_{2}-1\right)-y_{2} z_{2}-1}{y_{2} z_{2}+1} & x_{1} \\
0 & 0 & g & 0
\end{array}\right)
$$

\subsubsection{Face Pairing Equations}

$$
\left\{\frac{\mathrm{t} 1}{\mathrm{z} 1}=\frac{\mathrm{t} 2 \mathrm{x} 2}{\mathrm{~s} 2 \mathrm{z} 2}, \frac{\mathrm{t} 1 \mathrm{x} 1}{\mathrm{~s} 1 \mathrm{z} 1}=\frac{\mathrm{t} 2}{\mathrm{z} 2}, \frac{\mathrm{s} 1}{\mathrm{x} 1 \mathrm{y} 1}=\frac{\mathrm{x} 2 \mathrm{y} 2}{\mathrm{~s} 2}, \mathrm{y} 1=\frac{1}{\mathrm{y} 2}\right\}
$$




\subsubsection{Edge Holonomy Equations}

Using the function edgeGluings, we find that the abstract triangulation has 2 quotient edges. The function also identifies the 6 tetrahedron which are appear around each of the quotient edges. In this section, I will explicitly build the 6 tetrahedron that complete the edge cycle. First place tetrahedron $T_{1}$ in standard position, with vertex flag pairs $\left(p_{i}, f_{i}\right)$. The edge determines the coordinates of 2 of the 4 vertices of the remaining 5 cycle tetrahedron. Now introduce new coordinates $\left(u_{i}, v_{i}\right)$ for each of the undetermined vertices in the 5 cycle tetrahedron. The figure below is for looking down the edge 1,2 in tetrahedron $T_{1}$.

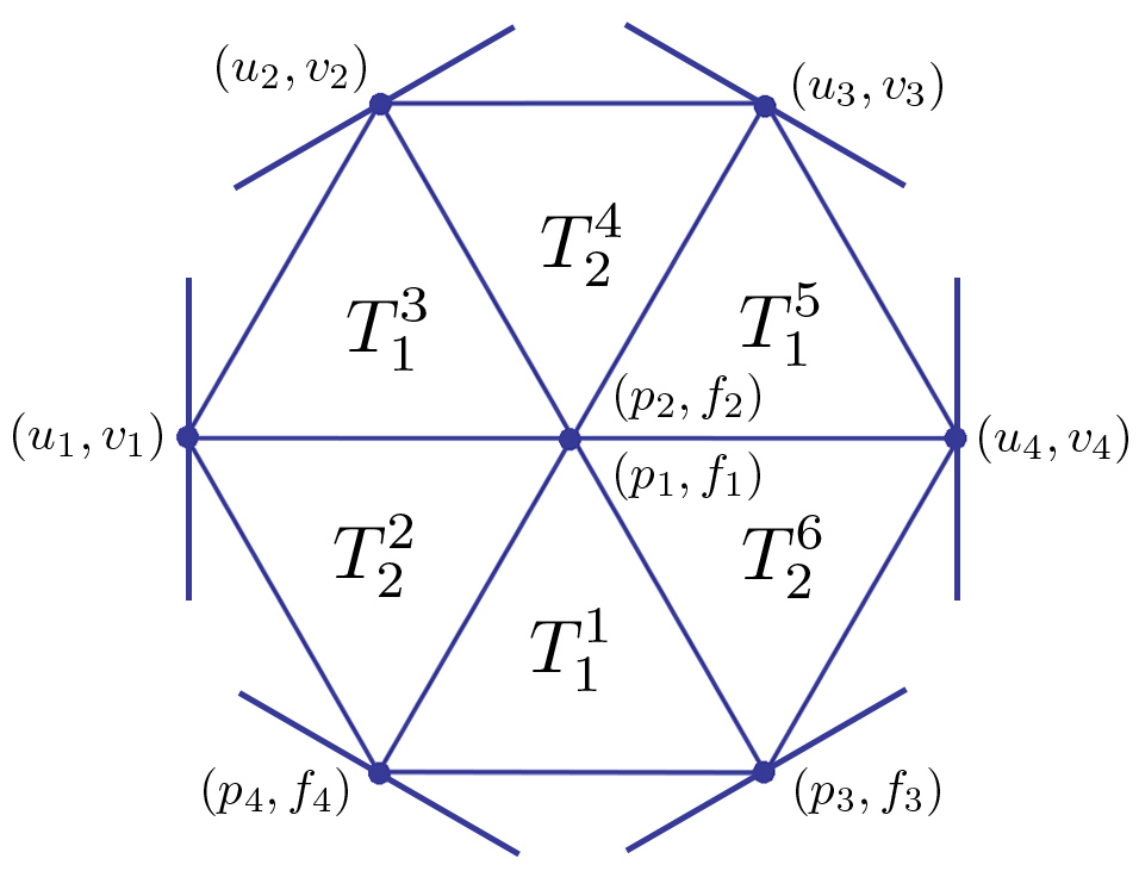

Figure 12: Variable Assignments

For each of the face identifications we compute the tetrahedral coordinates using the $\left(u_{\text {index }}, v_{\text {index }}\right)$ coordinates. This gives 5 equations for each tetrahedron in the cycle. Repeat 
the same procedure for the second edge, labeling the non edge flags as $\left(u u_{i}, v v_{i}\right)$.

$$
\begin{aligned}
& \left\{\mathrm{x} 2=\frac{\mathrm{u} 1 . \mathrm{f} 4}{\mathrm{u} 1 . \mathrm{f} 1}, \mathrm{y} 2=\frac{\mathrm{s} 1 \mathrm{v} 14 \mathrm{u} 1 . \mathrm{f} 1}{\mathrm{v} 11 \mathrm{x} 1 \mathrm{u} 1 . \mathrm{f} 4}, \mathrm{z} 2=\frac{\mathrm{v} 12 \mathrm{x} 1 \mathrm{u} 1 . \mathrm{f} 4}{\mathrm{v} 14 \mathrm{u} 1 . \mathrm{f} 1}, \mathrm{~s} 2=\frac{\mathrm{v} 14 \mathrm{y} 1}{\mathrm{v} 11}, \mathrm{t} 2=\frac{\mathrm{x} 1 \mathrm{u} 1 . \mathrm{f} 2}{\mathrm{u} 1 . \mathrm{f} 1}\right\} \\
& \left\{\mathrm{x} 1=\frac{\mathrm{y} 1 \mathrm{u} 1 . \mathrm{v} 2}{\mathrm{v} 21 \mathrm{u} 1 . \mathrm{f} 2}, \mathrm{y} 1=\frac{\mathrm{v} 21 \mathrm{u} 2 . \mathrm{f} 2}{\mathrm{v} 22 \mathrm{y} 1 \mathrm{u} 2 . \mathrm{f} 1}, \mathrm{z} 1=\frac{\mathrm{v} 22 \mathrm{y} 1 \mathrm{u} 1 . \mathrm{f} 1}{\mathrm{v} 21 \mathrm{u} 1 . \mathrm{f} 2}, \mathrm{~s} 1=\frac{\mathrm{u} 2 . \mathrm{v} 1}{\mathrm{v} 12 \mathrm{u} 2 . \mathrm{f} 1}, \mathrm{t} 1=\frac{\mathrm{v} 11 \mathrm{v} 22}{\mathrm{v} 12 \mathrm{v} 21}\right\} \\
& \left\{\mathrm{x} 2=\frac{\mathrm{u} 3 . \mathrm{f} 1 \mathrm{u} 2 . \mathrm{f} 2}{\mathrm{u} 2 . \mathrm{f} 1 \mathrm{u} 3 . \mathrm{f2}}, \mathrm{y} 2=\frac{\mathrm{v} 22 \mathrm{y} 1 \mathrm{u} 2 . \mathrm{f} 1}{\mathrm{v} 21 \mathrm{u} 2 . \mathrm{f} 2}, \mathrm{z} 2=\frac{\mathrm{u} 2 . \mathrm{f} 2 \mathrm{u} 3 . \mathrm{v} 2}{\mathrm{v} 22 \mathrm{u} 2 . \mathrm{f} 1 \mathrm{u} 3 . \mathrm{f2}}, \mathrm{s} 2=\frac{\mathrm{v} 22 \mathrm{v} 31}{\mathrm{v} 21 \mathrm{v} 32}, \mathrm{t} 2=\frac{\mathrm{u} 2 . \mathrm{v} 3}{\mathrm{v} 32 \mathrm{u} 2 . \mathrm{f} 1}\right\} \\
& \left\{\mathrm{x} 1=\frac{\mathrm{u} 4 . \mathrm{v} 3}{\mathrm{v} 32 \mathrm{u} 4 . \mathrm{f} 1}, \mathrm{y} 1=\frac{\mathrm{v} 31 \mathrm{u} 4 . \mathrm{f} 1 \mathrm{u} 3 . \mathrm{v} 4}{\mathrm{v} 41 \mathrm{u} 3 . \mathrm{f} 1 \mathrm{u} 4 . \mathrm{v} 3}, \mathrm{z} 1=\frac{\mathrm{v} 42 \mathrm{u} 3 . \mathrm{f} 1 \mathrm{u} 4 . \mathrm{v} 3}{\mathrm{v} 32 \mathrm{u} 4 . \mathrm{f} 1 \mathrm{u} 3 . \mathrm{v} 4}, \mathrm{~s} 1=\frac{\mathrm{y} 1 \mathrm{u} 3 . \mathrm{v} 4}{\mathrm{v} 41 \mathrm{u} 3 . \mathrm{f} 2}, \mathrm{t} 1=\frac{\mathrm{u} 3 . \mathrm{f} 1 \mathrm{u} 4 . \mathrm{f} 2}{\mathrm{u} 4 . \mathrm{f} 1 \mathrm{u} 3 . \mathrm{f} 2}\right\} \\
& \left\{\mathrm{x} 2=\frac{\mathrm{y} 1 \mathrm{u} 4 . \mathrm{f} 3}{\mathrm{u} 4 . \mathrm{f} 2}, \mathrm{y} 2=\frac{1}{\mathrm{y} 1}, \mathrm{z} 2=\frac{\mathrm{y} 1 \mathrm{u} 4 . \mathrm{f} 1}{\mathrm{u} 4 . \mathrm{f} 2}, \mathrm{~s} 2=\frac{\mathrm{v} 43}{\mathrm{v} 42}, \mathrm{t} 2=\frac{\mathrm{v} 41}{\mathrm{v} 42}\right\} \\
& \left\{\mathrm{x} 2=\frac{\mathrm{y} 1 \mathrm{uu} 1 . \mathrm{f} 3}{\mathrm{uu} 1 . \mathrm{f} 2}, \mathrm{y} 2=\frac{1}{\mathrm{y} 1}, \mathrm{z} 2=\frac{\mathrm{y} 1 \mathrm{uu} 1 . \mathrm{f} 1}{\mathrm{uu} 1 . \mathrm{f} 2}, \mathrm{~s} 2=\frac{\mathrm{vv} 13}{\mathrm{vv} 12}, \mathrm{t} 2=\frac{\mathrm{vv} 11}{\mathrm{vv} 12}\right\}
\end{aligned}
$$

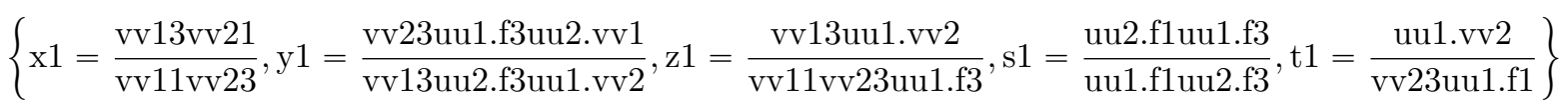

$$
\begin{aligned}
& \left\{\mathrm{x} 2=\frac{\mathrm{vv} 33 \mathrm{uu} 2 . \mathrm{f} 1}{\mathrm{uu} 2 . \mathrm{vv} 3}, \mathrm{y} 2=\frac{\mathrm{vv} 21 \mathrm{uu} 3 . \mathrm{f} 1 \mathrm{uu} 2 . \mathrm{vv} 3}{\mathrm{vv} 31 \mathrm{uu} 2 . \mathrm{f1uu} 3 . \mathrm{vv2}}, \mathrm{z} 2=\frac{\mathrm{vv} 23 \mathrm{vv} 31 \mathrm{uu} 2 . \mathrm{f} 1}{\mathrm{vv} 21 \mathrm{uu} 2 . \mathrm{vv} 3}, \mathrm{~s} 2=\frac{\mathrm{vv} 21 \mathrm{uu} 3 . \mathrm{f} 3}{\mathrm{uu} 3 . \mathrm{vv} 2}, \mathrm{t} 2=\frac{\mathrm{vv} 31 \mathrm{uu} 2 . \mathrm{f} 3}{\mathrm{uu} 2 . \mathrm{vv} 3}\right\}
\end{aligned}
$$

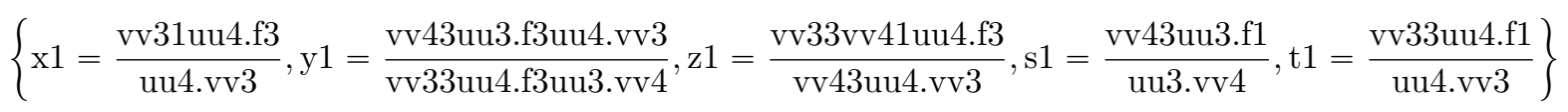

$$
\begin{aligned}
& \left\{\mathrm{x} 2=\frac{\mathrm{s} 1 \mathrm{vv} 43}{\mathrm{t} 1 \mathrm{vv} 41}, \mathrm{y} 2=\frac{\mathrm{vv} 41 \mathrm{x} 1 \mathrm{uu} 4 . \mathrm{f} 4}{\mathrm{~s} 1 \mathrm{vv} 44 \mathrm{uu} 4 . \mathrm{f} 1}, \mathrm{z} 2=\frac{\mathrm{s} 1 \mathrm{vv} 44}{\mathrm{t} 1 \mathrm{vv} 41 \mathrm{x} 1}, \mathrm{~s} 2=\frac{\mathrm{x} 1 \mathrm{uu} 4 . \mathrm{f} 3}{\mathrm{z} 1 \mathrm{uu} 4 . \mathrm{f} 1}, \mathrm{t} 2=\frac{\mathrm{vv} 44}{\mathrm{vv} 41 \mathrm{z} 1}\right\}
\end{aligned}
$$




\subsubsection{Gluing Parameter Equations}

Finally, as described in section 5.5.3, we ensure that every time a face identification takes

place, it is geometrically the same- that is, it has the same gluing parameter. So for each of the four face pairings, there are three ways of computing the gluing parameter that are equal.

$$
\begin{aligned}
& \left\{\frac{\mathrm{u} 2 . \mathrm{f} 4}{\mathrm{u} 2 . \mathrm{f1}}=\frac{\mathrm{uu} 2 . \mathrm{f} 2 \mathrm{uu} 1 . \mathrm{f} 3}{\mathrm{uu1.f2uu2.f3}}, \frac{\mathrm{uu} 2 . \mathrm{f} 2 \mathrm{uu} 1 . \mathrm{f} 3}{\mathrm{uu1.f2uu2.f3}}=\frac{\mathrm{vv} 43 \mathrm{uu} 3 . \mathrm{f} 4}{\mathrm{t} 1 \mathrm{uu} 3 . \mathrm{vv} 4}\right\}
\end{aligned}
$$

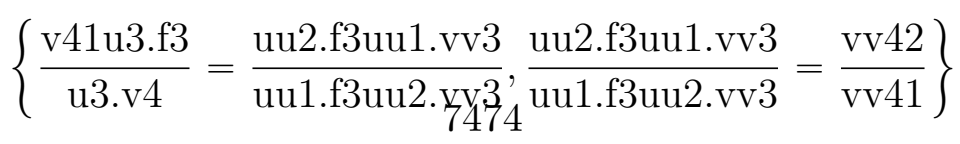

$$
\begin{aligned}
& \left\{\frac{\mathrm{v} 13}{\mathrm{v} 14}=\frac{\mathrm{v} 32 \mathrm{u} 4 . \mathrm{v} 2}{\mathrm{v} 22 \mathrm{u} 4 . \mathrm{v} 3}, \frac{\mathrm{v} 32 \mathrm{u} 4 . \mathrm{v} 2}{\mathrm{v} 22 \mathrm{u} 4 . \mathrm{v} 3}=\frac{\mathrm{uu} 4 . \mathrm{vv} 2}{\mathrm{vv} 21 \mathrm{uu} 4 . \mathrm{f} 3}\right\} \\
& \left\{\frac{\mathrm{v} 44}{\mathrm{v} 42}=\frac{\mathrm{v} 22 \mathrm{y} 1 \mathrm{u} 1 . \mathrm{v} 3}{\mathrm{v} 21 \mathrm{v} 32 \mathrm{u} 1 . \mathrm{f} 2}, \frac{\mathrm{v} 22 \mathrm{y} 1 \mathrm{u} 1 . \mathrm{v} 3}{\mathrm{v} 21 \mathrm{v} 32 \mathrm{u} 1 . \mathrm{f} 2}=\frac{\mathrm{vv} 14}{\mathrm{vv12}}\right\}
\end{aligned}
$$

\subsubsection{Finding the Complete Hyperbolic Structure}

There is exactly one complete hyperbolic structure on the figure-eight knot complement, obtained by gluing two regular tetrahedron. As seen above, all coordinates of a regular tetrahedron are 1 , so in this computation, $x_{i}=y_{i}=z_{i}=s_{i}=t_{i}=1$ for $i=1,2$. It remains to find the gluing variables. Our strategy is to first construct the geometric realization of the triangulation around both edges in the upper half space model and then move it to Klein model via the composition of the diffeomorphisms $p: H \rightarrow J$ and $t: J \rightarrow K$ found in section C.2. One tetrahedron in the triangulation around the edge is then moved to the standard position by solving for the a matrix in the appropriate equivalence class in $\operatorname{PGL}(4, \mathbb{R})$. Below, I pick the tetrahedra marked T1 to be moved to the standard position. 


\subsubsection{Zariski Tangent Space at the Complete Hyperbolic Structure}

I used Mathematica to linearize the deformation variety equations at the complete hyperbolic structure, leading to the following.

Theorem 5.8.1. The tangent space to the gluing equation variety at the complete hyperbolic structure has dimension 24.

This is complementary to results of Ballas in [3] and [4] where he explicitly finds deformations of the representation corresponding to the geometric structure on the figure 8 knot complement and then shows they correspond to deformations of projective structure. His curve of deformations have peripheral subgroups that preserve a partial flag and should be contained in the deformation variety I calculated.

\subsection{FUTURE DIRECTION}

1. By introducing more equations that restrict the boundary holonomy, I hope to identify the incomplete hyperbolic structures and the curve found by Sam Ballas in the resulting variety for the figure 8 knot complement [3].

2. If the resulting varieties are too difficult to understand completely, then I would like to find suitable subvarieties (like the incomplete hyperbolic structures) that I may be able to identify and parameterize.

3. A natural next project is analyzing the deformation variety for a simpler manifold such as the Gieseking knot complement. 
Triangulation Around Edge in $H$

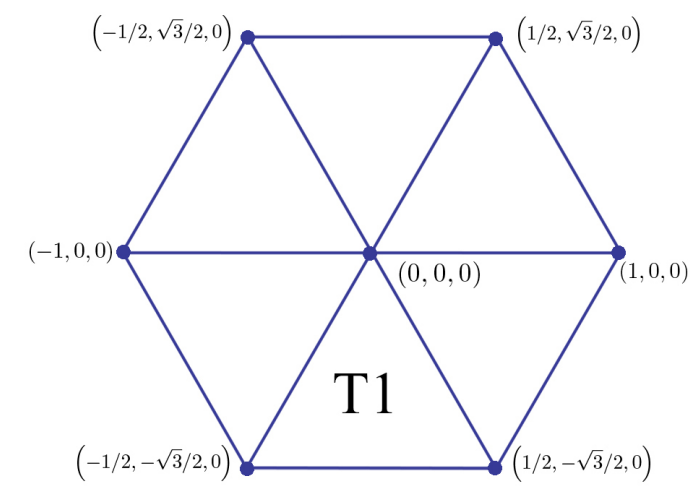

Triangulation and Flags Around Edge in $K=\mathbb{R P}^{3}$

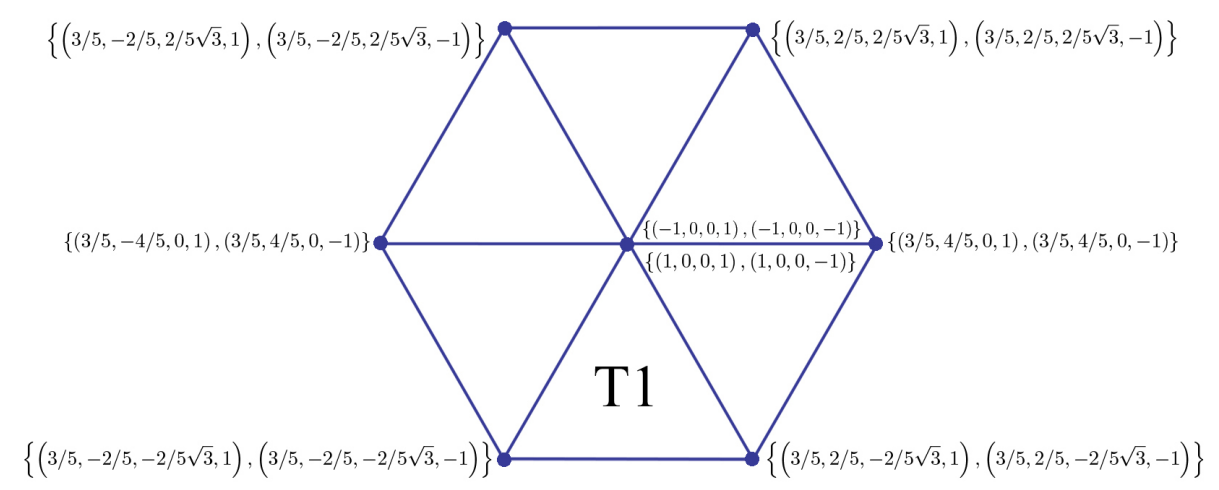

Triangulation and Flags Where T1 is in Standard Position

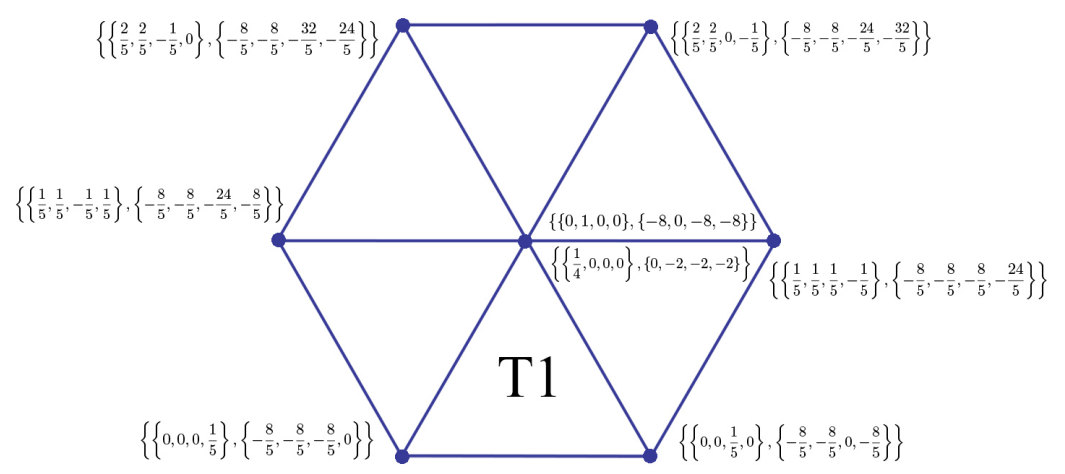

Figure 13: Triangulation Around Edge in Various Models 


\section{APPENDIX A}

\section{CODE}

Functions and algorithms will be in bold.

\section{A.1 FUNDAMENTAL GROUP CALCULATOR}

This collection of algorithms (found in the file FundamentalGroup.nb) take in a triangulated 3-manifold $T$ in SnapPea format as input and outputs a list of generators and relations for the fundamental group. The dual skeleton graph of $T$ is computed by dualSkeleton by adding a node for each tetrahedron and a directed edge for each face pairing. Generators of the fundamental group are computed as edges of a spanning tree of this graph (by the function generators. These edges, which correspond to face pairings of $T$, are labeled with letters for convenience by labeledDualSkeleton.

edgeGluings computes the relators of the fundamental group as a list of face pairings (keeping track of orientation) around each edge in the quotient of $T$ (this word of face pairings is trivial since a neighborhood of the edge is contractible). The function labelHolonomy take the list of face pairings and outputs the relators as a list of labeled (consistent with labeledDualSkeleton) generators and their inverses. 


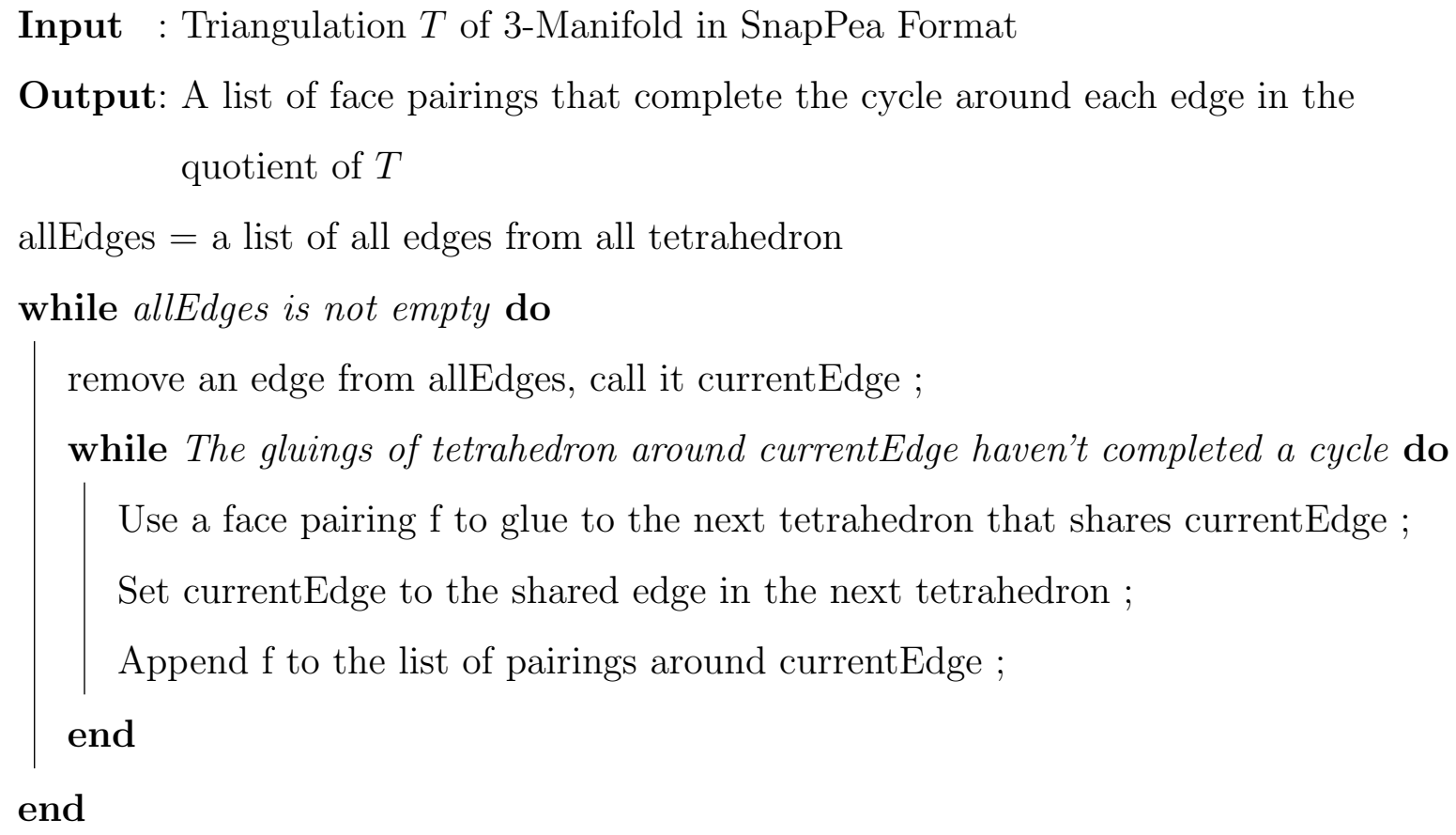

Algorithm 2: edgeGluings algorithm

\section{A.1.1 Fundamental Group of the Boundary}

The fundamental group of the boundary of the triangulation $T$ is computed in an analogous way. First manTriToBoundaryTri uses the face pairing data to output a triangulation of the boundary $T_{b}$. Then the labeled dual skeleton graph of $T_{b}$ is computed by labeledDualSkeleton2 by adding a node for each triangle in $T_{b}$ and a directed edge for each face pairing. Generators of the fundamental group are computed as edges of a spanning tree of this graph.

vertexGluings 2 computes the relators of the fundamental group as a list of face pairings (keeping track of orientation) around each vertex in the quotient of $T_{b}$ (this word of face pairings is trivial since a neighborhood of the vertex is contractible). labelBoundaryHolonomy labels these relators in a consistent manner with the above fundamental group labelings from labeledDualSkeleton. 
APPENDIX B

DATA 
Table 1: Lists 1,2,3,4,5,7

\begin{tabular}{|lc|l|}
\hline List & Tri & Complex Volume \\
\hline 1 & 1 & $0.822467033424113^{*} \mathrm{I}$ \\
1 & 2 & $0.822467033424113^{*} \mathrm{I}$ \\
1 & 3 & $\pm 0.657973626739290^{*} \mathrm{I}$ \\
1 & 4 & $\pm 0.328986813369643^{*} \mathrm{I}$ \\
\hline 2 & N/A & \\
\hline 3 & 9 & $\pm 0.328986813369649^{*} \mathrm{I}$ \\
3 & 11 & $\pm 0.657973626739286^{*} \mathrm{I}$ \\
3 & 13 & \pm 4.05976642563862 \\
3 & 17 & \pm 4.05976642563862 \\
3 & 18 & $\pm 0.657973626739296^{*} \mathrm{I}, \pm 4.05976642563862$ \\
3 & 19 & \pm 4.05976642563862 \\
\hline 4 & N/A & No representations detected \\
\hline 5 & N/A & No representations detected \\
\hline 7 & N/A & No representations detected \\
\hline
\end{tabular}


Table 2: List 6

\begin{tabular}{|c|c|c|}
\hline List Number & Tri \# & Complex Volume \\
\hline 6 & 5 & $\pm 0.328986813369647^{*} \mathrm{I}$ \\
\hline 6 & 6 & $0.657973626739293 * \mathrm{I}$ \\
\hline 6 & 8 & $0.328986813369647 * \mathrm{I}$ \\
\hline 6 & 16 & \pm 2.02988321281931 \\
\hline 6 & 17 & \pm 2.02988321281931 \\
\hline 6 & 18 & $\pm 0.657973626739288^{*} \mathrm{I}, \pm 4.05976642563862, \pm 0.657973626739291 * \mathrm{I}, \pm 0.657973626739291 * \mathrm{I}$ \\
\hline 6 & 19 & $\pm 4.05976642563862, \pm 0.657973626739291{ }^{*} \mathrm{I}$ \\
\hline 6 & 20 & $\pm 4.05976642563862, \pm 0.657973626739294^{*} \mathrm{I}$ \\
\hline 6 & 21 & $\pm 0.657973626739296 * \mathrm{I}, \pm 0.657973626739285 * \mathrm{I}, \pm 4.05976642563862, \pm 0.657973626739293 * \mathrm{I}$ \\
\hline 6 & 22 & $\pm 0.328986813369646 * \mathrm{I}$ \\
\hline 6 & 23 & $\pm 0.657973626739292 * \mathrm{I}$ \\
\hline 6 & 24 & $0,-0.428867747857092 * \mathrm{I}, \pm 2.56897060093670-0.608033159495570 * \mathrm{I}, 0,0$ \\
\hline 6 & 26 & 4.05976642563862 \\
\hline 6 & 29 & $\pm 0.657973626739285^{*} \mathrm{I}, \pm 0.657973626739296 * \mathrm{I}, \pm 0.657973626739293 * \mathrm{I}, \pm 4.05976642563862$ \\
\hline 6 & 30 & $\begin{array}{l}-0.4288677478570 * \mathrm{I}, \pm 2.568970600936-0.6080331594955 * \mathrm{I},-0.428867747857 * \mathrm{I}, \pm 2.568970600937 \\
-0.6080331594956 * \mathrm{I}\end{array}$ \\
\hline 6 & 31 & $0.469981161956636 * \mathrm{I}, 0.234990580978319 * \mathrm{I},-0.704971742934954 * \mathrm{I}$ \\
\hline 6 & 32 & - 0.234990580978318*I, 0.469981161956634*I, $0.704971742934953^{*} \mathrm{I}$ \\
\hline 6 & 34 & $0.822467033424116 * \mathrm{I}, \pm 3.66386237670888+0.822467033424113^{*} \mathrm{I}$ \\
\hline 6 & 35 & $0.822467033424116 * \mathrm{I}, \pm 3.66386237670888+0.822467033424113^{*} \mathrm{I}$ \\
\hline 6 & 38 & $\begin{array}{l}0.387983358733 * \mathrm{I}, \pm 3.770829451108-0.1939916793665 * \mathrm{I},-0.7254711937408 * \mathrm{I}, \pm 0.9427073627769- \\
0.4597314365536 * \mathrm{I}\end{array}$ \\
\hline 6 & 39 & $\begin{array}{l}0.7254711937408^{*} \mathrm{I}, \pm 0.9427073627769+0.4597314365536 * \mathrm{I},-0.6207993522147^{*} \mathrm{I}, \pm 3.166333321249 \\
-0.5120673573167 * \mathrm{I}, 0.3879833587330 * \mathrm{I}, \pm 3.770829451108+0.1939916793665 * \mathrm{I}, 0.7254711937408 * \mathrm{I}, \\
\pm 0.9427073627769+0.4597314365536 * \mathrm{I}\end{array}$ \\
\hline 6 & 40 & $0.411233516712051 * \mathrm{I}, 0.411233516712063 * \mathrm{I}, 0.411233516712063 * \mathrm{I}$ \\
\hline 6 & 41 & $0.822467033424117 * \mathrm{I}, \pm 3.66386237670888+0.822467033424113^{*} \mathrm{I}$ \\
\hline 6 & 42 & $0.822467033424121 * \mathrm{I}, \pm 3.66386237670888+0.822467033424113^{*} \mathrm{I}$ \\
\hline 6 & 43 & $0.822467033424113^{*} \mathrm{I}$ \\
\hline 6 & 48 & $\begin{array}{l} \pm 0.6985440827844-0.3324188049520^{*} \mathrm{I}, \pm 3.821687586179-0.490048228472 * \mathrm{I}, 0.5314795143743^{*} \mathrm{I} \\
\pm 2.82812208833-0.2657397571871 * \mathrm{I}\end{array}$ \\
\hline 6 & 50 & - 0.234990580978320*I, 0.469981161956635*I, 0.704971742934956*I \\
\hline 6 & 51 & $0.531479514374304^{*} \mathrm{I}, \pm 2.82812208833080-0.265739757187150 * \mathrm{I}$ \\
\hline 6 & 52 & $\pm 0.698544082784449+0.332418804952079 * \mathrm{I}, \pm 3.82168758617998+0.490048228472034 * \mathrm{I}$ \\
\hline 6 & 53 & $0.469981161956636 * \mathrm{I},-0.704971742934953 * \mathrm{I}, 0.234990580978318 * \mathrm{I}$ \\
\hline 6 & 54 & - 0.234990580978317*I, 0.704971742934955*I, - 0.469981161956637*I \\
\hline 6 & 56 & $0.822467033424117^{*} \mathrm{I}$ \\
\hline 6 & 57 & $3.66386237670888+0.822467033424113^{*} \mathrm{I}$ \\
\hline 6 & 58 & $\begin{array}{l}0.2919972076611 * \mathrm{I}, \pm 3.177293278600+0.6764684295935^{*} \mathrm{I}, 0.2919972076611 * \mathrm{I}, \pm 3.177293278600+ \\
0.6764684295935 * \mathrm{I}\end{array}$ \\
\hline 6 & 59 & Ptolemy variety is dimension 1 \\
\hline 6 & 60 & Ptolemy variety is dimension 1 \\
\hline 6 & 61 & Ptolemy variety is dimension 1 \\
\hline 6 & 62 & Ptolemy variety is dimension 1 \\
\hline 6 & 63 & $0.822467033424116^{*} \mathrm{I}$ \\
\hline 6 & 64 & $\pm 3.66386237670888+0.822467033424113^{*} \mathrm{I}$ \\
\hline
\end{tabular}




\section{APPENDIX C}

\section{ALTERNATIVE HYPERBOLIC MODELS AND ISOMETRIES}

This section contains several hyperbolic models and isometries between them that are used to find the complete hyperbolic structure on the figure-eight knot complement 5.8.

\section{C.1 THE HEMISPHERE AND KLEIN MODEL}

The hemisphere model is

$J=\left\{(w, x, y, z) \in \mathbb{R}^{4} \mid w^{2}+x^{2}+y^{2}+z^{2}=1, z>0\right\}$ with metric $d x_{J}=\frac{d w^{2}+d x^{2}+d y^{2}+d z^{2}}{d z^{2}}$

The Klein model is

$K=\left\{(w, x, y, 1) \in \mathbb{R}^{4} \mid w^{2}+x^{2}+y^{2}<1\right\}$ with $d z_{K}=\frac{d w^{2}+d y^{2}+d z^{2}}{1-d w^{2}-d x^{2}-d y^{2}}+\frac{\left(w d w^{2}+x d x^{2}+y d y^{2}\right)^{2}}{\left(1-w^{2}-x^{2}-z^{2}\right)^{2}}$

Projectivizing $K$ yields a model $P(K)$ of hyperbolic space as an open ball in $\mathbb{R} P^{3}$. 


\section{C.2 DIFFEOMORPHISMS BETWEEN MODELS}

Recall that $H$ denotes the upper half space model. Projection from $H$ to $J$ onto the point $(-1,0,0,0)$ gives the isometry $: p: H \rightarrow J, p(w, x, y, z)=(2 t-1, t x, t y, t z)$ where $t=$

$4 /\left(4+w^{2}+x^{2}+y^{2}+z^{2}\right)$. Vertical projection along the fourth coordinate gives an isometry from $J$ to $K: v: J \rightarrow K:(w, x, y, z) \mapsto(w, x, y, 1)$. 


\section{BIBLIOGRAPHY}

[1] William Abikoff. The uniformization theorem. The American Mathematical Monthly, 88(8):574-592, 1981.

[2] S. Ballas and D. D. Long. Constructing thin subgroups commensurable with the figureeight knot group. Algebraic $\mathscr{G}$ Geometric, 15:3011-3024, 2015.

[3] Samuel Ballas. Deformations of noncompact projective manifolds. Algebraic \&3 Geometric Topology, 14(5):2595-2625, 2014.

[4] Samuel Ballas. Finite volume properly convex deformations of the figure-eight knot. Geometriae Dedicata, 178(1):49-73, 2015.

[5] Samuel Ballas, Daryl Cooper, and Arielle Leitner. A classification of Generalized Cusps on Properly Convex Projective n-Manifolds. In preparation.

[6] Riccardo Benedetti and Carlo Petronio. Lectures on hyperbolic geometry. Springer Science \& Business Media, 2012.

[7] Yves Benoist. Automorphismes des cônes convexes. Inventiones mathematicae, 141(1):149-193, 2000.

[8] Yves Benoist. Convexes divisibles iii. In Annales Scientifiques de lEcole Normale Superieure, volume 38, pages 793-832. Elsevier, 2005.

[9] Nicolas Bergeron, Elisha Falbel, and Antonin Guilloux. Tetrahedra of flags, volume and homology of sl (3). Geometry \& Topology, 18(4):1911-1971, 2014.

[10] G. Besson, G. Courtois, and S. Gallot. Entropies et rigidites des espaces localement symetriques de courbure strictement negative. Geometric and Functional Analysis GAFA, 5(5):731-799, 1995.

[11] Keith Burns, Anatole Katok, W Ballman, M Brin, P Eberlein, and R Osserman. Manifolds with non-positive curvature. Ergodic Theory and Dynamical Systems, 5(02):307$317,1985$.

[12] Benjamin A. Burton, Ryan Budney, William Pettersson, et al. Regina: Software for low-dimensional topology. http://regina-normal.github.io/, 1999-2016. 
[13] James W Cannon, William J Floyd, Richard Kenyon, Walter R Parry, et al. Hyperbolic geometry. Flavors of geometry, 31:59-115, 1997.

[14] D. Cooper, D.D. Long, and S. Tillmann. On convex projective manifolds and cusps. Advances in Mathematics, 277:181 - 251, 2015.

[15] Daryl Cooper and Kelly Delp. The marked length spectrum of a projective manifold or orbifold. Proceedings of the American Mathematical Society, 138(9):3361-3376, 2010.

[16] Daryl Cooper, Darren Long, and Morwen Thistlethwaite. Computing varieties of representations of hyperbolic 3-manifolds into sl $(4, \mathbb{R})$. Experimental Mathematics, 15(3):291-305, 2006.

[17] Daryl Cooper, Darren Long, and Stephan Tillmann. Deforming convex projective manifolds. arXiv preprint arXiv:1511.06206, 2015.

[18] M. Crampon and L. Marquis. Le flot geodsique des quotients geometriquement finis des gomtries de hilbert. Pacific Journal of Mathematics, 268(2):313-369, 2014.

[19] Ch Croke, Alber Fathi, and Joel Feldman. The marked length-spectrum of a surface of nonpositive curvature. Topology, 31(4):847-855, 1992.

[20] Marc Culler, Nathan M. Dunfield, Matthias Goerner, and Jeffrey R. Weeks. SnapPy, a computer program for studying the geometry and topology of 3-manifolds. Available at http://snappy. computop.org (09/03/2017).

[21] The Sage Developers. SageMath, the Sage Mathematics Software System (Version 6.5), 2011. http://www. sagemath.org.

[22] Charles Ehresmann. Sur les espaces localement homogenes. L'Ens. Math, 35:317-333, 1936.

[23] Roberto Frigerio, Bruno Martelli, and Carlo Petronio. Small hyperbolic 3-manifolds with geodesic boundary. Experiment. Math., 13(2):171-184, 2004.

[24] Roberto Frigerio and Carlo Petronio. Construction and recognition of hyperbolic 3manifolds with geodesic boundary. Transactions of the American Mathematical Society, 356(8):3243-3282, 2004.

[25] Stavros Garoufalidis, Matthias Goerner, and Christian Zickert. Gluing equations for pgl (n, $\mathbb{C})$-representations of 3-manifolds. Algebraic \& Geometric Topology, 15(1):565-622, 2015.

[26] Stavros Garoufalidis, Matthias Goerner, and Christian Zickert. The Ptolemy field of 3-manifold representations. Algebraic \& Geometric Topology, 15(1):371-397, 2015. 
[27] Stavros Garoufalidis, Dylan P Thurston, Christian K Zickert, et al. The complex volume of sl(n,C)-representations of 3-manifolds. Duke Mathematical Journal, 164(11):2099$2160,2015$.

[28] S. Gilles and P. Huston. An experimental investigation of Neumann's conjecture. ArXiv e-prints, September 2016.

[29] M. Goerner and C. K. Zickert. Triangulation Independent Ptolemy Varieties. ArXiv e-prints, July 2015.

[30] Matthias Goerner. Ptolemy module files. http://ptolemy.unhyperbolic.org/

[31] William Goldman. Projective geometry on manifolds. Lecture notes, University of Maryland, 6, 1988.

[32] I Ya Goldsheid and Yves Guivarc'h. Zariski closure and the dimension of the gaussian low of the product of random matrices. i. Probability theory and related fields, 105(1):109$142,1996$.

[33] Allen Hatcher. Algebraic topology. 2002. Cambridge UP, Cambridge, 606(9), 2002.

[34] Jim Hoste, Morwen Thistlethwaite, and Jeff Weeks. The first 1,701,936 knots. Math. Intelligencer, 20(4):33-48, 1998.

[35] Dennis Johnson and John J. Millson. Deformation spaces associated to compact hyperbolic manifolds. Bull. Amer. Math. Soc. (N.S.), 14(1):99-102, 011986.

[36] F. W. Kamber and Ph. Tondeur. On flat bundles. Bull. Amer. Math. Soc., 72(5):846849, 091966.

[37] Michael Kapovich. Convex projective structures on gromov-thurston manifolds. Geometry $\&$ Topology, 11(3):1777-1830, 2007.

[38] D. A. Kavzdan and G. A. Margulis. A proof of Selberg's hypothesis. Mat. Sb. (N.S.), 75 (117):163-168, 1968.

[39] Felix Klein. Vergleichende betrachtungen uber neuere geometrische forschungen. Mathematische Annalen, 43(1):63-100, 1893.

[40] Jean-Louis Koszul. Deformations of locally flat connections. In Annals of the Fourier Institute, volume 18, pages 103-114, 1968.

[41] Martin W. Liebeck and Donna M. Testerman. Irreducible subgroups of algebraic groups. The Quarterly Journal of Mathematics, 55(1):47-55, 2004.

[42] Ludovic Marquis. Exemples de varietes projectives strictement convexes de volume fini en dimension quelconque. arXiv preprint arXiv:1004.3706, 2010. 
[43] Shigeyuki Morita. Geometry of characteristic classes, volume 199. American Mathematical Soc., 2001.

[44] George D Mostow. Strong rigidity of locally symmetric spaces. Number 78. Princeton University Press, 1973.

[45] Mikio Nakahara. Geometry, topology and physics. CRC Press, 2003.

[46] Walter D Neumann. Realizing arithmetic invariants of hyperbolic 3-manifolds. 2011.

[47] Bogdan Nica. Linear groups: Malcev's theorem and Selberg's lemma. arXiv preprint arXiv:1306.2385, 2013.

[48] G. Perelman. The entropy formula for the Ricci flow and its geometric applications. ArXiv Mathematics e-prints, November 2002.

[49] G. Perelman. Ricci flow with surgery on three-manifolds. ArXiv Mathematics e-prints, March 2003.

[50] Carlo Petronio. Hyperbolic 3-manifolds with non-empty boundary. http://www.dm . unipi.it/pages/petronio/publichtml/.

[51] Henri Poincare. Sur l'uniformisation des fonctions analytiques. Acta mathematica, 31(1):1-63, 1908.

[52] RB Sher and RJ Daverman. Handbook of geometric topology. Elsevier, 2001.

[53] The PARI Group, Univ. Bordeaux. PARI/GP version 2.9.0, 2016. available from http://pari.math.u-bordeaux.fr/.

[54] William P Thurston and John Willard Milnor. The geometry and topology of threemanifolds. Princeton University Princeton, 1979.

[55] Jeff Weeks. Snap pea documentation for triangulations. Available at http://www . math . uic.edu/t3m/hg/SnapPeaKernel/headers/triangulation.h. 